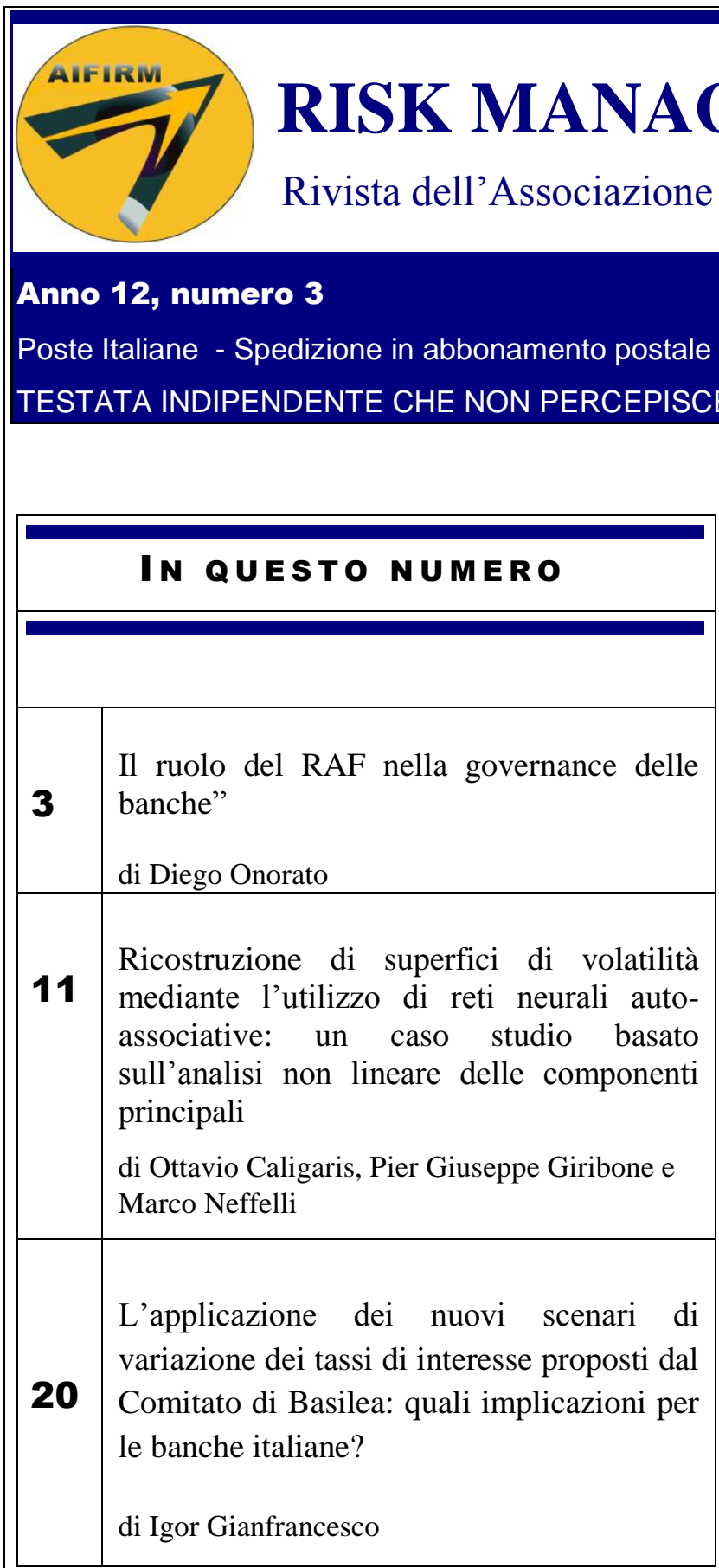

Risk Management Magazine

Anno 12, n 3 Settembre - Dicembre 2017

Direttore Responsabile:

Maurizio Vallino

Condirettore

Corrado Meglio

Consiglio scientifico

Giampaolo Gabbi (Direttore del Consiglio Scientifico), Paola Bongini, Anna Bottasso, Ruggero Bertelli, Marina Brogi, Ottavio Caligaris, Simona Cosma, Paola Ferretti, Andrea Giacomelli, Adele Grassi, Pier Giuseppe Giribone, Duccio Martelli, Laura Nieri, Pasqualina Porretta, Anna Grazia Quaranta, Enzo Scannella, Cristiana Schena, Giuseppe Torluccio.

Comitato di redazione

Emanuele Diquattro, Fausto Galmarini, Rossano Giuppa, Aldo Letizia, Paolo Palliola, Enzo Rocca, Fabio Salis

Vignettista: Silvano Gaggero

Proprietà, Redazione e Segreteria:

Associazione Italiana Financial Industry Risk

Managers (AIFIRM), Via Sile 18, 20139 Milano

Registrazione del Tribunale di Milano $n^{\circ} 629$ del $10 / 9 / 2004$

ISSN 2283-7329

E-mail: amministrazione@ aifirm.it;

Tel. 3896946315

Stampa: Algraphy S.n.c. - Passo Ponte Carrega 62-62r 16141 Genova

Le opinioni espresse negli articoli impegnano unicamente la responsabilità dei rispettivi autori

SPEDIZIONE IN ABBONAMENTO POSTALE AI SOCI AIFIRM RESIDENTI IN ITALIA, IN REGOLA CON L'ISCRIZIONE

Rivista stampata nel mese di Febbraio 2018 


\section{Processo di referaggio degli articoli proposti per la pubblicazione}

Gli articoli che sono proposti alla rivista per la pubblicazione sono sottoposti in forma anonima a due successivi livelli di referaggio.

Il primo livello di referaggio (di ammissibilità) viene effettuato sull'articolo dai membri del Consiglio Scientifico che ne valutano la congruità ai temi trattati dalla rivista.

Il secondo livello di referaggio (di pubblicabilità) viene effettuato sull'articolo da due referee scelti all'interno del Consiglio Scientifico o all'esterno tra accademici, ricercatori, esperti della materia, che ne valutano il contenuto e forma.

\section{Regolamento redazionale}

"Risk Management Magazine" è il periodico di AIFIRM (Associazione Italiana Financial Industry Risk Managers) ed interamente dedicato ai temi del risk management.

La sua struttura organizzativa prevede, oltre al direttore responsabile, un condirettore e un Consiglio Scientifico formato da accademici; quest'ultimo è garante della qualità e correttezza degli articoli pubblicati.

La rivista favorisce la diffusione di tutti i contenuti afferenti i temi del risk management, dagli aspetti normativi a quelli organizzativi e alle technicalities e vaglierà con interesse, per mezzo del Comitato Scientifico, i contributi che le perverranno.

Gli articoli proposti dovranno pervenire in formato Microsoft Word carattere Times New Roman 10 ed avere un numero di battute compreso tra 6.000 e 20.000; è gradita la presenza di tabelle e grafici.

La bibliografia deve essere redatta specificando accuratamente le fonti.

Si richiede la predisposizione di un Absract in lingua inglese.

Le opinioni espresse negli articoli impegnano unicamente la responsabilità dei rispettivi autori.

Sul sito www.aifirm.it è pubblicata la Dichiarazione sull'etica e sulle pratiche scorrette nella pubblicazione dei lavori scientifici

\section{Referee del 2017}

Si ringraziano i referee sottoelencati che hanno collaborato con la rivista nel 2017: Giampaolo Gabbi, Paola Bongini, Anna Bottasso, Ruggero Bertelli, Ottavio Caligaris, Simona Cosma, Paola Ferretti, Andrea Giacomelli, Adele Grassi, Pier Giuseppe Giribone, Duccio Martelli, Pasqualina Porretta, Anna Grazia Quaranta, Enzo Scannella, Cristiana Schena, Giuseppe Torluccio 


\title{
Il ruolo del RAF nella governance delle banche
}

\author{
di Diego Onorato (Intesa San Paolo) ${ }^{1}$
}

\section{Abstract}

Starting from 2011, regulators have intensified their attention and increased their expectations on the Risk Appetite Framework. The RAF process has therefore become a central element of the Bank governance, aimed at supporting rational growth strategies, through in-depth analysis of the risks embedded into business strategies. The position paper of AIFIRM aims at analyzing the as is situation of the Italian banking system regarding RAF implementation, with specific focus on the governance process, roles and responsibilities, integration with other governance processes, metrics used, calibration techniques, reporting approaches, risk limits cascading, escalation processes and data governance. RAF commission of AIFIRM also analyzed the answers to a survey, submitted to Italian Banks, in order to understand the different practices, and to better describe the possible approaches. Finally, in the position paper, areas of possible future development have been identified, specifically related to a further integration of the RAF in the strategic processes, other emerging risk types, strengthening of the coherence with the risk policies, consolidating of the data aggregation and reporting processes.

\section{$1 \quad$ Introduzione}

L'assunzione consapevole e la gestione dei rischi sono attività integranti del business delle banche e rappresentano aree di particolare rilevanza sia in termini di contributo alla formazione dei risultati economici che alla definizione della solidità attuale e prospettica delle banche.

A partire dal 2011, le autorità di regolamentazione hanno intensificato l'attenzione e rafforzato le aspettative sul Risk Appetite Framework come strumento di governance finalizzato ad indirizzare strategie di crescita razionali e consapevoli delle banche nell'ottica di garantire la stabilità dell'ente e, di conseguenza, del sistema finanziario.

Nel 2013 è stato pubblicato a livello europeo il "pacchetto CRDIV", volto a promuovere il rafforzamento della capacità delle banche di gestire i rischi assunti e, con specifico riferimento al contesto italiano, il 15 aggiornamento della Circ. 263/06 di Banca d'Italia ${ }^{2}$ contenente le nuove Disposizioni di Vigilanza sui controlli interni delle banche e dei gruppi bancari.

È proprio quest'ultima pubblicazione, in particolare, che ha fatto emergere l'urgenza per le banche italiane di dotarsi di un framework di risk appetite quale strumento di governance e di gestione dei rischi.

La definizione di risk appetite introdotta dal $15^{\circ}$ aggiornamento, ossia il livello di rischio - complessivo e per tipologia - che la banca intende assumere per il perseguimento dei suoi obiettivi strategici, ha posto in particolare l'attenzione su alcune tematiche centrali per il business delle banche:

- la locuzione "intende assumere" sottolinea infatti l'importanza dell'assunzione consapevole dei rischi e la valutazione del trade off "rischio/ rendimento";

- il termine "perseguimento" richiama il concetto di intenzionalità da parte delle banche nel percorso di raggiungimento dei propri obiettivi, siano essi di business e/o di rischio;

- il termine "obiettivi strategici" mette in evidenza come il concetto di risk appetite sia intimamente connesso alla formulazione delle strategie e alla definizione del modello di business attraverso cui le banche decidono di operare sul mercato.

Il panorama competitivo fortemente sfidante e in evoluzione espone le banche costantemente a fattori avversi di tipo economico, finanziario, concorrenziale e regolamentare che contribuiscono ad incrementare il livello di complessità per le funzioni incaricate di pianificare l'evoluzione del business della banca ed il relativo grado di esposizione al rischio.

Un quadro di riferimento per la determinazione della propensione al rischio completo e opportunamente articolato rappresenta anche un'opportunità per chiarire e definire un efficace assetto della governance in tema di assunzione e gestione dei rischi, nel rispetto dei dettami normativi.

A partire dal 2014, le banche italiane sono state chiamate a declinare in modo operativo le indicazioni fornite dalle nuove Disposizioni di Vigilanza, qui di seguito richiamate:

- fissare ex ante gli obiettivi di rischio/rendimento ed i conseguenti limiti operativi;

- definire i meccanismi di coerenza e raccordo tra modello di business, piano strategico, RAF, ICAAP, Budget, organizzazione aziendale, Recovery Plan e sistema dei controlli interni;

- definire, per ogni rischio materiale che la banca intende assumere, la propensione al rischio, eventuali soglie di tolleranza e limiti operativi, tenendo anche conto dei risultati delle prove di stress;

- definire le procedure e gli interventi gestionali da attivare nel caso in cui sia necessario ricondurre il livello di rischio entro l'obiettivo o i limiti prestabiliti;

\footnotetext{
${ }^{1}$ Le opinioni espresse rappresentano il punto di vista dell'autore e non riflettono necessariamente quello dell'Istituzione di appartenenza. Articolo sottoposto a doppio referaggio anonimo, pervenuto il 26/09/2017 e accettato il 20/01/2018.

${ }^{2}$ Il $15^{\circ}$ aggiornamento della circ. 263/06 di Banca d'Italia è poi confluito nella circ. 285/13.
} 
- $\quad$ definire i compiti degli organi e di tutte le funzioni aziendali coinvolte nella definizione del processo.

Nel triennio 2014-2016, le banche italiane si sono focalizzate sulla progettazione e sul consolidamento dei propri RAF individuando le più appropriate soluzioni organizzative e metodologiche: in particolare, le banche hanno dato priorità agli interventi volti a stabilire un sempre maggiore allineamento tra RAF e pianificazione strategica.

Nonostante i progressi compiuti, rimane da investigare il grado di maturità raggiunto nella definizione del Risk Appetite Framework dal sistema bancario italiano, in particolare in riferimento alle articolate sfide gestionali dell'attuale contesto competitivo.

Alla luce di queste considerazioni, la commissione RAF di AIFIRM ha predisposto un position paper, proponendosi di analizzare la situazione as is del sistema bancario italiano in tema di implementazione del RAF e di individuare, anche in funzione di benchmark con esperienze internazionali, le best practices e le principali prospettive evolutive che dovrebbero caratterizzare i prossimi anni.

Per supportare le sessioni di confronto, è stato condotto anche un sondaggio sottoposto alle Banche partecipanti alla Commissione $^{3}$, attraverso un apposito questionario di indagine collegialmente predisposto, strutturato in 4 sezioni di approfondimento:

1. "regole di governance", sezione finalizzata a comprendere il grado di integrazione del RAF con i principali processi strategici delle banche ed il ruolo e le responsabilità specifiche ricoperte dalle strutture aziendali;

2. “ambiti e metriche", sezione finalizzata a sintetizzare i principali indicatori di riferimento utilizzati a fini RAF e le metriche (appetite, tolerance, capacity, trigger, etc) oggetto di calibrazione;

3. "metodologie", sezione finalizzata ad esplorare le principali scelte metodologiche approntate dalle banche in termini di frequenza di misurazione degli indicatori e di derivazione (cascading) di limiti operativi, prove di stress, strumentazione di supporto e piani di escalation;

4. "spunti di sviluppo", sezione finalizzata a fare emergere eventuali priorità di miglioramento/approfondimento percepite dalle banche per il breve/medio termine.

Sono richiamati nel seguito del presente articolo i paragrafi sviluppati all'interno del Position Paper, riportando sinteticamente gli argomenti trattati; si rimanda al Paper stesso per una trattazione più completa in particolare in riferimento ai risultati delle analisi di benchmarking ed alle best practices individuate.

\section{Regole di governance del processo RAF}

\subsection{Articolazione del processo}

La definizione della propensione al rischio rappresenta un elemento distintivo e caratterizzante di ciascuna istituzione finanziaria e come tale risulta strettamente interconnessa alla scelta del modello di business.

I principi generali che ogni banca segue nella formalizzazione del proprio quadro di riferimento per la determinazione della propensione al rischio (RAF) ne definiscono l'identità e come tali restano sostanzialmente stabili nel medio-lungo periodo.

Non è possibile definire ex ante obiettivi di rischio/rendimento da raggiungere e conseguenti limiti operativi senza aver prima definito il business mix che si intende adottare, quindi l'eventuale presenza internazionale, l'approccio da seguire nei confronti dei diversi stakeholders, l'atteggiamento nei confronti dei competitors, le eventuali strategie di crescita e le altre variabili rilevanti.

Definito il contesto generale in cui intende operare, la banca procede a formalizzare, attraverso la definizione del RAF, obiettivi di rischio coerenti con il massimo rischio assumibile, il business model e gli indirizzi strategici. In tal senso il RAF rappresenta un elemento essenziale per la determinazione di una politica di governo dei rischi e di un processo di gestione degli stessi improntati ai principi della sana e prudente gestione aziendale.

Alla luce di tali obiettivi appare evidente l'assoluta necessità di coordinare il RAF con il processo ICAAP e di assicurarne la corretta attuazione attraverso un'organizzazione e un sistema dei controlli interni adeguati.

In estrema sintesi il RAF, tenuto conto del piano strategico e dei rischi rilevanti ivi individuati, e definito il massimo rischio assumibile, indica le tipologie di rischio che la banca intende assumere; per ciascuna tipologia di rischio, fissa gli obiettivi di rischio, le eventuali soglie di tolleranza e i limiti operativi in condizioni sia di normale operatività, sia di stress. Possono, altresì, essere indicate le circostanze, inclusi gli esiti degli scenari di stress, al ricorrere delle quali l'assunzione di determinate categorie di rischio va evitata o contenuta rispetto agli obiettivi e ai limiti fissati.

Con riferimento a questi aspetti risulta evidente la necessità di provvedere ad un aggiornamento periodico del RAF, tipicamente annuale che, tenendo conto dell'evoluzione del contesto economico e competitivo, ne garantisca la conformità ai principi generali di assunzione del rischio che la banca si è impegnata a perseguire nel medio-lungo periodo in coerenza con il business model adottato. Resta inteso che le banche devono verificare nel continuo il persistere dell'adeguatezza del framework RAF e formalizzare la possibilità di una revisione dello stesso al verificarsi di avvenimenti di rilievo, quali ad esempio variazioni eccezionali del contesto di mercato in cui operano o evoluzioni significative della configurazione societaria.

${ }^{3}$ I risultati della survey sono reperibili nel sito internet di AIFIRM. 
Le prevalenti prassi di mercato in termini di articolazione del processo di aggiornamento periodico del RAF, pur in presenza di peculiarità e ambiti di personalizzazione legati alle caratteristiche dimensionali e di complessità operativa di ciascuna banca, sono riconducibili al seguente modello di riferimento:

Principali fasi del processo RAF
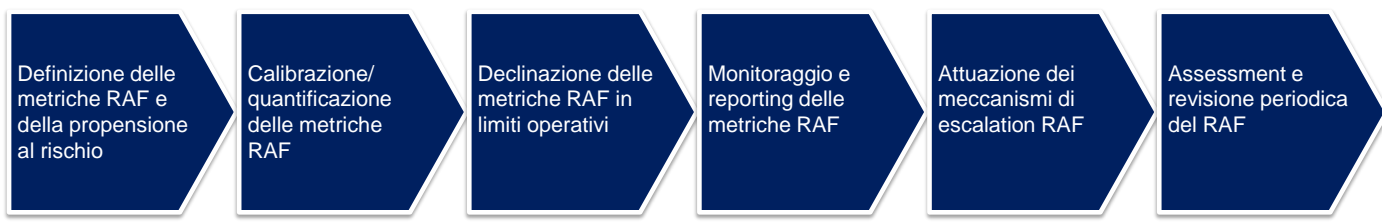

- identificazione/ aggiornamento di

- valorizzazione dei valori di risk ambiti e metriche appetite, risk RAF rispetto alle capacity e risk quali articolarela tolerance

- formulazione dei valori di limiti operativi coerent con gli obiettivi di risk appetite e di risk tolerance fissati sulle metriche RAF definite a livello complessivo

$$
\begin{array}{lll}
\text { - verifica } & \text { - attivazione di } & \text { - verifica } \\
\text { dell'andamento del } & \text { meccanismi di } & \text { dell'efficacia del } \\
\text { risk profile } & \text { escalation (piani di } & \text { RAFe della sua } \\
\text { rispetto ai valori di } & \text { emergenza e/ o } & \text { coerenza con il } \\
\text { propensione al } & \text { piani di intervento) } & \text { sistema di } \\
\text { rischio e } & & \text { governance della } \\
\text { comunicazione nei } & & \text { banca }
\end{array}
$$

comfronti degi

\subsection{Ruoli e responsabilità delle principali funzioni aziendali coinvolte}

Al fine di assicurare la corretta attuazione delle diverse fasi del processo RAF, è necessario predisporre un chiaro sistema di ruoli e di responsabilità da assegnare alle diverse funzioni e livelli della struttura organizzativa.

La presenza di un sistema di governance in grado di coinvolgere adeguatamente gli organi aziendali, i principali comitati e le diverse funzioni aziendali interessate dal processo RAF risulta infatti di fondamentale importanza per fare in modo che la consapevolezza dei rischi non sia circoscritta alle sole funzioni di risk management, ma sia adeguatamente diffusa a tutti i livelli.

A tal proposito, recentemente, il $\mathrm{SSM}^{4}$ ha sottolineato la necessità per le banche di mettere in atto idonee misure per colmare il gap, talvolta presente, tra i rischi percepiti dagli organi aziendali e quelli effettivamente assunti nell'operatività corrente.

I ruoli e le responsabilità nell'ambito del processo RAF sono frutto della combinazione, da un lato, di requisiti normativi, dall'altro di modelli di business e di governance propri delle banche.

In dettaglio vanno definite le responsabilità dell'organo con funzione di supervisione strategica, dell'organo con funzione di gestione, dell'organo con funzione di controllo, della Funzione di controllo dei rischi, e della Funzione di revisione interna.

Inoltre, particolare attenzione deve essere posta nella suddivisione delle attività operative tra le funzioni di controllo dei rischi, $\mathrm{P} \& \mathrm{C}$ e CFO e business, anche in relazione alla calibrazione delle metriche RAF.

\subsection{Integrazione del RAF con gli altri processi di governance aziendale}

Partendo dall'assunto che la gestione dei rischi costituisce uno dei principali driver per la formazione dei risultati economici delle banche, la Commissione AIFIRM concorda nel ritenere il rafforzamento della coerenza tra RAF e altri processi di governance aziendali una priorità rilevante da conseguire progressivamente nel tempo, sebbene sia già stato raggiunto un grado di integrazione complessivamente adeguato.

In particolare, il coordinamento richiesto dalle Disposizioni di Vigilanza tra RAF, Piano strategico e Budget richiede l'evoluzione dei processi di pianificazione, con conseguenti ricadute sulle metodologie di analisi e strumentazioni di supporto. I processi di pianificazione contemplano, nella prassi, unitamente alle simulazioni su profilo finanziario, economico ed organizzativo, la formulazione di previsioni prospettiche di profili di rischiosità, di adeguatezza patrimoniale e di rischio rendimento. Potrebbe sembrare quindi che gli ambiti di declinazione degli obiettivi di rischio previsti dalle Disposizioni di Vigilanza ${ }^{5}$ risultino una semplice emanazione dei processi di pianificazione.

Al contrario, la normativa RAF ha introdotto elementi di forte innovazione nelle prassi bancarie in ambito di pianificazione, portando a definire un ruolo centrale per la funzione di controllo dei rischi nell'analisi delle assunzioni di business e nella definizione di strategie di rischio, favorendo un maggiore coordinamento, anche temporale, dei processi di RAF e pianificazione annuale o pluriennale.

Oltre alle forti interrelazioni riscontrate tra il RAF ed i processi di pianificazione, un ambito nel quale il RAF risulta particolarmente impattante è quello della formulazione delle politiche retributive; infatti l'introduzione delle linee guida in tema di remuneration policy da parte dell'EBA ha consolidato un trend gestionale già in atto, teso a correlare l'effettiva erogazione delle componenti variabili della remunerazione del management al raggiungimento di target di performance risk adjusted ed, in particolare, agli indicatori utilizzati in ambito RAF.

Particolarmente rilevante risulta inoltre il tema della coerenza tra il processo RAF, l'ICAAP, il Recovery Plan ed il processo di governo delle operazioni di maggior rilievo.

${ }^{4}$ SSM, "Supervisory statement on governance and risk appetite", Giugno 2016.

${ }^{5}$ Misure espressive del capitale a rischio o capitale economico (VaR, Expected Shortfall, etc), adeguatezza patrimoniale, liquidità (Cfr Circ 285) 


\subsection{Declinazione del principio di proporzionalità}

La definizione del Risk Appetite Framework può assumere diversi livelli di complessità in funzione della dimensione dell'istituto e del suo specifico modello di business: secondo le indicazioni fornite nell'ambito della già richiamata normativa della Banca d'Italia, le banche sono chiamate ad applicare le nuove Disposizioni di Vigilanza secondo il principio di proporzionalità, "cioè tenuto conto della dimensione e complessità operative, della natura dell'attività svolta, della tipologia dei servizi prestati"

Il principio di proporzionalità risulta nella prassi declinato in diversi ambiti, quali:

- $\quad$ perimetro dei rischi coperti dal Risk Appetite Framework;

- granularità degli indicatori utilizzati;

- $\quad$ metodologie di misurazione dei rischi quantificabili;

- $\quad$ specificazione e tipologia di indicazioni qualitative per i rischi difficilmente quantificabili;

- $\quad$ articolazione degli obiettivi di rischio in limiti operativi;

- $\quad$ passaggi autorizzativi e meccanismi di escalation;

- caratteristiche della normativa interna prodotta in ambito RAF.

Per alcuni degli ambiti sopra citati, l'applicazione del principio di proporzionalità può tenere in considerazione la complessità e l'articolazione organizzativa della banca ma anche la comunicabilità e immediatezza degli indicatori (priorità agli indicatori utilizzati a livello patrimoniale e rischi di I e II pilastro), le dotazioni informatiche disponibili e la possibilità di effettivo presidio e manutenzione nel tempo.

\section{Metriche, metodologie di calibrazione e aspetti implementativi}

\subsection{Ambiti e metriche}

L'attività di mappatura dei rischi cui la banca è esposta costituisce il principale output del processo di identificazione dei fattori di potenziale vulnerabilità. In questo ambito, la definizione del set di metriche RAF è uno degli aspetti più rilevanti del processo RAF, in quanto rivela la modalità con cui le banche scelgono di sintetizzare i propri obiettivi di rischio/rendimento.

Nel definire il proprio set di metriche, le banche sono chiamate a considerare tutti i rischi materiali ed a rappresentare in modo esaustivo le principali componenti e complessità del proprio modello di business e del proprio contesto operativo. È prassi prevalente considerare, quindi, l'identificazione delle metriche RAF un'attività per le banche in continua evoluzione: da un lato, per mantenere la coerenza con le dinamiche strategiche e il grado raggiunto di diffusione della cultura del rischio, dall'altro lato per cogliere trend e scenari prospettici del contesto competitivo e nel proprio posizionamento rispetto ai rischi. Gli ambiti e la tipologia degli indicatori RAF di regola sono definiti dalle banche in coerenza a:

- Assetti e complessità organizzative (considerando come criteri, ad esempio, il dimensionamento delle reti distributive e degli organici, l'eterogeneità delle aree di business, la rilevanza delle attività cross border, etc);

- $\quad$ materialità dei rischi, rilevata anche nell'ambito di altri processi aziendali (ad esempio ICAAP);

- vulnerabilità conclamate o emergenti in seguito a prove di stress periodiche.

Un'appropriata selezione delle metriche deve supportare la migliore comprensione della natura dei rischi che i vertici aziendali intendono assumere. Pertanto la scelta degli indicatori dovrebbe considerare:

- la rilevanza strategica dell'indicatore, data l'operatività e le caratteristiche delle esposizioni o del bilancio della banca;

- $\quad$ il potenziale impatto dell'indicatore sulla gestione e sulle strategie di gestione del bilancio, evitando per esempio indicatori che possano precludere alla banca l'opportunità di aderire o sviluppare specifiche azioni sul mercato;

- l'utilizzo dell'indicatore nella comunicazione interna/esterna e nel modello di rendicontazione gestionale interna, ivi compresa l'eventuale attribuzione di responsabilità al presidio dell'indicatore in carico a specifiche funzioni interne;

- l'affidabilità della struttura di forecast/previsioni che la banca ha attivato sull'indicatore;

- la volatilità dell'indicatore e/o dipendenza dello stesso da eventi esterni non sotto il controllo della banca (es. percezione del mercato nel trend dei CDS);

- l'adeguatezza dell'indicatore rispetto a quanto utilizzato dai pari, quanto presente in letteratura e quanto richiesto dalle normative;la capacità di calibrazione: è opportuno che la scelta degli indicatori sia coerente con il set

${ }^{6} 15^{\circ}$ aggiornamento della Circ. 263/06 di Banca d'Italia, poi confluito nella circ. 285/13 
informativo e con gli strumenti e le metodologie che la banca ha a disposizione per la definizione del risk appetite, della risk tolerance e della risk capacity;

- la facilità di misurazione a consuntivo del risk profile (basi informative, frequenza, tempestività, qualità dei dati, etc) e degli eventuali scostamenti rispetto a risk appetite, tolerance e capacity;

- la capacità della banca di gestire concretamente l'indicatore attraverso azioni gestionali finalizzate alla sua correzione in caso di deviazione indesiderata dai livelli attesi o di superamento delle soglie di tolleranza.

Come indicato dalle Disposizioni di Vigilanza e confermato dal Single Supervisory Mechanism, nel RAF devono essere considerate misure quantitative e indicazioni e parametri di natura qualitativa, in modo tale da coprire tutte le aree "materiali" di rischio.

In particolare nel Position Paper sono analizzati gli indicatori ricompresi nelle seguenti classi di rischio:

1. Indicatori di adeguatezza patrimoniale e liquidità;

2. Indicatori di profitability e asset quality;

3. Altri indicatori per il monitoraggio dei singoli rischi quantificabili ovvero rischio di credito, rischio di mercato, rischio operativo, rischio tasso, leva finanziaria, rischio di concentrazione;

4. Indicatori per il monitoraggio dei rischi difficilmente quantificabili, principalmente relativi al rischio di conformità, reputazionale e di condotta.

Viene infine affrontato anche il tema della numerosità delle metriche RAF, che ancora oggi rappresenta un tema aperto e di confronto tra i diversi istituti, in relazione al principio di proporzionalità. Infatti, come emerso dal questionario effettuato, si riscontrano differenze anche significative in termini di numero degli indicatori utilizzati, da circa 10 ad oltre 50.

\subsection{Calibrazione delle metriche $R A F$}

Si richiamano le seguenti definizioni dalla normativa di Banca d'Italia:

- risk capacity (massimo rischio assumibile): il livello massimo di rischio che una banca è tecnicamente in grado di assumere senza violare i requisiti regolamentari o gli altri vincoli imposti dagli azionisti o dall'autorità di vigilanza;

- $\quad$ risk appetite (obiettivo di rischio o propensione al rischio): il livello di rischio (complessivo e per tipologia) che la banca intende assumere per il perseguimento dei suoi obiettivi strategici;

- $\quad$ risk tolerance (soglia di tolleranza): la devianza massima dal risk appetite consentita; la soglia di tolleranza è fissata in modo da assicurare in ogni caso alla banca margini sufficienti per operare, anche in condizioni di stress, entro il massimo rischio assumibile.

Un aspetto fondamentale che nel tempo è stato oggetto di progressivo consolidamento è l'approccio alla calibrazione delle metriche RAF, nella prassi differenziato tra livelli di risk capacity, appetite, tolerance, che ricomprende solitamente il riferimento a indicazioni normative, analisi di sensitivity, scenario, ipotesi judgmental, analisi di trend storici.

\subsection{Cascading dei limiti di rischio}

Le Disposizioni di Vigilanza identificano i limiti di rischio (risk limit) come "l'articolazione degli obiettivi di rischio, definiti, in linea con il principio di proporzionalità, per tipologie di rischio, unità e/o linee di business, linee di prodotto, tipologie di clienti", . Nelle prassi prevalenti, si parla alternativamente anche di allocazione o declinazione a "cascata" (cascading appunto) del risk appetite.

L'articolazione degli obiettivi di rischio identifica le modalità con cui le misure quantitative e le dichiarazioni qualitative di propensione al rischio definite a livello complessivo di Banca/Gruppo sono dettagliate su ulteriori dimensioni di analisi (siano esse tipologie di rischio, unità e/o linee di business, linee di prodotto, tipologie di clienti, portafogli).

È prassi consolidata considerare il cascading dei limiti di rischio come parte integrante del complessivo Risk Appetite Framework e come strumento operativo di attuazione del RAF in quanto, in particolare:

- consente di trasferire gli obiettivi di rischio definiti a livello complessivo di Banca/Gruppo alle funzioni cosiddette risk taker coinvolte nella fase di assunzione dei rischi;

- $\quad$ permette, attraverso l'attivazione di opportune soluzioni di monitoraggio, di prevenire superamenti di soglie critiche che potrebbero compromettere il rispetto dei valori di risk appetite definiti a livello complessivo di Banca/Gruppo.

\subsection{Strumentazione, dati e data governance}

In termini di strumentazione a supporto del processo RAF, le prassi di mercato evidenziano che il pacchetto Office risulta ad oggi lo strumento di riferimento per la calibrazione delle metriche RAF.

Ciò nonostante, il maggior livello di esposizione verso alcune tipologie di rischio e il grado di complessità informativa gestita ha portato gli istituti di grandi dimensioni a dotarsi di strumenti più sofisticati come sistemi analitici di rischio e tool di

${ }^{7}$ Circolare 285 di Banca d'Italia, Parte I, Titolo IV, Capitolo 3, Sezione I 
simulazione dedicati. A questo proposito in particolare, gli investimenti effettuati nell'ultimo biennio sono stati principalmente indirizzati ad adottare strumentazione in grado di gestire una vasta mole di simulazioni in scenari base e di stress, anche nella logica di valorizzare sinergie con altri processi (in particolare il Recovery Plan per il quale è espressamente richiesto di effettuare analisi di scenario sistemico, idiosincratico e combinato).

L'azione della Vigilanza, che ha interessato approfondimenti mirati sul tema in alcune realtà anche italiane, si è ispirata in proposito ai principi emanati nell'ambito del Comitato di Basilea e contenuti in un paper dedicato (Principles for effective risk data aggregation and risk reporting), indirizzato inizialmente alle Banche di maggiore rilevanza sistemica, e in seguito utilizzato come indicazione cui tendere anche per le altre Banche fatti salvi i principi di proporzionalità.

\subsection{Approccio alla comunicazione, monitoraggio e reporting}

Dal momento in cui la banca ha definito il proprio risk appetite, è necessario delineare le più appropriate modalità di comunicazione della propensione al rischio nei confronti, da un lato, della compagine aziendale e, dall'altro, degli stakeholder esterni.

Al momento, il sistema bancario italiano non evidenzia una modalità univoca di comunicazione del risk appetite, ed anche il livello di dettaglio dei cosiddetti Risk Appetite Statement ${ }^{8}$ varia da banca a banca, con livelli differenziati di analiticità e disclosure.

Inoltre il risk appetite deve essere sottoposto a revisione periodica. Questo aspetto è particolarmente importante quando il modello di business della banca è sottoposto a sollecitazioni esterne e, eventualmente evolve in risposta al contesto di riferimento. È necessario pertanto definire una serie di attività di monitoraggio, di competenza di norma del risk management, per accertare il grado di coerenza dell'operatività con il risk appetite.

L'attività di monitoraggio è funzionale alla verifica della coerenza del risk profile della Banca con il RAF e alla relativa rendicontazione agli Organi aziendali preposti e alle strutture operative interessate dal processo.

Le principali prassi di mercato sono di norma riconducibili all'adozione e allo sviluppo di appositi cruscotti di monitoraggio.

Nonostante l'attuale livello di sofisticazione delle infrastrutture di misurazione e monitoraggio dei rischi, può talvolta risultare complesso rappresentare in modo chiaro e completo la posizione di rischio aggregata oppure il dettaglio di alcune delle tipologie di rischi assunti (ad esempio, i rischi insiti in alcuni segmenti di business non-core, seppur profittevoli per un certo periodo).

Al fine di tenere conto dell'evoluzione dell'operatività, delle strutture organizzative, delle metodologie e degli strumenti di analisi disponibili, le banche oggi sottopongono a continuo aggiornamento il proprio sistema di reporting. Tale aggiornamento tiene inoltre conto di quanto previsto dai principi di Basilea in materia di risk data aggregation \& risk reporting, i quali invitano le banche a predisporre documentazione di reporting che:

- rifletta l'esposizione al rischio in modo accurato e puntuale e trasmetta le informazioni sul profilo di rischio in modo chiaro, sintetico e coerente con le esigenze informative dei destinatari;

- copra tutti i rischi materiali, permettendo l'identificazione di vulnerabilità emergenti;

- $\quad$ sia efficacemente supportata dalle funzionalità di trattamento dati.

\subsection{Meccanismi di escalation}

Come specificato dalle Disposizioni di Vigilanza, nel RAF le banche sono chiamate a definire le procedure e gli interventi gestionali da attivare nel caso in cui sia necessario ricondurre il livello di rischio entro l'obiettivo o i limiti prestabiliti, normando in particolare gli interventi gestionali da adottare al raggiungimento della soglia di tolleranza (ove definita).

I meccanismi di escalation in genere sono differenziati in base alla tipologia dell'indicatore RAF (in termini di distinzione, qualora prevista, tra indicatori di I livello e altri indicatori), alla gravità di superamento dei valori di riferimento (appetite, tolerance, capacity e se previsti valori early warning) e della natura di rischio coinvolto (rischi quantificabili e non).

In alcune realtà la struttura dei limiti del RAF può anche dipendere dalla governance della Banca (es. sistemi duali), con conseguenti differenze nella strutturazione dei limiti del RAF, di reportistica e di azioni conseguenti in caso di sforamento delle soglie definite.

\section{Considerazioni finali, best practices e scenari evolutivi}

La commissione RAF di AIFIRM, attraverso meeting, confronti ed analisi dei risultati della survey sottoposta alle varie banche, ha avuto l'opportunità di analizzare la situazione as is del sistema bancario italiano in tema di implementazione del RAF e di individuare le best practices e le principali prospettive evolutive ad oggi ipotizzabili per gli anni a venire.

Per le banche attuare e consolidare un Risk Appetite Framework efficace costituisce un'opportunità da cogliere per rafforzare la consapevolezza sui rischi e promuovere un'adeguata cultura del rischio, come anche richiesto dal Supervisor, al fine di

\footnotetext{
${ }^{8}$ Il Comitato di Basilea nel documento "Corporate governance principles for banks" definisce il Risk Appetite Statement (RAS) come segue: "The written articulation of the aggregate level and types of risk that a bank will accept, or avoid, in order to achieve its business objectives. It includes quantitative measures expressed relative to earnings, capital, risk measures, liquidity and other relevant measures as appropriate. It should also include qualitative statements to address reputation and conduct risks as well as money laundering and unethical practices."
} 
indirizzare la gestione verso il conseguimento di una profittabilità sostenibile e una solida crescita del business nel lungo termine.

Nel Position Paper sono state indicate le principali componenti attese di un Risk Appetite Framework, tenendo conto delle prassi di mercato oltre che delle richieste regolamentari.

Dalle analisi svolte, e in particolare dai risultati del questionario sottoposto ai partecipanti alla Commissione, è emerso un quadro variegato in termini di grado di maturità e di differenze nell'approccio utilizzato.

In particolare, in taluni ambiti sono state riscontrate delle significative convergenze, per esempio riguardo alle tipologie o classi di rischio considerate, come in tema di indicatori utilizzati per l'adeguatezza patrimoniale.

In altri ambiti, invece, sono state evidenziate differenze d'impostazione, ad esempio in relazione ai ruoli e responsabilità delle varie funzioni rispetto ai vari obiettivi del processo RAF, o in relazione al coordinamento con altri processi di governance della Banca.

Nei casi di disallineamento, dove possibile, è stato anche tracciato un indirizzo ispirato alle best practices e allineato alle richieste normative, ma in taluni casi va sottolineato e anche valorizzato il fatto che il Risk Appetite Framework rimane un esercizio specifico di ogni Banca che in una cornice comune di obiettivi, può essere sviluppato in modo differente per rispecchiare le specificità dei diversi business model, tenuto conto anche del citato principio di proporzionalità.

In ogni paragrafo del Position Paper, dopo la descrizione introduttiva delle tematiche oggetto di analisi, riportate anche sinteticamente in questo articolo, vengono presentate le best practices osservate al fine di fornire uno spunto di riflessione e un'indicazione pratica per l'implementazione del RAF.

In particolare vengono rappresentate le best practice osservate in termini di:

1. articolazione del processo nelle diverse fasi individuate, con focus sulle tempistiche e sulle attività svolte dai diversi attori coinvolti;

2. principali responsabilità di attribuite dalle Banche, come prescritto dalla normativa di riferimento, all'organo con funzione di supervisione strategica, all'organo con funzione di gestione, all'organo con funzione di controllo, alla Funzione di controllo dei rischi, ed alla Funzione di revisione interna;

3. suddivisione delle attività operative tra le funzioni di controllo dei rischi, $\mathrm{P} \& \mathrm{C}$ e CFO, business, anche in relazione alla calibrazione delle metriche RAF;

4. coerenza tra il processo RAF, il Budget, l'ICAAP, il Recovery Plan ed il processo di governo delle operazioni di maggior rilievo: in tutti i casi sono presenti dei requisiti normativi relativi al coordinamento dei processi, che sono approfonditi al fine di individuare i punti di contatto dei diversi processi;

5. principali indicatori utilizzati dalle Banche anche in riferimento ai minimum indicator segnalati dall'EBA nell'ambito della normativa di Recovery Plan;

6. approcci adottati per la calibrazione delle metriche RAF con riferimento alle diverse classi di rischio e tenuto conto delle diverse definizioni di capacity, appetite, tolerance che ricomprendono solitamente il riferimento a indicazioni normative, analisi di sensitivity, scenario, ipotesi judgmental, analisi di trend storici;

7. diverse modalità con cui le banche definiscono il sistema dei risk limit in termini di dettaglio di declinazione, discriminando tra banche di piccole dimensione e realtà più complesse, e approccio alla calibrazione, identificando $i$ criteri adottati per individuare il sistema di cascading;

8. input informativi per l'alimentazione del processo di calibrazione delle metriche RAF;

9. comunicazione, monitoraggio, e reporting, discriminando tra informativa interna ed esterna alla banca, rappresentando la possibile articolazione dei cruscotti di monitoraggio, e analizzando i diversi approcci utilizzati per il reporting agli Organi aziendali, alle Direzioni o ai livelli organizzativi immediatamente successivi o alle funzioni risk taker;

10. approcci utilizzati sia dalle realtà di maggiore dimensione che dalle banche più piccole in termini scelte rispetto ai meccanismi di escalation e attivazione di piani di rientro.

La Commissione RAF dell'AIFIRM, in considerazione del fatto che è lecito attendersi una costante evoluzione del RAF che tenga conto delle modifiche interne alla banca e del contesto competitivo esterno, ha inoltre identificato alcuni ambiti di potenziale ulteriore sviluppo che potrebbero essere affrontati dalle banche italiane nei prossimi anni, descritti nel dettaglio nel Position Paper, in particolare:

- ulteriore integrazione del RAF nei processi strategici, con finalità di consolidare il RAF come strumento di supporto alle decisioni strategiche, anche in relazione a temi di attualità quali operazioni di aumento di capitale, smaltimento di non performing loans, impatto dei tassi bassi o negativi;

- $\quad$ approfondimento e sviluppo di metodologie di analisi su tipologie di rischio emergenti, ampliando lo spettro di rischi coperti dal RAF, in coerenza con l'evoluzione del business bancario e del contesto competitivo. In particolare i principali nuovi rischi identificati riguardano il Cyber \& Fraud Risk, il Compliance Risk, lo Strategic Risk e il Model Risk; 
- rafforzamento della coerenza tra RAF e risk policy, con particolare riferimento al cascading del RAF sulle principali business combinations (BU e Legal entity), e alla determinazione del framework di limiti con maggiore integrazione rispetto ai processi gestionali e operativi di business;

- $\quad$ rafforzamento della strumentazione e consolidamento dei processi di data aggregation e reporting.

\section{Diego Onorato}

\section{Bibliografia}

- AIFIRM (2017), Il ruolo del RAF nella governance delle banche

I coordinatori della Commissione Aifirm:

Marina Brogi, Diego Onorato, Antonio Ricciardi

I contributori ai lavori:

Stefano Giampieri, Jason Kessler, Fernando Orsetti, Alessio Pentola, Mario Vellella,

e tutti i partecipanti alla Commissione

Pubblicazioni citate nel lavoro:

- $\quad$ EBA Guidelines on Internal Governance (EBA, 27 settembre 2011);

- Intensity and Effectiveness of SIFI Supervision - Progress report on implementing the recommendations on enhanced supervision (Financial Stability Board, 27 ottobre 2011);

- $\quad$ Core Principles for Effective Banking Supervision (Comitato di Basilea, settembre 2012);

- $\quad$ Principles for effective risk data aggregation and risk reporting (Comitato di Basilea, gennaio 2013);

- Direttiva del Parlamento europeo e del Consiglio 2013/36/UE (26 giugno 2013);

- Regolamento del Parlamento europeo e del Consiglio 2013/575/UE (26 giugno 2013);

- Principles for an effective Risk Appetite Framework (Financial Stability Board, 18 novembre 2013);

- Disposizioni di Vigilanza per le Banche (Circolare Banca d'Italia n. 285 del 17 dicembre 2013);

- Guidelines on common procedures and methodologies for the supervisory review and evaluation process (SREP) (EBA, 19 dicembre 2014);

- $\quad$ Corporate governance principles for banks (Comitato di Basilea, luglio 2015);

- $\quad$ SSM supervisory statement on governance and risk appetite (ECB, giugno 2016). 


\title{
Ricostruzione di superfici di volatilità mediante l'utilizzo di reti neurali auto-associative: un caso studio basato sull'analisi non lineare delle componenti principali
}

di Ottavio Caligaris (Università di Genova) - Pier Giuseppe Giribone (Banca Carige) - Marco Neffelli (Università di Genova) ${ }^{1}$

\begin{abstract}
Under the log-normal framework, it is not possible to correctly derive volatility surfaces when interest rates are negative. In fact, volatility surfaces are obtained by reverse engineering on the Black formula, which involves logarithmic terms; since it takes the observed prices of available financial instruments and the interest rates as main inputs, they both cannot be negative. Therefore, negative interest rates are responsible of generating this Data-Missing problem. Data-Missing problems are often circumvented with interpolation; unfortunately, here a more complex approach is needed since a too large portion of the surface is missing.

This work proposes the use of auto-associative neural networks in order to reconstruct the volatility surface. The novelty of the approach is to capture the non-linear relationships of the volatility surface through an analysis of non-linear principal components, which is incorporated in the neural networks. We demonstrate the ability of this methodology of completely reconstructing the volatility surfaces, particularly when interest rates are negative and the classical methodologies fail to describe the exact trend embedded in the Black volatility surface.

The paper is structured in four sections: section 2 illustrates the methodology of non-linear principal component analysis and its application in the auto-associative neural networks framework; section 3 validates the MATLAB code considering a complex analytic surface; section 4 demonstrates the robustness of the implemented approach with an empirical study in which the log-normal volatility surface for European swaption is reconstructed; section 5 concludes.
\end{abstract}

\section{Key Words:}

Auto-associative neural network (AANN), Non Linear Principal Component Analysis (NLPCA), Machine Learning (ML), volatility surface reconstruction, missing-data problem, swaption volatility cube

\section{Introduzione}

La presenza di tassi di interesse negativi nei maggiori mercati finanziari rende problematica la derivazione delle superfici di volatilità log-normali alla Black. Tali superfici vengono ottenute applicando un processo di ingegneria inversa sulla formula di Black, utilizzando come input principali le quotazioni di strumenti finanziari osservati sui mercati e i tassi di interesse. Quest'ultimi, al fine di garantire la corretta derivazione delle superfici di volatilità, devono essere positivi, data la presenza di termini logaritmici nella formula di Black. Qualora i tassi di interesse siano negativi, ci si trova di fronte ad un c.d. MissingData Problem, dato che una porzione consistente della superficie di volatilità risulta mancante. I problemi della ricostruzione di dati mancanti sono spesso risolti da metodologie tradizionali, quali l'interpolazione, che purtroppo in questo caso risultano grossolane e non idonee a riprodurre una vasta porzione di superficie.

Questo lavoro propone la ricostruzione delle superfici di volatilità mediante l'utilizzo di reti neurali auto-associative, strumenti adatti per un'analisi non lineare delle componenti principali dei dati. Si dimostra che tali modelli permettono di ottenere una ricostruzione completa delle superfici sopra citate, soprattutto in presenza di tassi di interesse negativi, laddove la modellistica tradizionale non risulta in grado di derivare la superficie di volatilità di Black.

Lo studio è organizzato in quattro sezioni: la sezione 2 presenta la modellistica dell'analisi non lineare delle componenti principali e del loro impiego tramite reti neurali auto-associative; la sezione 3 consiste nel validare il codice MATLAB su un esempio di superficie analitica piuttosto complessa; la sezione 4 presenta uno studio empirico riguardante la ricostruzione della superficie di volatilità alla Black nel caso di swaption europee; la sezione 5 propone le conclusioni.

\section{L'analisi delle componenti principali impiegando reti neurali autoassociative}

L'analisi delle componenti principali (Principal Component Analysis abbreviato in PCA) fornisce uno strumento per studiare un insieme di dati individuando relazioni lineari tra essi.

Si supponga di dover esaminare un insieme di dati raccolti in una matrice $P$. Le righe della matrice rappresentano le osservazioni e ciascun elemento di ogni riga rappresenta una caratteristica dell'entità in osservazione. Per semplicità supporremo che le caratteristiche in esame siano tre per cui:

${ }^{1}$ Articolo sottoposto a doppio referaggio anonimo, pervenuto il 3/07/2017 e accettato il 17/11/2017 
$P=\left(\begin{array}{lll}x_{1} & y_{1} & z_{1} \\ x_{2} & y_{2} & z_{2} \\ x_{3} & y_{3} & z_{3} \\ \cdots & & \\ x_{n} & y_{n} & z_{n}\end{array}\right)=\left(\begin{array}{c}P_{1} \\ P_{2} \\ P_{3} \\ \cdots \\ P_{n}\end{array}\right)=\left(\begin{array}{lll}x & y & z\end{array}\right)$

Supponiamo i dati costituenti la matrice in forma normalizzata, nel caso non lo fossero sostituiamo ad $x$ il vettore $\frac{x-\mu_{x}}{\sigma_{x}}$ essendo $\mu_{x}$ la media e $\sigma_{x}$ la deviazione standard di $x$.

Tipicamente $\left(x_{k}, y_{k}, z_{k}\right)$ potrebbe rappresentare un punto del grafico di una funzione $z=f(x, y)$ di due variabili.

Mediante la PCA possiamo mettere in evidenza eventuali dipendenze lineari, o approssimabili linearmente, tra $x, y$ e $z$ in modo da caratterizzare il comportamento di $f$.

Ciò può essere fatto cercando due matrici $A$ e $B$ che consentano di proiettare i dati su uno spazio di dimensione inferiore, nel nostro caso 3, mediante la trasformazione lineare $R=A P$ e di ricostruire i dati iniziali mediante una seconda trasformazione lineare individuata dalla matrice $B$ in modo che $B A P$ approssimi al meglio $P$, in modo che la quantità Err $=\|P-B A P\|^{2}$ sia minima.

Le matrici $A$ e $B$ possono essere individuate utilizzando gli autovettori della matrice di correlazione dei dati, ma si prestano anche ad essere stimate utilizzando un'opportuna rete neurale. Infatti, assegnata una terna di dati $P$, possiamo schematizzare il procedimento per ottenere $B A P$ come una rete neurale costituita da neuroni con funzione di attivazione uguali all'identità: $s(x)=x$ (Fig. 1)
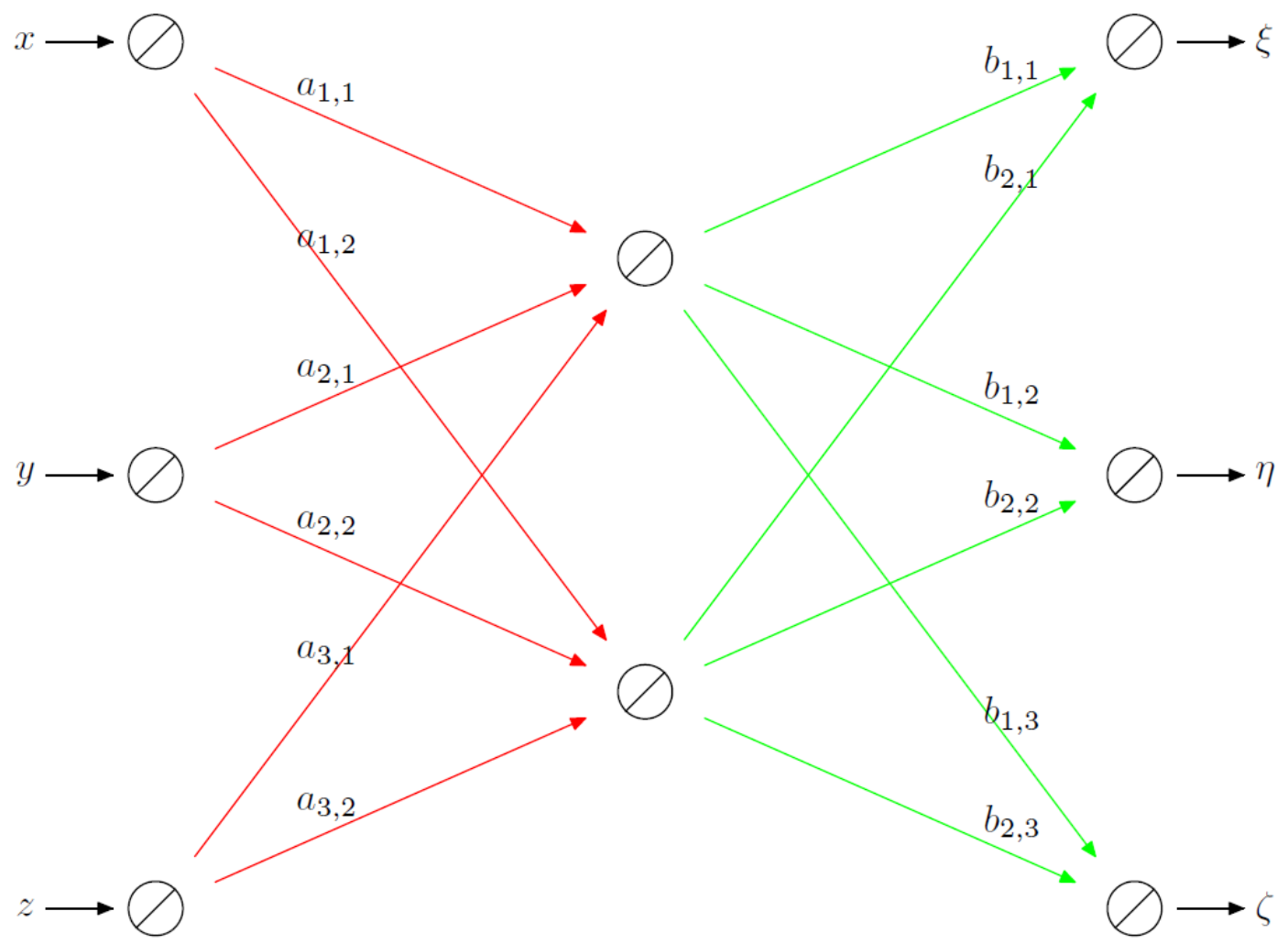

Figura 1. Architettura neurale per la PCA lineare

Il primo strato della rete rappresenta i dati in ingresso $P$, il secondo rappresenta i dati proiettati su uno spazio di dimensione inferiore, mentre il terzo strato rappresenta l'output che dovrà essere confrontato con $P$; la rete poi si può addestrare in modo da minimizzare la differenza tra input ed output. 
I pesi assegnati alle connessioni tra primo e secondo strato sono definiti dalla matrice $A$, mentre $B$ è la matrice dei pesi da assegnare alle connessioni tra secondo e terzo strato. Le matrici $A$ e $B$ che minimizzano la differenza $E r r=\|(x, y, z)-(\xi, \eta, \zeta)\|^{2}$ si possono trovare, come per una rete neurale standard propagando all'indietro l'errore, il che corrisponde all'applicazione del metodo del gradiente per l'ottimizzazione di $\mathrm{Err}$.

L'idea introdotta nell'Analisi delle Componenti Principali Non-lineare, (NLPCA) si riduce essenzialmente nel sostituire alla funzione lineare definita dal prodotto di due matrici una funzione non-lineare.

La non-linearità è indotta dall'applicazione di una funzione di attivazione di forma sigmoidale alla somma degli ingressi di ciascun neurone.

Quel che si ottiene è una Rete Neurale Autoassociativa (AANN) che deve essere addestrata in modo che la differenza tra dato in ingresso e dato in uscita sia minima. Pertanto si cerca di determinare $F$ e $G$, funzioni tali che Err $=\|P-G(F(P))\|^{2}$ risulti minima, essendo $F$ una funzione che trasporta il dato $P$ su uno spazio di dimensione inferiore e $G$ una funzione che ricostruisce il dato, $F$ e $G$ sono funzioni definite per mezzo di una rete neurale autoassociativa: la situazione è descritta nella figura 2 .

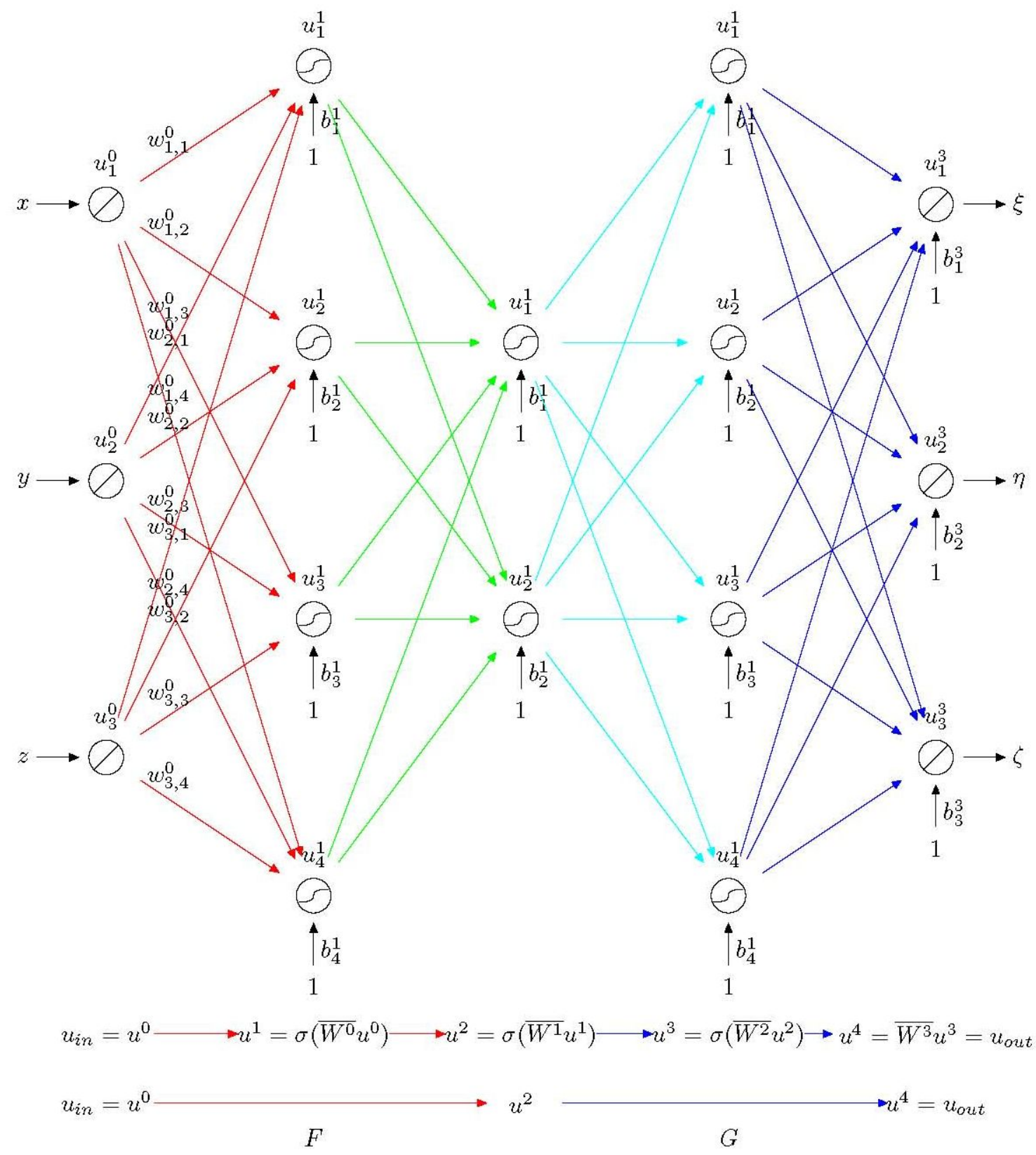




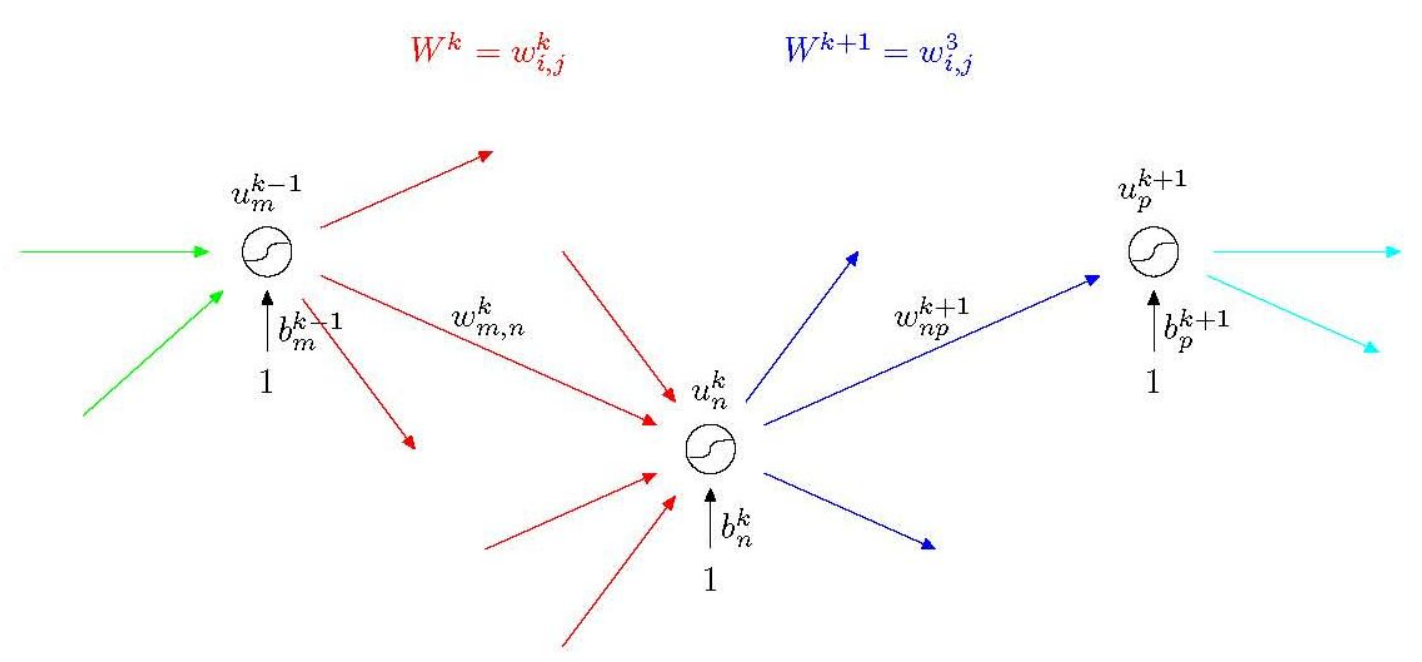

Figura 2. Architettura neurale impiegata per la PCA non lineare

La rete viene addestrata minimizzando Err mediante il classico metodo del gradiente; la struttura di Err è tale che le operazioni da compiere sono localizzate per ogni neurone e possono essere attuate mediante un algoritmo di facile impiego, che prende il nome di back-propagation. Di fatto la rete definisce una funzione che è una ottima approssimazione dell'identità se calcolata sui dati in esame. Inoltre recepisce in qualche senso la struttura dei dati e consente di ricostruire eventuali dati mancanti; inoltre, dal momento che lo strato centrale è costituito soltanto da 2 neuroni, si crea un "collo di bottiglia" che in qualche modo proietta $\mathrm{i}$ dati, originariamente in $\mathfrak{R}^{3}$, su uno spazio bidimensionale. Per comprendere come agisce, proponiamo un semplice esempio. Consideriamo $\mathrm{i}$ punti in $\mathfrak{R}^{3}$ aventi coordinate $(x, y, 2 x+5 y)$ per $x \in[1,8]$ ed $y \in[1,14]$ ed alteriamo i valori della terza coordinata con un valore casuale ragionevolmente piccolo, in modo che la superficie generata conservi l'andamento, anche se perturbato, del piano $z=2 x+5 y$. Inoltre eliminiamo circa il $30 \%$ dei valori di $z$. Otteniamo una superficie nella quale si riconosce l'andamento lineare, che tuttavia è fortemente perturbata e manca di numerosi dati (Figura 3). Addestrando con i valori ottenuti una rete neurale auto-associativa del tipo prima descritto, otteniamo in uscita la superficie mostrata in figura 4, nella quale è messo in evidenza l'andamento lineare. In aggiunta viene fornito un valore calcolato anche nei casi in cui mancavano i dati. Nella figura 5 è riportata la ricostruzione corretta della superficie di partenza con tecnica NLPCA.

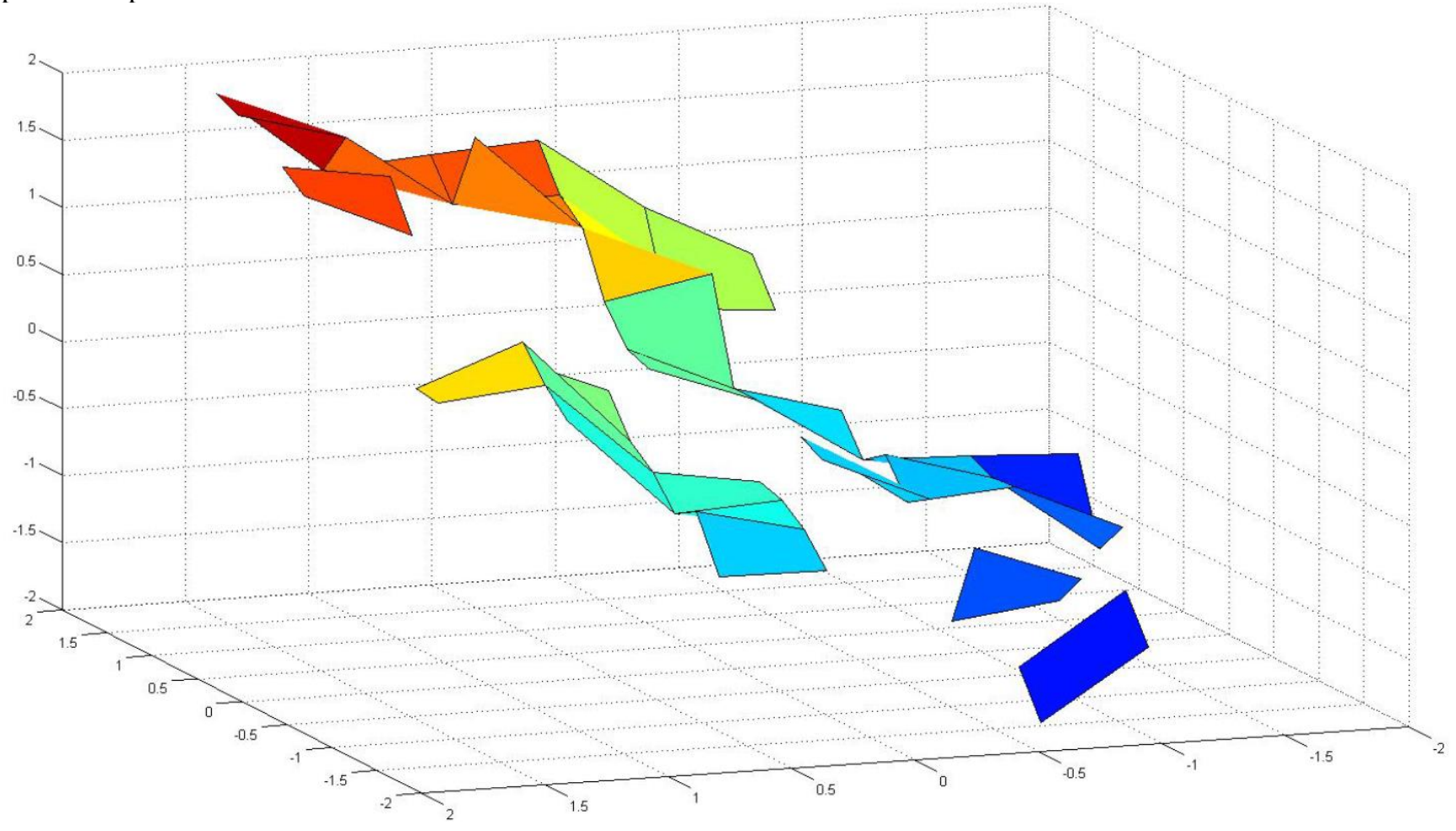

Figura 3. La superficie risultante dopo aver eliminato il $30 \%$ dei valori dal piano iniziale e aver introdotto nei rimanenti una componente aletoria 


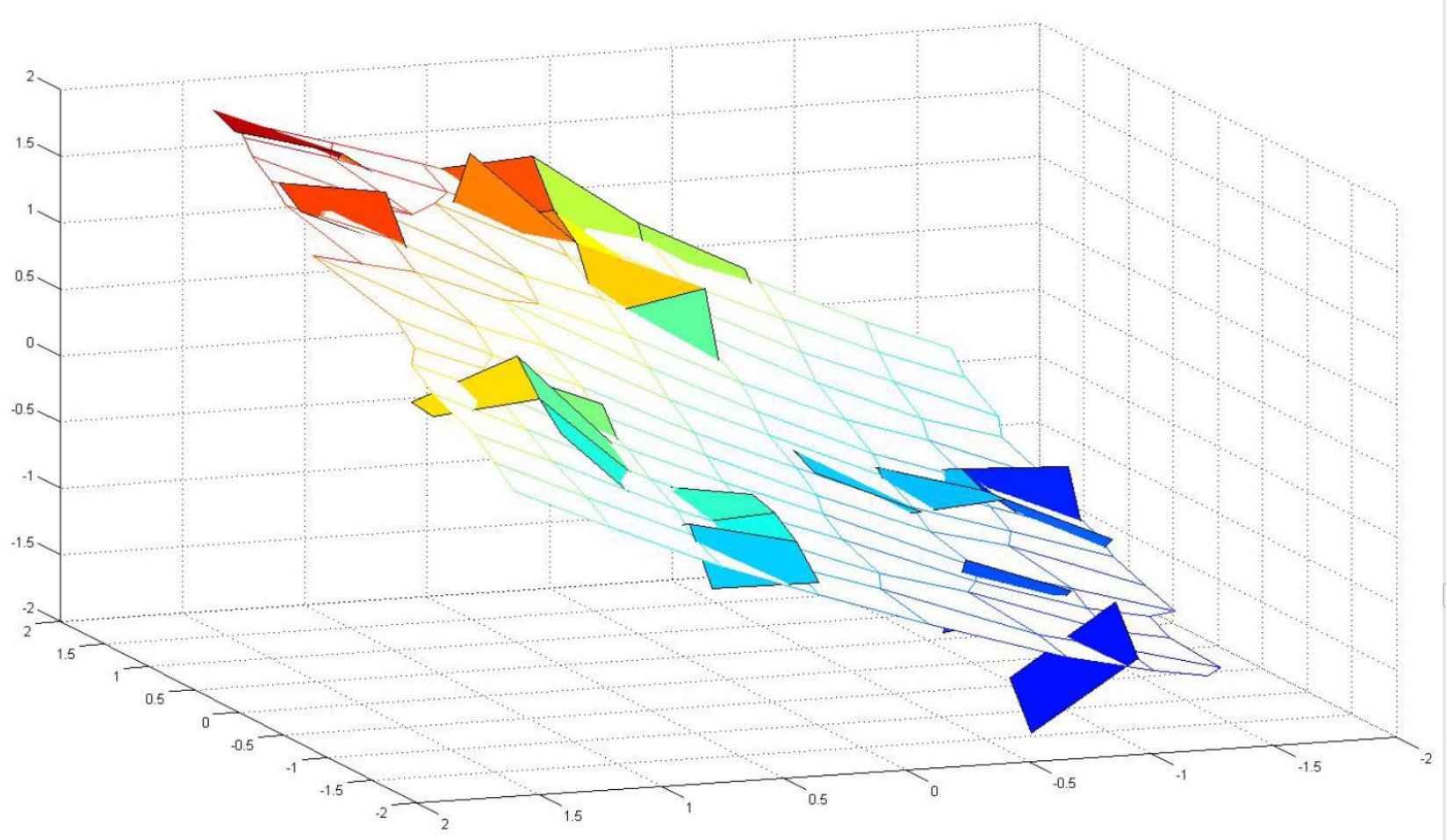

Figura 4. Un'immagine della ricostruzione della AANN dopo 1.000 epoche

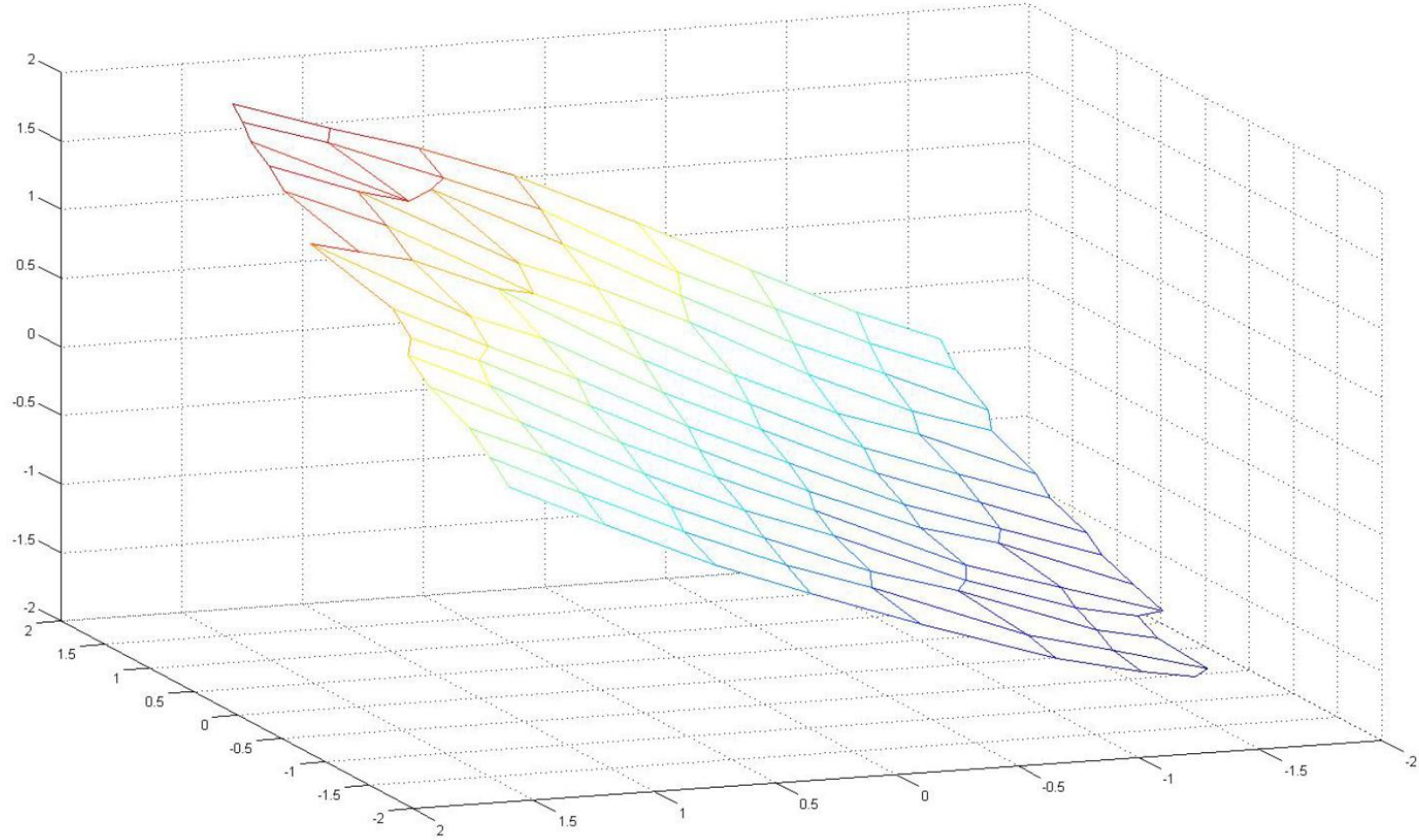

Figura 5. La ricostruzione del piano originale con reti neurali auto-associative 


\section{Validazione del codice Matlab}

Al fine di validare il codice effettuiamo una prova di ricostruzione di una superficie abbastanza complessa, definita da:

$f(x, y)=\frac{\sin \left(\sqrt{x^{2}+y^{2}}\right)}{\sqrt{x^{2}+y^{2}}} \operatorname{con} f(0,0)=1$

Come si vede nelle figure 6 - 7, nonostante la significativa numerosità di dati mancanti, l'algoritmo di Machine Learning codificato dagli autori è stato in grado di ricostruire correttamente la funzione. Nel prossimo paragrafo verrà applicata la metodologia ad un caso inerente il mercato finanziario.
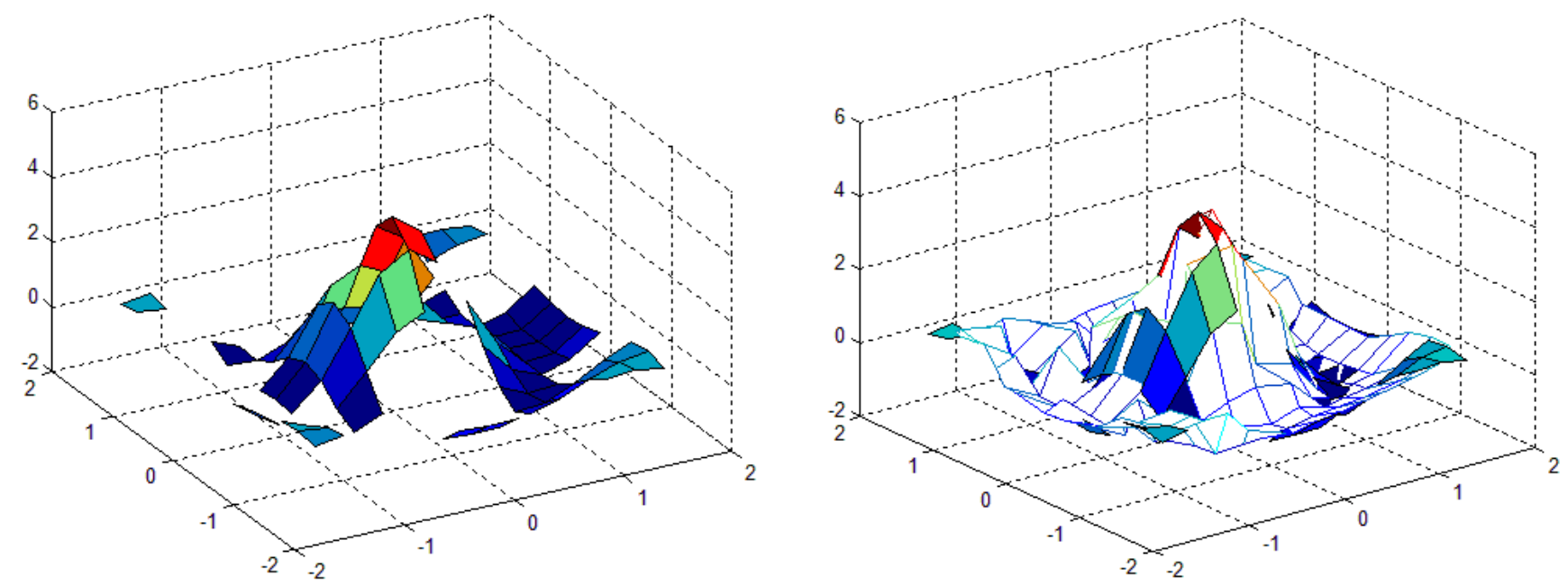

Figura 6 - 7. La ricostruzione della superficie di prova con le AANN

\section{Ricostruzione della superficie delle volatilità di Black per Swaption}

Si consideri la superficie di volatilità at-the-money (ATM) delle swaption osservata sul mercato il 29/07/2016, periodo nel quale i tassi d'interesse erano fortemente negativi nell'area Euro.

Nella Tabella 1 sono riportati i valori della volatilità log-normale nelle dimensioni tenor $(\tau)-$ maturity $(T)$ e per strike ATM. Come si evince sia dalla matrice (Tabella 1), sia dalla sua rappresentazione grafica (Figura 8), grandi porzioni di tale superficie non sono quotate (N.D.).

Il motivo di tale mancanza di dati è riconducibile al fatto che, con l'eccezione del punto in corrispondenza di $\tau=T=10$ anni, si riscontra l'impossibilità da parte del framework di pricing lognormale di trattare tassi d'interesse negativi.

Qualora sia necessario esprimere un valore numerico laddove manchi, è necessario ricorrere ad una metodologia complessa di ricostruzione dati, tipo quelle di Machine Learning (ML).

All'interno di questo contesto, si propone l'approccio basato sull'analisi non lineare delle componenti principali (NLPCA), descritto nei paragrafi precedenti, al fine di ricostruire la superficie di volatilità.

\begin{tabular}{|c|c|c|c|c|c|c|c|c|c|c|c|c|c|c|}
\hline Term & 1 YR & 2 YR & 3 YR & 4 YR & 5 YR & 6 YR & 7YR & 8 YR & 9 YR & 10 YR & 15 YR & 20 YR & 25 YR & 30 YR \\
\hline 1 YR & N.D. & N.D. & N.D. & N.D. & N.D. & N.D. & N.D. & 210,54 & 202,2 & 159 & 98,99 & 92,16 & 92,65 & 95,93 \\
\hline 2 YR & N.D. & N.D. & N.D. & N.D. & N.D. & N.D. & 205,26 & 199,82 & 151 & 131 & 92,96 & 88,24 & 90,58 & 94,63 \\
\hline 3 YR & N.D. & N.D. & N.D. & N.D. & N.D. & 203,67 & 187,04 & 139,36 & 120,7 & 110,4 & 86,62 & 84,83 & 88,58 & 93,1 \\
\hline 4 YR & N.D. & N.D. & N.D. & N.D. & 200,33 & 151,43 & 122,25 & 108,01 & 99,8 & 94,73 & 81,41 & 81,7 & 85,05 & 90 \\
\hline 5 YR & N.D. & N.D. & 207,57 & 177,2 & 127,19 & 107,07 & 97,07 & 90,73 & 86,74 & 84,64 & 77,24 & 79,01 & 83,09 & 88,37 \\
\hline 7 YR & N.D. & 112,32 & 95,62 & 86,17 & 81,25 & 77,76 & 75,51 & 74,22 & 73,79 & 73,94 & 72,39 & 76,06 & 80,53 & 86,29 \\
\hline 10 YR & 77,25 & 72,05 & 70,94 & 69,74 & 69,65 & 70,07 & 71,42 & 72,7 & 74,26 & N.D. & 78,52 & 84,33 & 90,87 & 98,74 \\
\hline 15 YR & 85,69 & 90,9 & 97,75 & 109,17 & 131,88 & 221,68 & 218,45 & 136,97 & 168,3 & 70,35 & 97,64 & 94,2 & 99,61 & 114,8 \\
\hline
\end{tabular}

Tabella 1. Valori in percentuale della matrice di volatilità ATM di Black per Swaption. Fonte: Bloomberg® 


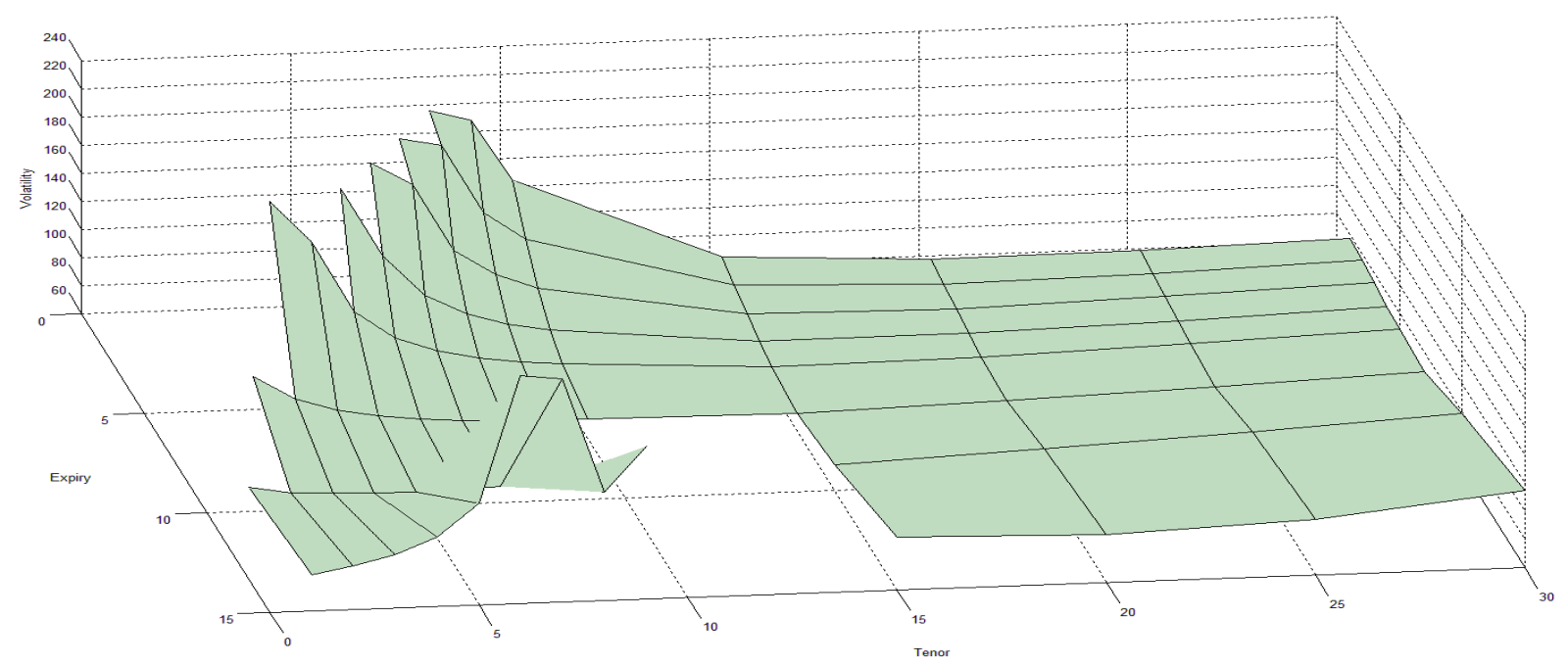

Figura 8. Rappresentazione grafica della superficie di volatilità ATM di Black per Swaption

Le Figure 9 e 10 rappresentano i punti ricostruiti dalla rete auto-associativa della superficie di volatilità log-normale di Black.

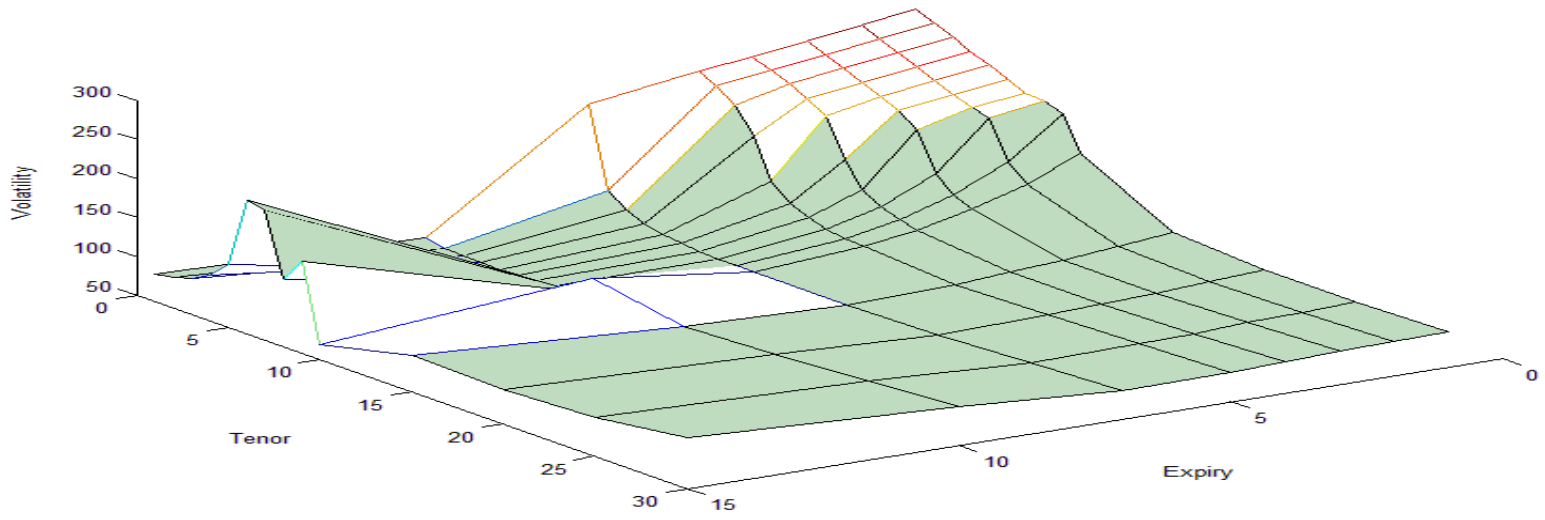

Figura 9. La ricostruzione della superficie di volatilità mediante reti neurali auto-associative

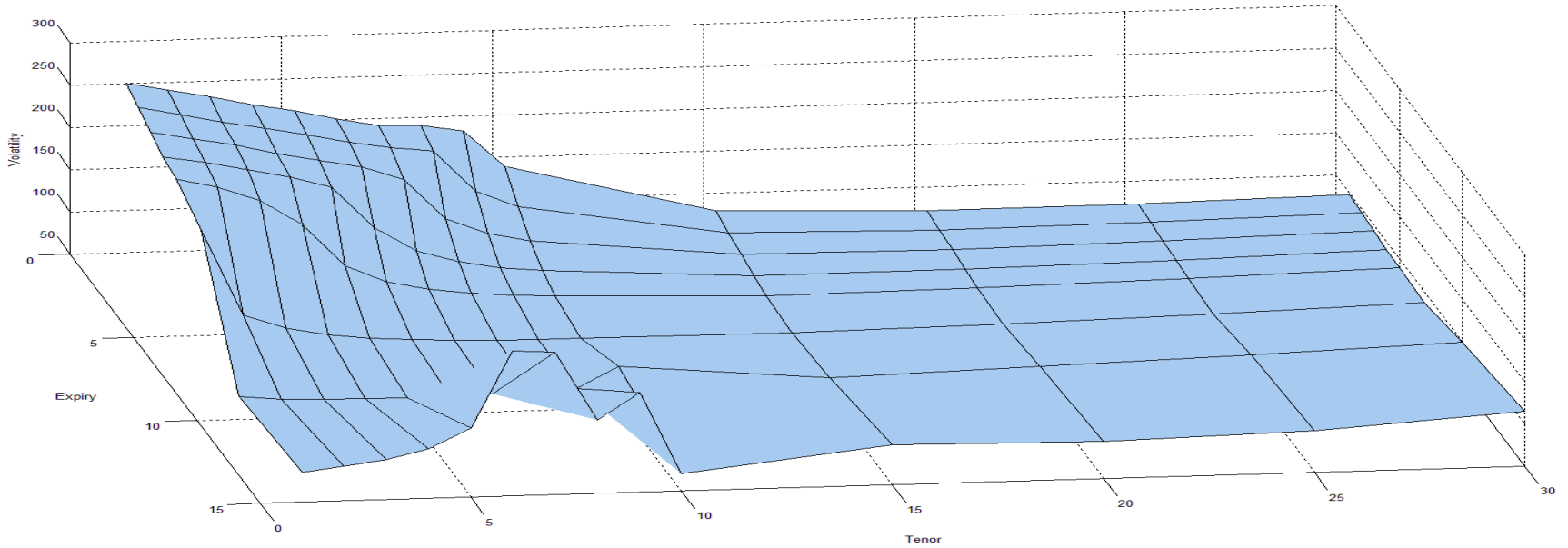

Figura 10. La superficie di volatilità ricostruita da impiegare in un framework log-normale di pricing 


\section{Conclusioni}

L'articolo propone l'impiego di reti neurali autoassociative basate sull'analisi non-lineare delle componenti principali al fine di risolvere il problema di dati mancanti, che possono essere riscontrate nelle superfici di volatilità log-normali in presenza di tassi di interesse negativi. Dopo aver introdotto il modello e averne testato il funzionamento su superfici analitiche predefinite, si mette in risalto il proficuo utilizzo della metodologia con dati reali di mercato. Nello specifico, si prende ad esempio il caso della superficie di volatilità alla Black per le swaption dell'area euro al 29/07/2016, condizionata da un'ampia moltitudine di dati mancanti a causa dei tassi di interesse fortemente negativi. L'applicazione del framework di Machine Learning permette di ricostruire adeguatamente la superficie di volatilità log-normale, fornendo all'analista le informazioni necessarie per condurre correttamente le funzioni di risk management, option pricing e portfolio allocation.

La NLPCA è una metodologia estremamente versatile che, identificando e rimuovendo correlazioni tra i dati, consente di ridurre la dimensionalità di un problema permettendo un'analisi dei dati più efficiente.

Sotto questo aspetto, si reputa utile impiegare la selezione delle componenti principali di un sistema di mercato complesso a fini di trading.

\section{Ottavio Caligaris, Pier Giuseppe Giribone e Marco Neffelli}

\section{Bibliografia}

[1] H. C. Chen, Visualization of financial time series by linear principal component analysis and nonlinear principal component analysis, MSc Thesis in Financial Mathematics and Computation, University of Leicester (2014)

[2] M. Scholz, Validation of nonlinear PCA, Neural Processing Letters, 36,1, 21-30 (2012)

[3] M. Scholz, M. Fraunholz, J. Selbig, Nonlinear principal component analysis: neural network models and applications, Book Chapter in "Principal manifolds for data visualization and dimension reduction", 58, 44-67, Springer Berlin (2007)

[4] M. Scholz, Analysing periodic phenomena by circular PCA, Proceedings of the Conference on Bioninformatics Research and Development BIRD’07, 4414, 38-47, Springer-Verlag (2007)

[5] M. Scholz, F. Kaplan, C. L. Guy, J. Kopka, J. Selbig, Non-linear PCA: a missing data approach, Bioinformatics, 21, 3887$3895(2005)$

[6] M. Scholz, Nonlinear PCA based on neural networks, Dep. Of Computer Science, Humboldt University Berlin Diploma Thesis (2002)

[7] M. Scholz, R. Vigario, Nonlinear PCA: a new hierarchical approach. Proceedings ESANN (2002)

[8] J. Rachlin, Principal Component Analysis and Extreme Value Theory in Financial Application, Bachelor of Arts Degree, Department of Physics (2006)

[9] M. A. Kramer, Nonlinear Principal Component Analysis using autoassociative neural networks, AIChE Journal, 37, 2, 233-243 (1991)

[10] N. Lawrence, Probabilistic Non-linear Principal Component Analysis with Gaussian Process Latent variables models, Journal of Machine Learning Research, 6 (2005)

[11] O. Caligaris, Le reti neurali, Lettera matematica PRISTEM, 61: 20-28 (2007)

[12] P. G. Giribone, S. Ligato, O. Caligaris, Applicazioni delle reti neurali feed-forward per la ricostruzione di superfici di volatilità, AIFIRM Magazine (Financial Industry Risk Managers Association), 10, 1, 4-19 (2015)

[13] P. G. Giribone, O. Caligaris, Modellizare la curva dei rendimenti mediante metodologie di apprendimento artificiale: analisi e confronto prestazionale tra le tecniche regressive tradizionali e le reti neurali, AIFIRM Magazine (Financial Industry Risk Managers Association), 10, 3, 16-32 (2015)

[14] J. C. Hull, Options, Futures and other derivatives, Pearson-Prentice Hall, Englewood Cliffs, $8^{\text {th }}$ Edition (2012) 


\section{L'applicazione dei nuovi scenari di variazione dei tassi di interesse proposti dal Comitato di Basilea: quali implicazioni per le banche italiane?}

di Igor Gianfrancesco ${ }^{12}$

\section{Abstract}

Risk arising from fluctuations of interest rates can pose a significant threat to a bank's ability to generate earnings and to the adequacy of its capital endowment. An effective risk management process, able to maintain interest rate risk in the banking book (IRRBB) within prudential levels is essential for the overall banking stability, due to the systemic nature of this risk. This paper aims to provide preliminary evidences on the impact of the new six interest rate shock scenarios recently added by the Basel Committee to the current IRRBB regulatory framework, in order to capture the risk arising from changes in level and shape of the yield curve. Furthermore, the study sheds more light on the application of the non-negativity constraint in a period of negative interest rates, based in the recent European Banking Authority's indications. Referred to a sample of 140 Italian commercial banks over the period 2006-2016, the paper shows that the application of the new interest rate scenarios allows to avoid the risk neutrality phenomenon that may occur when the old shock scenarios are used. Overall, Italian commercial banks are exposed to the scenarios associated with the short rates down and the steepener shocks. However, during the recent past years, theirexposure to the former decreased significantly, due to the low interest rate environment level and the consequent application of the non-negativity constraint.

\section{Introduzione}

Il presente contributo si propone di analizzare su un campione di 136 banche commerciali italiane nel periodo 20062016 le implicazioni derivanti dall'introduzione nell'ambito della misurazione dell'esposizione al rischio di tasso di interesse del portafoglio bancario dei sei nuovi scenari di variazione dei tassi di interesse introdotti dal Comitato di Basilea nel documento tecnico dell'aprile 2016, effettuando un confronto con il quadro normativo di vigilanza prudenziale attualmente in vigore. Ciò al fine fornire indicazioni funzionali alla corretta comprensione delle determinanti dell'esposizione al rischio e, più in generale, ad una riflessione sul ruolo dell'Asset \& Liability Management (ALM) in un contesto di riferimento dove le suddette innovazioni regolamentari si inseriscono in un mutato scenario economico e finanziario, che ha condotto ad una modifica delle stesse strategie di $A L M$ delle banche e, quindi, della relazione di interdipendenza tra l'attivo e il passivo di bilancio.

Nell'ultimo periodo, infatti, dal lato dell'attivo si è registrato un incremento dei titoli di stato iscritti nel portafoglio bancario; dal lato del passivo, invece, una differente composizione in termini di forme tecniche con un maggior peso della raccolta a breve termine verso controparti al dettaglio e della raccolta verso la Banca Centrale Europea e un minore ricorso all'emissione di prestiti obbligazionari ${ }^{3}$. A ciò si aggiunge il basso livello dei tassi di interesse, che, oltre a influenzare le stesse strategie di $A L M$ delle banche, ha impattato sulle modalità di calcolo dell'indicatore di rischio regolamentare determinando, a seguito dell'applicazione del vincolo di non negatività, degli effetti distorsivi che hanno dato luogo al fenomeno della neutralità al rischio. Si tratta, in altri termini, di banche non esposte al rischio di tasso di interesse del portafoglio bancario a seguito dell'applicazione delle metodologie di calcolo proposte dal quadro normativo di vigilanza prudenziale attualmente vigente ${ }^{4}$.

I nuovi scenari di variazione dei tassi di interessi definiti dal Comitato di Basilea tengono conto di differenti movimenti del livello e della forma della curva dei rendimenti in aggiunta al già utilizzato spostamento parallelo e sono rappresentati, nello specifico, dal parallel shock up e dal parallel shock down, dallo short rates shok up e dallo short rates shock down, dallo

\footnotetext{
${ }^{1}$ Articolo sottoposto a doppio referaggio anonimo, pervenuto il 5/1/2018 e accettato il 18/02/2018.

${ }^{2}$ Responsabile Ufficio Risk Management Extrabanca e titolare di un contratto di insegnamento integrativo presso la Cattedra di Economia e Gestione degli Intermediari Finanziari (Corso Progredito) dell'Università LUISS Guido Carli di Roma. Le opinioni espresse sono proprie dell'Autore e non vincolano in alcun modo le Istituzioni di appartenenza. Articolo redatto sulla base delle informazioni disponibili al $31 / 12 / 2017$

${ }^{3}$ Tali dinamiche sono desumibili dalle evidenze riportate, con periodicità trimestrale, nel Bollettino Economico della Banca d'Italia alla Tavola contenente i dati relativi alle principali voci di bilancio delle banche italiane. Con riferimento al periodo che va dal maggio del 2011 [Banca d'Italia (2012)] ad agosto 2017 [(Banca d'Italia (2018)] la quota dei titoli detenuti in portafoglio dalle banche ed emessi da amministrazioni pubbliche sul totale attivo è passata dal 5,46\% al 9,68\%; la quota dei depositi verso residenti sul totale del passivo dal $30,13 \%$ al 37,66\%, quella della raccolta verso la BCE dallo $0,91 \%$ al 6,56\% e, infine, quella delle obbligazioni emesse dal $16,62 \%$ al $7,82 \%$. Evidenze sostanzialmente in linea sono riscontrabili anche sul campione di banche utilizzato presente analisi nel periodo 2006-2016 di cui alla Tabella 3 del paragrafo 5 a cui si rimanda per un approfondimento.

${ }^{4}$ Il concetto di neutralità al rischio è stato più volte segnalato e analizzato dalla letteratura di riferimento. Al riguardo si rimanda a Cocozza et al. (2015), Gianfrancesco (2016) e Cerrone et al. (2017).
} 
steepener shock (short rates down e long rates up) e dal flattener shock (short rates up e long rates down). La proposta del Comitato di Basilea è stata considerata nell'ambito del pacchetto globale di riforme presentato dalla Commissione Europea il 26 novembre 2016. Il nuovo paragrafo 5 bis dell'art.98 della Direttiva 2013/36/EU ha conferito, infatti, all'Autorità Bancaria Europea l'incarico di elaborare norme tecniche di regolamentazione per specificare in corrispondenza delle varie valute i sei scenari prudenziali di shock da applicare ai tassi di interesse. Ciò è, successivamente, avvenuto mediante l'emanazione, da parte della stessa Autorità Bancaria Europea, del documento di consultazione dello scorso 31 ottobre 2017 riguardante l'aggiornamento delle linee guida per la misurazione e gestione del rischio di tasso di interesse del portafoglio bancario in cui sono stati recepiti gli scenari, inizialmente, proposti dal Comitato di Basilea. Infine, il $20^{\circ}$ aggiornamento della Circolare 285/2013 della Banca d'Italia del recente 21 novembre 2017 chiarisce che le banche devono considerate anche spostamenti non paralleli della curva dei rendimenti per determinare la propria esposizione al rischio di tasso di interesse del portafoglio bancario richiedendo, per il momento solo implicitamente, l'adozione di tali scenari da parte delle banche italiane nella misurazione della propria esposizione al rischio di tasso di interesse del portafoglio bancario.

Il presente contributo si inserisce in un'area di ricerca che analizza, da differenti prospettive, la robustezza della metodologia di calcolo definita dal Comitato di Basilea nel 2004, e, successivamente, recepita dalla Banca d'Italia prima nella Circolare 263 del dicembre 2010 e poi nella Circolare 285 nel dicembre 2013, per stimare l'esposizione al rischio di tasso di interesse del portafoglio bancario [cfr. Fiori e Iannotti (2007), Entrop et al. (2008), Entrop et al. (2009), Abdymomunov e Gerlach (2014), Cocozza et al. (2015), Cerrone et al. (2017)]. L'AIFIRM ha posto particolare attenzione a tale tematica ospitato vari contributi nella propria Newsletter [cfr. Curcio e Gianfrancesco (2012), Gianfrancesco e Giliberto (2014) e Gianfrancesco (2016)] oltre a predisporre uno specifico Position Paper nell'ambito della consultazione promossa nel 2015 dal Comitato di Basilea, che ha condotto alla stesura del documento tecnico pubblicato ad aprile 2016. In aggiunta alla precedente letteratura il presente contributo fornisce una prima stima dell'impatto sull'esposizione al rischio dei sei nuovi scenari definiti dal Comitato di Basilea. Inoltre, affronta la questione relativa all'applicazione del vincolo di non negatività in un contesto di livello negativo dei tassi di interesse facendo riferimento alle recenti indicazioni fornite dall'Autorità Bancaria Europea nel luglio del 2017.

Le principali evidenze empiriche ottenute mostrano che l'applicazione dei nuovi scenari di variazione dei tassi di interesse proposti dal Comitato di Basilea consente, in generale, di evitare il verificarsi del fenomeno della neutralità al rischio rispetto a quanto si osserva, come già evidenziato dalla letteratura precedente sul tema in questione, a seguito dell'utilizzo degli scenari previsti nell'ambito del quadro normativo di vigilanza prudenziale attualmente in vigore. Le banche commerciali italiane risultano, inoltre, particolarmente esposte agli scenari rappresentati dallo short rates shock down e dallo steepener data la tipica struttura per scadenza delle posizioni nette, ottenuta a seguito della distribuzione delle varie poste di bilancio nella matrice per scadenza e per data di riprezzamento, caratterizzata da posizioni nette negative nel medio termine da 1 a 5 anni, riconducibili, essenzialmente, alla elevata quota dei depositi a vista distribuiti in base alle indicazioni regolamentari nelle fasce temporali fino a 5 anni, e da posizioni nette positive nelle fasce a lungo termine oltre i 5 anni, attribuibili, invece, alle quote capitali dei mutui a tasso fisso e ai titoli a tasso fisso in scadenza in tale orizzonte temporale. Il basso livello dei tassi di interesse negli ultimi anni e la conseguente applicazione del vincolo di non negatività ha ridotto, tuttavia, il numero di banche esposte allo short rates shock down.

La trattazione, che presenta una ampia ed esaustiva analisi sia della dinamica del quadro regolamentare che delle varie metodologie di misurazione funzionale a rendere maggiormente fruibile il tema in questione ad una pluralità di interlocutori, è strutturata come segue: il paragrafo 2 fornisce l'evoluzione del quadro normativo di vigilanza prudenziale; il paragrafo 3 descrive i principali contributi della letteratura sul tema in questione; il paragrafo 4 descrive le metodologie di calcolo e le innovazioni regolamentari oggetto della presente analisi; il paragrafo 5 riporta le evidenze empiriche nel periodo $2006-2016$ e il paragrafo 6 conclude con le relative implicazioni di policy.

\section{L'evoluzione della disciplina di vigilanza prudenziale}

L'attenzione verso il rischio di tasso di interesse del portafoglio bancario ha assunto una certa rilevanza a seguito della crisi delle Savings and Loans americane degli anni ' $80^{5}$. Subito dopo la crisi, infatti, gli economisti della Federal Reserve System svilupparono il modello Economic Value Model (EVM), adottando per la misurazione di tale tipologia di rischio la prospettiva del valore economico ${ }^{6}$. Nel 2004 il Comitato di Basilea ha emanato la versione definitiva del documento tecnico intitolato "Principles for the management of the interest rate risk" contenente 15 principi per la misurazione e la gestione del rischio in questione e una metodologia di misurazione basata sulla stessa logica sottostante il modello sviluppato dalla Federal Reserve System. In particolare, la metodologia proposta dal Comitato di Basilea: i) prevede la ripartizione dei valori contabili delle attività e delle passività di bilancio sensibili ai tassi di interesse in fasce temporali a cui sono associati specifici

${ }_{6}^{5}$ Per un approfondimento sulla crisi delle Savings and Loans americane si rimanda a Federal Deposit Insurance Corporation (1997).

${ }^{6}$ Il modello è descritto in Houpt e Embersit (1991) e, successivamente, analizzato in Wright e Houpt (1996), Sierra e Yeager (2004) e Sierra (2009). 
coefficienti di duration; ii) prende in considerazione sia una variazione parallela dei tassi di interesse di $+/-200$ punti base, sia il $1^{\circ}$ e il $99^{\circ}$ percentile delle variazioni dei tassi di interesse su base annua registrate in un periodo di osservazione di 6 anni (nel seguito della trattazione indicati, rispettivamente, con "scenario parallelo" e "metodo dei percentili"); iii) calcola un indicatore di rischio dato dal rapporto tra la variazione di valore economico del portafoglio bancario, a seguito della scenario di variazione dei tassi di interesse ipotizzato, e i fondi propri; e, iv) stabilisce, infine, che le Autorità di vigilanza devono prestare particolare attenzione alle c.d. banche anomale che presentano un livello dell'indicatore di rischio superiore al $20 \%$.

Nell'Accordo sul Capitale del giugno 2006, il Comitato di Basilea, pur ritenendo il rischio di tasso di interesse del portafoglio bancario potenzialmente significativo e, quindi, tale da richiedere un'adeguata copertura patrimoniale, ha deciso di inserire il rischio in questione nel secondo pilastro non prevedendo, quindi, un apposito requisito di capitale minimo regolamentare. Le motivazioni alla base di questa scelta sono riconducibili ai commenti pervenuti dal settore bancario e alle ulteriori analisi svolte dallo stesso Comitato che hanno evidenziato la considerevole eterogeneità tra le banche attive a livello internazionale in relazione sia alla natura del rischio sottostante, sia ai relativi procedimenti di gestione e monitoraggio. Successivamente nel 2009 il Comitato di Basilea ha pubblicato il documento tecnico intitolato "Range of practices and issues in economic capital frameworks" in cui viene fornita una rassegna delle migliori prassi adottate dalle banche per il calcolo del capitale economico associato alle varie tipologie di rischi. In relazione al rischio di tasso di interesse del portafoglio bancario l'attenzione si è focalizzata, principalmente, sulla modellizzazione delle opzionalità implicite di alcune poste di bilancio e sui possibili sviluppi della metodologia basata sullo scenario parallelo di +/- 200 punti base.

Nell'ottobre del 2006, inoltre, il Comitato delle Autorità Europee di Vigilanza Bancaria ha emanato il documento tecnico intitolato "Technical aspects of the management of interest rate risk arising from non-trading activities under the supervisory review process" che fornisce delle linee guida indirizzate sia alle banche sia alle Autorità di vigilanza nazionali riguardanti, rispettivamente, l'implementazione del processo di determinazione di adeguatezza patrimoniale (Internal Capital Adequacy Assessment Process - ICAAP) e di revisione e valutazione prudenziale (Supervisory Review and Evaluation Process - SREP). La Banca d'Italia ha recepito le indicazioni fornite dal Comitato di Basilea e del Comitato delle Autorità Europee di Vigilanza Bancaria nelle Nuove disposizioni di vigilanza prudenziale per le banche emanate nel dicembre 2006, che, per quanto riguarda il rischio in esame, sono state, successivamente, oggetto di modifiche e ulteriori integrazioni con il $6^{\circ}$ aggiornamento del 27 dicembre 2010. Infine, nel dicembre 2013 la nuova Circolare 285 emanata dalla stessa Banca d'Italia, in sostituzione della Circolare 263, ha recepito e lasciato invariato il quadro regolamentare sul rischio di tasso di interesse del portafoglio bancario.

Nel maggio del 2015 l'Autorità Bancaria Europea ha aggiornato le linee guida emanate dal Comitato delle Autorità Europee di Vigilanza Bancaria nel 2006 mediante un nuovo documento tecnico intitolato "Guidelines on the management of interest rate risk arising from non-trading activities" e nel successivo mese di ottobre ha pubblicato specifici orientamenti sulla gestione del rischio in questione. Nel giugno dello stesso anno il Comitato di Basilea ha emanato un documento tecnico per la consultazione finalizzato a rivedere l'attuale quadro regolamentare sul rischio di tasso di interesse del portafoglio bancario. Il documento tecnico in questione prevedeva due possibili opzioni di riforma. La prima concernente il possibile inserimento del rischio di tasso di interesse del portafoglio bancario nell'ambito del I pilastro con un conseguente calcolo di un requisito minimo di capitale regolamentare mediante un approccio standardizzato. La seconda, invece, manteneva l'attuale collocazione nel II pilastro, con la relativa stima di capitale interno in sede ICAAP, prevedendo, tuttavia, un significativo rafforzamento del quadro normativo attualmente in vigore. L'eventuale collocazione nel I pilastro mirava a promuovere maggiore trasparenza e comparabilità tra gli intermediari al fine di garantire sia una più appropriata consapevolezza da parte degli operatori di mercato sull'adeguatezza patrimoniale delle banche sia un comune level playing field a livello internazionale. L'eventuale conferma, invece, nell'ambito del II pilastro si basava sulla necessità di garantire un maggiore adattamento alle differenti condizioni di mercato e alle prassi bancarie attualmente in essere tra le varie giurisdizioni ${ }^{7}$. Il

${ }^{7}$ La scelta tra I e II pilastro ha rilevanti implicazioni sia in una prospettiva micro, in termini di solidità della singola banca e di definizione delle appropriate strategie di $A L M$, sia in una prospettiva macro, con riferimento alla stabilità globale del sistema e all'offerta di credito all'economia. Come affermato da AIFIRM (2015), che ha partecipato al processo di consultazione mediante la predisposizione di uno specifico Position Paper, l'adozione di un approccio di I pilastro comporta necessariamente una standardizzazione delle metodologie che conduce ad una minore precisione nella stima dell'esposizione al rischio e comprensione delle determinanti della stessa. Vi è, quindi, una maggiore probabilità che la banca determini un ammontare di capitale interno che sottostima o sovrastima l'effettiva esposizione al rischio. $\mathrm{Nel}$ primo caso errori nella stima possono rappresentare una minaccia per la stabilità della singola istituzione creditizia e, per tale via, del sistema bancario globale; nel secondo caso, invece, un eventuale sovrastima condurrebbe ad una riduzione della potenziale offerta di credito all'economia, che, come noto, è funzione del capitale libero a disposizione delle banche dato dalla differenza tra l'ammontare dei fondi propri e il totale del capitale interno posto a presidio delle varie tipologie di rischio. La standardizzazione delle metodologie può fornire anche indicazioni distorte per l'individuazione delle strategie di $A L M$ funzionali al raggiungimento degli obiettivi strategici declinati nel piano industriale e influenzare, in tal modo, l'esposizione al rischio e la performance delle stesse banche. Da un lato, infatti, una sottostima del capitale interno potrebbe condurre i manager ad assumere maggiore rischio; dall'altro lato una sovrastima potrebbe, invece, disincentivare le banche ad adottare strategie che conducono a una maggiore redditività. La scelta di collocare tale tipologia di rischio nell'ambito del II pilastro è stata, quindi, ritenuta da AIFIRM (2015) la soluzione più appropriata data la possibilità per le banche di adottare, in tale contesto, i propri modelli interni. Rispetto ad un approccio di I pilastro, un approccio di II pilastro, infatti, consente: i) una 
documento tecnico pubblicato a seguito della suddetta consultazione nell'aprile del 2016 ha confermato la collocazione del rischio in questione nell'ambito del secondo pilastro di Basilea data la natura eterogenea dello stesso rafforzandone, tuttavia, il quadro normativo di riferimento. Nello specifico il nuovo framework regolamentare delineato dal Comitato di Basilea oltre all'aggiornamento dei relativi principi prevede: i) l'introduzione di sei specifici scenari di variazione su base annua dei tassi di interesse (parallel shock up, parallel shock down, short rates shock up, short rates shock down, steepener shock e flattener shock) con cui valutare l'esposizione al rischio nella prospettiva del valore economico; ii) la misurazione, a fini della stima della complessiva esposizione al rischio, anche dell'impatto sul margine di interesse e/o sugli utili attesi mediante l'applicazione del solo scenario parallelo; iii) specifiche indicazioni metodologiche circa la modellizzazione delle poste di bilancio caratterizzate da elementi di opzionalità; iv) l'introduzione un nuovo framework matematico nell'ambito dell'approccio del valore economico basato sul regime finanziario della capitalizzazione continua; v) la definizione, in maniera dettagliata, delle informazioni da fornire al mercato nell'ambito dell'attività di disclosure; e, infine, iv) la revisione della soglia di attenzione utilizzata ai fini dell'individuazione delle banche anomale dal $20 \%$ al $15 \%$.

Il 26 novembre 2016 la Commissione Europea ha presentato un pacchetto globale di riforme per rafforzare ulteriormente la resilienza delle banche dell'Unione Europea. Le proposte di modifica dell'attuale quadro normativo di vigilanza prudenziale riguardano, con riferimento al rischio di tasso di interesse del portafoglio bancario, l'articolo 84 e il paragrafo 5 dell'art.98 della Direttiva 2013/36/UE e l'art.448 del Regolamento (UE) 575/2013. Il nuovo art.84 della Direttiva 2013/36/UE richiede, al paragrafo 1, alle banche l'applicazione di sistemi interni o della metodologia standardizzata per identificare, valutare, gestire e attenuare i rischi derivanti da variazioni potenziali dei tassi di interesse che influiscono non solo il valore economico del capitale proprio ma anche il margine di interesse. Lo stesso articolo, al paragrafo 3, sottolinea che le autorità di vigilanza competenti possono chiedere alle banche di utilizzare la metodologia standardizzata qualora i modelli interni, di cui al paragrafo 1, non siano ritenuti soddisfacenti. Il paragrafo 2 richiede, inoltre, alle banche anche la valutazione e il monitoraggio nella prospettiva sia del valore economico dei fondi propri che del margine di interesse della componente legata a variazioni potenziali dei differenziali creditizi. Infine, i successivi paragrafi 4 e 5 incaricano l'Autorità Bancaria Europea di elaborare, rispettivamente, norme tecniche di regolamentazione per specificare i dettagli della metodologia standardizzata e orientamenti volti a declinare i criteri da adottare ai fini dell'attuazione complessiva di quanto richiesto dallo stesso art.84.

Il nuovo paragrafo 5 dell'art.98 della Direttiva 2013/36/EU modifica, invece, i criteri di individuazione delle banche anomale, per le quali è richiesta l'adozione di adeguate misure di vigilanza, nell'ambito dell'approccio del valore economico riducendo la soglia di attenzione dal $20 \%$ al $15 \%$ e richiedendo di applicare, a tal fine, sei scenari di variazione dei tassi di interesse. Lo stesso art.98 al nuovo paragrafo 5 bis incarica l'Autorità Bancaria Europea di elaborare norme tecniche di regolamentazione per specificare: i) i sei scenari prudenziali di shock da applicare ai tassi di interesse per ciascuna valuta; ii) ipotesi di modellizzazione e parametriche comuni ai fini del calcolo dell'impatto del valore economico del capitale proprio; e c) se richiesta anche l'adozione di misure di vigilanza in caso di diminuzione del margine di interesse a seguito dell'applicazione dei differenti scenari di variazione dei tassi di interesse. Il nuovo art.448 del Regolamento 575/2013 disciplina, infine, con maggiore dettaglio le informazioni quantitative e qualitative sul rischio di tasso di interesse del portafoglio bancario sulla base di quanto disposto ai sopra descritti art.84 e paragrafo 5 dell'art.98 della Direttiva 2013/36/UE. È interessante sottolineare che tale articolo dispone esplicitamente che i sei scenari devono essere utilizzati non solo nell'ambito dell'approccio del valore economico ma anche in quello del margine di interesse a differenza di quanto stabilito dal Comitato di Basilea secondo cui nell'approccio del margine di interesse si applica solo lo scenario parallelo dei $+/-200$ punti base.

Nel febbraio 2017 la Banca Centrale Europea ha avviato uno stress test sul rischio di tasso di interesse del portafoglio bancario su un campione di 110 banche significative basato sui dati di bilancio al 31/12/2016 e relativo all'orizzonte temporale 2017-2019. Lo stress test ha riguardato sia l'impatto sul valore economico dei fondi propri sia sul margine di interesse mediante l'applicazione di 5 differenti scenari di variazione dei tassi di interesse da applicare allo scenario base di cui al 31/12/2016. Allo scenario parallelo dei +/-200 punti base sono stati aggiunti i due nuovi scenari steepener e flattener introdotti dal Comitato di Basilea e un ulteriore scenario di variazione, detto end-2010, che riconduceva il livello dei tassi di interesse osservato al 31/12/2016 ad una situazione precedente la crisi del debito sovrano e nello specifico alla data del 31/12/2010. Il documento tecnico redatto dalla BCE ai fini dell'implementazione dello stress test intitolato " $E C B$ Sensitivity analysis of IRRBB - Stress test 2017" riporta i relativi dettagli metodologici tra cui di particolare interesse è la non applicazione per specifiche poste di bilancio del vincolo di non negatività a seguito dell'adozione dei differenti scenari di variazione dei tassi di interesse ipotizzati. Gli esiti dello stress test pubblicati nel successivo mese di ottobre evidenziano un impatto piuttosto limitato, in media, dei vari scenari nell'ambito dell'approccio del valore economico con posizioni alquanto

maggiore precisione nella stima del capitale interno da porre a presidio del rischio in questione, ii) una più accurata comprensione delle determinanti della natura e della tipologia dell'esposizione al rischio e iii) una più appropriata selezione delle strategie di $A L M$. 
eterogenee tra le banche. L'applicazione dello scenario parallelo verso l'alto risulta, in media, il più penalizzante con un indicatore di rischio, calcolato sulla base del capitale primario di classe 1 , del 2,7\%. Lo scenario end-2010 e il flattener conducono ad un indicatore di rischio pari, rispettivamente, al 2,3\% e all'1,4\%. I restanti scenari rappresentati dallo spostamento parallelo verso il basso e dallo steepener fanno, invece, registrare un incremento del valore economico del capitale primario di classe 1 pari, rispettivamente, allo $0,9 \%$ e allo $0,4 \%$.

Nello stesso mese di ottobre 2017 l'Autorità Bancaria Europea ha emanato un documento di consultazione finalizzato all'aggiornamento delle linee guida pubblicate nel maggio del 2015. Il documento in consultazione recepisce i sei scenari di variazione dei tassi di interesse proposti dal Comitato di Basilea e introduce una nuova soglia di attenzione sui predetti scenari pari al $15 \%$ del capitale primario di classe 1 in aggiunta a quella sullo scenario dei $+/-200$ punti base pari al $20 \%$ dei fondi propri. Inoltre, tra le varie tematiche oggetto di consultazione si segnala: i) l'utilizzo dell'approccio del margine di interesse o degli utili attesi in aggiunta a quello già utilizzato del valore economico ai fini della misurazione della complessiva esposizione al rischio; e ii) la possibilità di applicare floor inferiori allo zero e, quindi, derogare al vincolo di non negatività per specifiche poste di bilancio partendo da un livello di -150 punti base sulla fascia a vista con un incremento di 5 punti base per anno fino a raggiungere un livello pari a 0 per scadenze uguali e superiori ai 30 anni.

Infine, il $20^{\circ}$ aggiornamento della Circolare 285/2013 della Banca d'Italia pubblicato lo scorso novembre 2017 integra le disposizioni sul rischio di tasso di interesse del portafoglio bancario per recepire gli Orientamenti dell'Autorità Bancaria Europea emanati nell'ottobre del 2015. Nello specifico, in base alle modifiche apportate, le banche devono valutare la propria esposizione al rischio sia in termini di variazione del valore economico (unico approccio finora previsto dalle disposizioni di vigilanza) sia in termini di variazione del margine di interesse o degli utili attesi, considerando anche spostamenti non paralleli della curva dei rendimenti. Inoltre, nel calcolo dell'indicatore di rischio regolamentare le banche devono conformarsi ai criteri e ai vincoli previsti dagli Orientamenti che prevedono, in particolare, l'utilizzo di una durata media di riprezzamento dei depositi a vista non superiore ai 5 anni. È prevista, inoltre, la possibilità di escludere dal calcolo i contratti di opzione se incorporati in altre poste di bilancio ${ }^{8}$. Infine, nella conduzione delle prove di stress, in conformità del principio di proporzionalità richiamato anche dagli stessi Orientamenti, le banche appartenenti alla classe 1 considerano anche i seguenti aspetti: a) i cambiamenti sostanziali delle relazioni tra i principali tassi di mercato (rischio base); e b) i cambiamenti delle ipotesi formulate con riferimento al comportamento dei clienti rispetto ai depositi a vista e alle altre opzioni comportamentali incorporate in attività e passività come, ad esempio, le opzioni di rimborso anticipato dei prestiti a favore della clientela. Le banche appartenenti alla classe 2 considerano, invece, variazioni nelle principali ipotesi riferite a modelli comportamentali della clientela e, solo se rilevante, il rischio base.

\section{Una rassegna della letteratura}

Il presente paragrafo fornisce una breve sintesi dei contributi che hanno analizzato la robustezza del framework metodologico proposto dal Comitato di Basilea nel 2004 e, successivamente, recepito dalla Banca d'Italia prima nella Circolare 263 del dicembre 2010 e, successivamente, nella Circolare 285 nel dicembre 2013. Al riguardo si segnala, innanzitutto, il contributo di Entrop et al. (2009) che analizza l'insieme delle ipotesi sottostanti il modello regolamentare proposto dal Comitato di Basilea ${ }^{9}$. Nello specifico, gli autori analizzano come varia l'esposizione al rischio di una banca se

${ }^{8}$ Nello specifico, nell'ambito della classificazione delle poste di bilancio nelle varie fasce temporali della matrice per scadenza e per data di riprezzamento i contratti di opzione a favore della banca, se incorporati in altre poste di bilancio (ad esempio clausole di floor presenti in attività a tasso variabile o clausole di cap presenti in passività a tasso variabile) possono essere esclusi. In tal caso, le banche assicurano un trattamento di tali opzioni che sia omogeneo nell'ambito dello stesso processo ICAAP e, di norma, coerente nel tempo, fornendo nel resoconto sul processo ICAAP informazioni sul trattamento prescelto e su eventuali modifiche rispetto all'anno precedente.

${ }^{9}$ In linea con tale contributo è l'analisi effettuata da Curcio e Gianfrancesco (2011) pubblicata sulla Newsletter AIFIRM. Nello specifico, gli autori testano la robustezza delle metodologie regolamentari calcolando il livello dell'indicatore di rischio in corrispondenza: a) della scadenza mediana e degli estremi di ciascuna delle 14 fasce temporali della matrice per scadenza e per data di riprezzamento; b) della duration modificata approssimata fornita dalle Autorità di vigilanza e di un indicatore di key rate duration, calcolato sulla base della struttura per scadenza dei tassi di interesse vigente al momento della valutazione; c) della distribuzione delle poste a vista effettuata con i criteri regolamentari in vigore prima e dopo il 2010. Prima delle modifiche apportate dal $6^{\circ}$ aggiornamento del 27 dicembre 2010 della Circolare 263 i depositi a vista erano allocati nelle varie fasce temporali della matrice per scadenza e per data di riprezzamento secondo il seguente criterio: nella fascia temporale a vista per un ammontare pari a quello dei conti correnti attivi. Il rimanente importo era allocato nelle successive quattro fasce temporali (da fino a 1 mese a da oltre 6 mesi a 1 anno) in misura proporzionale al numero dei mesi in esse contenuto. Le modifiche apportate dal suddetto aggiornamento della Circolare 263 prevedono, invece, l'allocazione dei depositi a vista per il $25 \%$ del loro ammontare nella fascia temporale a vista e la rimanente parte nelle successive otto fasce temporali (da fino a 1 mese a da oltre 4 anni a 5 anni) in proporzione del numero di mesi in esse contenuti. Le evidenze empiriche ottenute su una ipotetica banca, determinata aggregando i dati relativi a 15 banche commerciali italiane di piccola e media dimensione al 31/12/2009, supportano la tesi che le differenti ipotesi relative alla distribuzione delle poste a vista impattano in maniera più significativa sull'indicatore di rischio rispetto a quelle relative alla collocazione delle poste di bilancio all'interno delle fasce temporali e al coefficiente di duration adottato. La differente collocazione di tale aggregato di bilancio può, in alcuni casi, condurre al fenomeno della c.d. inversione dell'esposizione al rischio, vale a dire di banche esposte a variazioni in aumento dei tassi di interesse con il vecchio criterio di allocazione che a seguito dell'adozione del nuovo criterio di allocazione diventano esposte a variazioni in diminuzione dei tassi di interesse. 
sono modificate le ipotesi riguardanti : i) la collocazione dell'ammontare dei depositi a vista in un orizzonte temporale che va dalla scadenza a vista fino a quella a 5 anni; ii) la collocazione delle poste di bilancio all'interno delle fasce temporali della matrice per scadenza; iii) la numerosità e, di conseguenza, dall'ampiezza delle varie fasce temporali; iv) le modalità di ammortamento delle varie poste di bilancio; e, infine, v) lo spread tra il tasso contrattuale e il tasso di mercato utilizzato ai fini del calcolo della duration associata a ciascuna fascia temporale. Le evidenze empiriche riportate, ottenute mediante l'applicazione dello scenario parallelo dei +200 punti base al sistema bancario tedesco nel suo complesso, supportano la tesi secondo cui il livello dell'indicatore di rischio dipende in maniera rilevante dalle ipotesi sottostanti. La metodologia di misurazione proposta dal Comitato di Basilea dovrebbe, quindi, essere utilizzata, secondo gli stessi autori, con cautela a fini sia di vigilanza prudenziale nell'ambito della individuazione delle banche anomale, sia di misurazione e gestione del rischio da parte delle singole banche.

Altri contributi analizzano nello specifico le implicazioni sull'esposizione al rischio derivanti dai criteri di allocazione delle poste di bilancio nelle varie fasce temporali della matrice per scadenza e per data di riprezzamento. In tale ambito si segnala Entrop et al. (2008), che sviluppano un modello, detto TAM (Time Series Accountig Based Model), in grado di determinare la struttura per scadenza dei flussi di cassa associati alle attività e alle passività di bilancio sulla base delle serie storiche dei dati provenienti da differenti fonti informative. La metodologia sviluppata dagli autori non si basa, quindi, su dati puntuali come, invece, quella proposta dal Comitato di Basilea. Ciò consente di stimare con maggiore precisione la distribuzione delle poste di bilancio rispetto all'ipotesi di concentrazione delle stesse nel punto medio delle varie fasce temporali della matrice per scadenza e per data di riprezzamento regolamentare. Le evidenze empiriche relative all'applicazione dello scenario parallelo dei $+/-200$ punti base ottenute su un campione di banche tedesche al dicembre del 2005, mediante l'utilizzo di dati con frequenza mensile disponibili a partire dal gennaio 1999, mostrano che le stime dell'esposizione al rischio ottenute mediante l'applicazione del TAM sono maggiormente in linea, rispetto a quelle ottenute mediante la metodologia proposta dal Comitato di Basilea, con quelle rilevate da un sistema di monitoraggio posto in essere dall'Autorità di vigilanza nazionale, che raccoglie le stime relative all'esposizione al rischio calcolate dalle banche sulla base dei modelli di misurazione interni.

L'allocazione delle poste di bilancio, ed in particolare quella dei depositi a vista, è stato oggetto di analisi da parte di Cocozza et al. (2015). Gli autori sviluppano un modello che consente di allocare i depositi a vista nelle varie fasce temporali della matrice per scadenza e per data di riprezzamento sulla base delle due opzionalità implicite in tali poste di bilancio rappresentate, da un lato, dalla facoltà della banca di modificare, in qualsiasi momento, il tasso contrattuale a seguito di variazioni dei tassi di mercato e, dall'altro lato, dalla facoltà concessa ai sottoscrittori di tali passività di prelevare, in qualsiasi momento, le somme depositate ${ }^{10}$. Gli autori, facendo riferimento ad un campione di 30 banche commerciali italiane al 31/12/2011, analizzano le implicazioni, data l'applicazione dello scenario parallelo dei +/-200 punti base, derivanti sia dal passaggio tra il vecchio e nuovo criterio regolamentare di allocazione dei depositi introdotto da Banca d'Italia nel 2010, sia dal nuovo criterio regolamentare all'adozione del modello proposto basato sulle due suddette opzionalità implicite. Le evidenze empiriche ottenute confermano la rilevanza della modellizzazione di tali poste di bilancio nell'ambito della misurazione dell'esposizione al rischio di tasso di interesse del portafoglio bancario ${ }^{11}$. L'utilizzo di diversi criteri di allocazione può modificare, infatti, non solo il livello dell'indicatore di rischio regolamentare ma anche la tipologia di esposizione dando luogo al fenomeno della c.d. inversione dell'esposizione al rischio. Si tratta, nello specifico, di banche esposte a variazioni in aumento dei tassi di interesse che a seguito di un differente criterio di allocazione dei depositi a vista diventano esposte a variazioni in diminuzione dei tassi di interesse. Nell'ambito delle evidenze riportate gli autori segnalano, infine, la presenza del fenomeno della neutralità al rischio, cioè di banche caratterizzate da un incremento di valore economico dei fondi propri a prescindere dallo scenario di variazione dei tassi in aumento o in diminuzione applicato. Il fenomeno della neutralità, come sottolineato dagli stessi autori, è determinato per lo scenario parallelo dei +/-200 punti base dall'applicazione del vincolo di non negatività dato il basso livello dei tassi di interesse registrato alla data di valutazione.

\footnotetext{
${ }^{10}$ La prima opzionalità è modellizzata mediante un modello a correzione di errore, che consente di analizzare il meccanismo di trasmissione delle variazioni dei tassi di mercato sui tassi applicati dalle banche su tali poste di bilancio mediante la procedura proposta da Engle e Granger (1987). La seconda opzionalità è, invece, modellizzata mediante una metodologia basato sul concetto di Value at Risk (VaR) di tipo parametrico, che considera come fattore di rischio la componente ciclica della serie storica dei volumi dei depositi a vista attorno al relativo trend ottenuto mediante l'applicazione del filtro di Hodrick-Prescott (1997).

${ }^{11}$ Il tema della modellizzazione delle poste a vista è stato analizzato, con specifico riferimento al meccanismo di trasmissione della politica monetaria sui tassi bancari, anche nel contributo di Gianfrancesco e Giliberto (2014) pubblicato sulla Newsletter AIFIRM. Gli autori focalizzano, in particolare, l'attenzione sulle implicazioni derivanti dalle tensioni registrate sui mercati finanziari a seguito della crisi dei mutui sub-prime e del debito sovrano. Le evidenze empiriche ottenute, nel periodo che va dal 1999 al 2013, mostrano: a) una diminuzione dell'entità complessiva della variazione del tasso di mercato trasmessa dalle banche sui tassi applicati sulle poste a vista; e b) un aumento del ritardo da parte delle banche nell'adeguamento dei tassi applicati su tale aggregato di bilancio a seguito di variazioni sia in aumento sia in diminuzione dei tassi di mercato. Gli autori mostrano, infine, che le suddette evidenze impattano in modo differente sull'esposizione al rischio di una banca, sia nell'approccio del valore economico che del margine di interesse, a seconda che la stessa banca sia esposta a variazioni in aumento o in diminuzione dei tassi di interesse.
} 
Ulteriori contributi focalizzano l'attenzione sullo sviluppo di metodologie più sofisticate di quelle regolamentari effettuando un confronto tra le differenti evidenze ottenute in termini di esposizione al rischio. In tale ambito rientra Fiori e Iannotti (2007), che propongono una metodologia di misurazione in grado di tener conto della curtosi e della asimmetria delle distribuzioni delle variazioni dei tassi di interesse mediante l'applicazione di una specifica tecnica di local smoothing. La metodologia proposta, basata sulle simulazioni Monte Carlo, consente di stimare, a partire dalle variazioni su base giornaliera dei tassi di interesse, le tre componenti principali della struttura per scadenza dei tassi di interesse rappresentative, rispettivamente, dello spostamento parallelo (shift), della variazione nella pendenza (tilt o rotation) e della modifica nella curvatura (twist). I coefficienti di sensibilità sono, inoltre, calibrati su dati di mercato e tengono conto non solo del concetto di duration ma anche di quello di convexity. Le evidenze empiriche ottenute, su un campione costituito dalle principali banche italiane in termini di totale attivo a settembre del 2013, mostrano che la metodologia proposta dal Comitato di Basilea basata sullo scenario parallelo dei $+/-200$ punti base fornisce una misura dell'esposizione al rischio coerente con quella delle metodologie proposte dagli autori se i coefficienti di duration forniti dallo stesso Comitato sono calcolati sulla base dati di mercato vigenti alla data di valutazione.

Inoltre, Abdymomunov e Gerlach (2014) definiscono, sulla base della modellistica proposta in Nelson e Siegel (1987), una metodologia che consente di ottenere una pluralità di scenari caratterizzati da una più ampia varietà di variazioni nel livello, nella forma e nella pendenza della struttura per scadenza dei tassi di interesse rispetto a quelli utilizzati dalla banche nell'ambito sia dei tradizionali modelli interni, sia di quelli proposti dalle Autorità di vigilanza e dalla letteratura accademica. Le evidenze empiriche, ottenute sui dati di una tipica banca di grandi dimensioni statunitense disponibili nel Federal Deposit Insurance Corportation (FICD) Call Report, mostrano che la metodologia proposta conduce, rispetto alle altre utilizzate nel confronto, ad una maggiore riduzione del valore economico dei fondi propri rilevandosi, quindi, di maggiore utilità in un contesto sia di stress test che di reverse stress test.

Infine, Cerrone et al. (2017) sviluppano, facendo riferimento al metodo proposto da Lopez (1999), una procedura di back-testing finalizzata a valutare se il capitale interno stimato ex-ante a presidio del rischio di tasso di interesse del portafoglio bancario, mediante differenti metodologie, rappresentate da quelle regolamentari attualmente in uso e da due più sofisticate basate su tecniche di simulazione storiche e Monte Carlo, è coerente con l'effettiva rischiosità della banca valutata ex-post sulla base delle effettiva dinamica dei tassi di interesse registrate nei successivi dodici mesi la data di valutazione. Le evidenze empiriche ottenute, su un campione di 130 banche commerciali italiane nel periodo 2006-2013, supportano la necessità di modificare il quadro normativo di vigilanza prudenziale attualmente in vigore. Le metodologie più sofisticate basate su tecniche di simulazioni storiche e Monte Carlo, infatti, forniscono una misura di capitale ex-ante maggiormente coerente con quella effettivamente richiesta ex-post rispetto alle metodologie regolamentari e non sono, inoltre, caratterizzate dal fenomeno della neutralità al rischio. Nell'ambito delle evidenze riportate gli autori segnalano la presenza del fenomeno della neutralità non solo nello scenario parallelo dei +/-200 punti base, così come evidenziato in Cocozza et al. (2015), ma anche in quello del metodo dei percentili. In relazione al metodo dei percentili, a differenza di quanto accade per lo scenario parallelo dei +/-200 punti base, l'applicazione del vincolo di non negatività non è, tuttavia, una condizione necessaria per il verificarsi del fenomeno della neutralità al rischio. In differenti date di valutazione, infatti, la dinamica dei tassi di interesse osservati negli ultimi 6 anni può, infatti, condurre, come effettivamente rilevato nel periodo oggetto di analisi , a particolari scenari associati al $1^{\circ}$ e al $99^{\circ}$ percentile delle distribuzioni delle variazioni su base annua dei tassi di interesse che, combinati con la struttura per scadenza delle posizioni nette generano il fenomeno della neutralità al rischio a prescindere dall'applicazione del vincolo di non negatività ${ }^{12}$.

\section{Le metodologie di misurazione}

Nel presente paragrafo sono descritte le metodologie di misurazione utilizzate ai fini della presente analisi. Nel dettaglio è, inizialmente, descritto l'attuale quadro normativo di vigilanza prudenziale basato sull'approccio del valore economico dei fondi propri cosi come declinato nel Titolo III, Capitolo 1 della Circolare 285/2013 della Banca d'Italia. Successivamente, sono descritti i sei nuovi scenari di variazione dei tassi di interesse proposti nel documento tecnico emanato dal Comitato di Basilea nel mese di aprile 2016.

\footnotetext{
${ }^{12}$ La questione della neutralità al rischio a seguito dell'applicazione delle metodologie regolamentari e, in generale, l'analisi delle differenti tipologie di esposizione al rischio è stata trattata anche nel contributo di Gianfrancesco (2016) pubblicato sulla Newsletter AIFIRM. L'autore analizza l'esposizione al rischio di tasso di interesse del portafoglio bancario di un campione di 40 banche commerciali italiane nel periodo 2006-2015 al fine di fornire le linee guida funzionali alla comprensione delle determinanti dell'esposizione al rischio in questione e la conseguente identificazione delle strategie di Asset \& Liability Management(ALM) più appropriate per il raggiungimento degli obiettivi di rischio definiti in sede di Risk Appetite Framework $(R A F)$. Nello specifico, il contributo focalizza l'attenzione sulle due metodologie regolamentari rappresentante dallo scenario parallelo dei $+/-200$ punti base e dal metodo dei percentili fornendo analisi e indicazioni riguardanti: a) le differenti tipologie di esposizione al rischio (banca esposta a variazioni in aumento dei tassi di interesse, in diminuzione dei tassi di interesse e neutrale al rischio); b) i differenti impatti del portafoglio titoli di proprietà in corrispondenza delle varie tipologie di
} 


\section{L'attuale quadro normativo di vigilanza prudenziale basato sull'approccio del valore economico}

La misurazione dell'esposizione al rischio di tasso di interesse del portafoglio bancario viene effettuata sulla base dell'algoritmo semplificato definito nel Titolo III, Capitolo 1, Allegato C della Circolare della Banca d'Italia n.285 del dicembre 2013. Il modello in questione richiede per ogni singola valuta rilevante ${ }^{13}$ e per l'aggregato delle valute non rilevanti il calcolo per ciascuna fascia temporale della matrice per scadenza e per data di riprezzamento di una posizione netta ottenuta sommando le attività e passività che scadono o si riprezzano nell'orizzonte temporale in questione. Specifici criteri sono definiti per particolari poste di bilancio tra cui i depositi a vista che sono allocati per il $25 \%$ nella fascia temporale a vista e la rimanente parte nelle varie fasce temporali che coprono un orizzonte di 5 anni in proporzione al numero di mesi in esse contenuti $^{14}$. Le posizioni nette cosi ottenute sono poi moltiplicate per un coefficiente di duration modificata approssimata e per la variazione ipotizzata dei tassi di interesse. In simboli:

dove:

$$
\Delta P N_{i}=P N_{i} D M_{i} \Delta r_{i} \quad \text { (1.) }
$$

- $\quad \mathrm{PN}_{\mathrm{i}}$ e $\Delta \mathrm{PN}_{\mathrm{i}}$ rappresentano, rispettivamente, la posizione netta e la posizione netta ponderata relative alla i-esima fascia temporale;

- $\quad \mathrm{DM}_{\mathrm{i}}$ è la duration modificata approssimata associata alla $i$-esima fascia temporale;

- $\Delta \mathrm{r}_{\mathrm{i}}$ è la variazione ipotizzata del tasso di interesse associata alla $i$-esima fascia temporale.

La (1.) è sostanzialmente analoga alla nota relazione che lega la variazione del prezzo di un titolo a tasso fisso e scadenza determinata alla variazione del suo tasso interno di rendimento a scadenza mediante la duration modificata del titolo stesso. L'unica differenza è che nella (1.) non compare il segno meno nel membro di destra dell'equazione. Di conseguenza, un valore positivo (negativo) di $\Delta \mathrm{PN}_{\mathrm{i}}$ corrisponde a una riduzione (aumento) di pari importo del valore economico della posizione netta $i$-esima. Per ciascuna valuta rilevante e per l'aggregato delle valute non rilevanti $(j=1, \ldots k)$ la somma delle posizioni nette ponderate ottenute in corrispondenza delle varie fasce temporali $(i=1, \ldots n)$ sono sommate tra loro se positive al fine di ottenere una stima della variazione del valore economico portafoglio bancario $(\Delta V E)$ a seguito dello scenario di variazione dei tassi di interesse ipotizzato. In simboli:

$$
\Delta V E=\sum_{j=1}^{k} \sum_{i=1}^{n} \Delta P N_{i j} \quad s e \sum_{i=1}^{n} \Delta P N_{i j}>0
$$

Il valore così ottenuto è rapportato al valore dei fondi propri (FP) al fine di ottenere un indicatore di rischio (IR) la cui soglia di attenzione è fissata, al $20 \%$. In simboli:

$$
I R=\frac{\Delta V E}{F P} \quad(3 .)
$$

In base a quanto disposto dalle Disposizioni di vigilanza prudenziale per le banche (Titolo III - Capitolo 1- Sezione II della Circolare n.285/2013 emanata dalla Banca d'Italia) tutte le banche, indipendentemente dalla classe di appartenenza, dalla metodologia utilizzata e dalle variazioni stimate/scenari prescelti per calcolare il capitale interno in condizioni ordinarie/di stress, valutano l'impatto di una variazione ipotetica dei tassi di interesse pari a +/- 200 punti base sull'esposizione al rischio di tasso di interesse del portafoglio bancario. Nel caso in cui si determini una riduzione del valore economico della banca superiore al 20\% dei fondi propri, la Banca d'Italia approfondisce con la specifica banca i risultati e si riserva di adottare gli opportuni interventi.

Le modifiche apportate nel dicembre 2010 introducono la possibilità di calcolare l'indicatore di rischio per la determinazione del capitale interno in condizioni di operatività ordinaria facendo riferimento alle variazioni annuali dei tassi di interesse rilevate in un periodo di osservazione di 6 anni, considerando, alternativamente, il $1^{\circ}$ (ribasso) o il 99 ${ }^{\circ}$ (rialzo) percentile. Nella stima del capitale interno in ipotesi di stress, invece, le variazioni ipotizzate dei tassi sono determinate sulla base di scenari prescelti dalla banca, oltre a quello della variazione di +/- 200 punti base. Gli scenari al ribasso, corrispondenti al $1^{\circ}$ percentile e allo scenario parallelo dei -200 punti base, devono, inoltre, essere corretti al fine di rispettare il vincolo di non negatività dei tassi di interesse in base al quale la variazione su base annua dei tassi di interesse ipotizzata non può

esposizioni al rischio di cui al precedente punto a); e, infine, c) le modalità di utilizzo dei derivati di copertura evidenziandone i differenti impatti a seconda delle varie tipologie di esposizione al rischio di cui al precedente punto a).

${ }^{13}$ In base al dettato normativo di vigilanza prudenziale si considerano rilevanti le valute il cui peso misurato come quota sul totale attivo oppure sul passivo del portafoglio bancario è superiore al $5 \%$. 
condurre la struttura vigente dei tassi di interesse alla data di valutazione ad assumere in uno o più nodi valori inferiori a zero $^{15}$.

Ai fini della stima dell'indicatore di rischio si utilizza una struttura per scadenza di 14 tassi di interesse (indicati nel proseguo della trattazione indicati anche con il termine key-rates) i cui nodi corrispondono alla scadenza a vista e ai punti medi delle altre 13 fasce temporali della matrice per scadenza e per data di riprezzamento fornita dalle Autorità di vigilanza. I key-rates utilizzati, in linea con le attuali prassi di settore, sono il tasso EONIA per la scadenza a vista, i tassi Euribor per le scadenze inferiori a 12 mesi e i tassi Swap per le scadenze uguali o superiori a 1 anno. In corrispondenza dei punti medi delle fasce temporali della matrice per scadenza e per data di riprezzamento per i quali non è disponibile un tasso di interesse di mercato il relativo key-rate è ottenuto, nella presente analisi, considerando quello associato all'estremo superiore della specifica fascia temporale ${ }^{16}$.

In linea con quanto disposto dal Comitato di Basilea nel documento tecnico del 2004 e dalla Banca d'Italia nella Circolare 285/2013 nell'ambito del metodo dei percentili si utilizza ai fini del calcolo delle variazioni su base annua dei keyrates la tecnica delle osservazioni sovrapposte. In base a tale tecnica le variazioni su base annua dei key-rates sono calcolate come differenza tra i livelli osservati in un dato anno e quelli rilevati nell'anno precedente. Utilizzando per ciascun nodo della struttura a termine dei key-rates una serie storica di sei anni di livelli su base giornaliera precedenti la data di valutazione si ottiene una serie storica di cinque anni di variazioni su base annua ${ }^{17}$. Le variazioni su base annua dei key-rates cosi ottenute sono, successivamente corrette per tener conto del vincolo di non negatività dei tassi di interesse.

Nello specifico il metodo dei percentili prevede la determinazione, in corrispondenza di ciascuna fascia temporale, del $1^{\circ}$ e del $99^{\circ}$ percentile della distribuzione storica delle variazioni su base annua del relativo key-rate. In tal modo si ottengono due differenti scenari. Il primo scenario composto per ciascuna fascia temporale da variazioni, in genere, negative dei key-rates; il secondo caratterizzato, invece, per ciascuna fascia temporale, da variazioni, in genere, positive. Ai fini della stima dell'indicatore di rischio i due scenari sopra definiti sono applicati alle posizioni nette ottenute sulla base dei dati di bilancio relativi alla data di valutazione mediante la (1.). Per ciascun scenario le posizioni nette ponderate così ottenute sono sommate tra loro mediante la (2.) in modo da ottenere una stima della variazione del valore economico del portafoglio bancario, che, rapportata ai fondi propri alla data di valutazione, consente di ottenere, sulla base della (3.), l'indicatore di rischio regolamentare.

\section{I sei scenari di variazione dei tassi di interesse proposti dal Comitato di Basilea}

I sei scenari di variazione dei tassi di interesse proposti nel documento tecnico del Comitato di Basilea pubblicato nell'aprile 2016 sono di seguito indicati:

i) parallel shock up: spostamento parallelo verso l'alto;

ii) parallel shock down: spostamento parallelo verso il basso;

iii) short rates shock up: spostamento verso l'alto di tutti i tassi di interesse con maggior ampiezza su quelli a breve termine;

\footnotetext{
${ }^{14}$ Per un maggiore dettaglio sui criteri di allocazione delle varie poste di bilancio nelle fasce temporali della matrice regolamentare si rimanda alla Circolare 272/2008 emanata dalla Banca d'Italia.

${ }^{15}$ Nell'ipotesi di applicazione di uno scenario caratterizzato da variazioni negative dei tassi di interesse in una o più fasce temporali della matrice per scadenza e per data di riprezzamento, se il livello del tasso di interesse vigente alla data di valutazione, in corrispondenza di una specifica fascia temporale, è positivo e inferiore, in valore assoluto, alla variazione negativa da applicare, si considera una variazione negativa di ampiezza uguale al livello del tasso di interesse vigente alla data di valutazione, in modo che quest'ultimo, a seguito dell'applicazione dello scenario utilizzato per la stima dell'esposizione al rischio, assuma valori non inferiori a zero.

$\mathrm{Se}$, invece, il livello del tasso di interesse riferito ad una specifica fascia temporale e vigente alla data di valutazione è negativo, si applica, in base alle recenti indicazioni fornite dall'EBA in una recente Frequenlty Asked Question (FAQ) del luglio 2017 (Question ID 2017_3121), una variazione pari a zero. Dalla $F A Q$, infatti, si deduce che il floor dello $0 \%$ si applica solo in corrispondenza di scenari caratterizzati da variazioni negative in corrispondenza delle fasce temporali dove, alla data di valutazione, si registrano livelli positivi dei tassi di interesse. Prima delle indicazioni dell'EBA la prassi di settore considerava, in genere, una variazione positiva di ampiezza pari al livello negativo del tasso di interesse vigente alla data di valutazione e tale da ridurre quest'ultimo a zero.

${ }^{16}$ Per la fascia temporale oltre i 20 anni è utilizzato il tasso swap a 25 anni.

${ }^{17}$ Nel dettaglio, per ogni fascia temporale la serie storica delle variazioni su base annua del relativo key-rate è stata calcolata partendo dalla differenza dei livelli registrati, rispettivamente, nell'ultimo giorno dell'anno corrispondente alla data di valutazione e nell'ultimo giorno dell'anno precedente. Si tratta, ovviamente, dei giorni in cui sono disponibili quotazioni di mercato. Successivamente si procede a ritroso calcolando la differenza tra il penultimo giorno disponibile dell'anno di valutazione e quello dell'anno precedente e così via fino a coprire i sei anni di livelli richiesti dalla normativa di vigilanza prudenziale. L'orizzonte temporale di un anno a cui fare riferimento per la stima dell'esposizione al rischio di tasso di interesse del portafoglio bancario è specificatamente indicato dal Comitato di Basilea nel documento tecnico del 2004 in quanto ritenuto coerente con la capacità degli intermediari di ristrutturare o coprire le esposizioni al fine di mitigare perdite generate da una eccezionale volatilità dei tassi di interesse. Lo stesso documento tecnico richiede l'utilizzo di un periodo storico di 5 anni di variazioni su base annua dei tassi di interesse, in quanto ritenuto abbastanza lungo da comprendere i recenti e rilevanti andamenti ciclici dei tassi di interesse. Tale periodo è considerato adeguato anche per alimentare i modelli interni di misurazione basati sul concetto di VaR con un intervallo di confidenza del $99 \%$.
} 
iv) short rates shock down: spostamento verso il basso di tutti i tassi di interesse con maggiore ampiezza su quelli a breve termine;

v) steepener shock (short rates down e long rates up): riduzione dei tassi di interesse a breve e medio termine e incremento di quelli a lungo termine;

vi) flattener shock (short rates up e long rates down): incremento dei tassi di interesse a breve e medio termine e riduzione di quelli a lungo termine

La calibrazione di tali scenari si basa sull'applicazione delle formule da (4.) a (8.) di seguito riportate, che consentono di determinare l'ampiezza e il segno delle variazioni su tutte le fasce temporali della matrice per scadenza e per data di riprezzamento.

$$
\Delta \mathrm{R}_{\text {parallel } \mathrm{c}}\left(\mathrm{t}_{\mathrm{k}}\right)= \pm \mathrm{R}_{\text {parallel } \mathrm{c}}
$$

$$
\begin{aligned}
& \Delta R_{\text {short }, e}\left(t_{k}\right)= \pm R_{\text {short }, e} * S_{\text {short }}\left(t_{k}\right)= \pm R_{\text {short }, e} * e^{\frac{-t_{k}}{x}} \\
& \Delta R_{\text {long }, e_{i}}\left(t_{k}\right)= \pm R_{\text {long, }_{e}} * S_{\text {long }}\left(t_{k}\right)= \pm R_{\text {long,ce }} *\left(1-e^{\frac{-t_{k}}{x}}\right)
\end{aligned}
$$

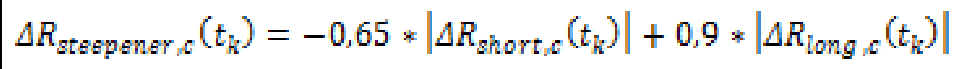

$$
\begin{aligned}
& \Delta R_{\text {flattener, }_{i}}\left(t_{k}\right)=+0,8 *\left|\Delta R_{\text {short }}\left(t_{k}\right)\right|-0,6 *\left|\Delta R_{\text {long,e }}\left(t_{k}\right)\right|
\end{aligned}
$$

Le suddette formule sono alimentate da specifici parametri, indicati con $\mathrm{R}_{\text {shocktype, }}$ riportati nella seguente Tabella 1 , che variano a seconda della tipologia di shock (parallelo, a breve termine e a lungo termine) e della valutazione di denominazione ${ }^{18}$.

Tabella 1. Parametri per la definizione dell'ampiezza dello shock

\begin{tabular}{cccccccc|}
\hline & AUD & CAD & CHF & EUR & GBP & JPY & USD \\
\hline Parallel & 300 & 200 & 100 & 200 & 250 & 100 & 200 \\
Short & 450 & 300 & 150 & 250 & 300 & 100 & 300 \\
Long & 200 & 150 & 100 & 100 & 150 & 100 & 150 \\
\hline
\end{tabular}

Fonte: Basel Committee on Banking Supervision (2016).

La definizione dello scenario parallelo verso l'alto e verso il basso di cui ai precedenti punti i) e ii) richiede in base alla (4.) l'applicazione del solo parametro definito nella precedente Tabella 1. Per la valuta di denominazione euro si applica, quindi, uno spostamento verso l'alto e verso il basso di +200 punti base su tutte le fasce temporali della matrice per scadenza e per data di riprezzamento, di 250 punti base per la sterlina inglese e così via. Per la valuta di denominazione euro vi è, quindi, coincidenza tra il nuovo shock parallelo proposto dal Comitato di Basilea e quello in vigore nell'attuale quadro normativo di vigilanza prudenziale. La definizione dello scenario basato sullo shock sul breve termine di cui ai precedenti punti iii) e iv) richiede l'applicazione della formula $\left(5\right.$.) che utilizza un fattore scalare $\left[S_{\text {short }}\left(t_{k}\right)\right]$ in grado di distribuire l'entità dello shock in maniera via via decrescente sulle fasce temporali a partire da quelle a breve termine fino a quelle a lungo termine. Nello specifico, $\mathrm{t}_{\mathrm{k}}$ è il punto medio della fascia temporale e $\mathrm{x}$ è un parametro che misura la velocità del decadimento dello shock posto pari a 4 dal Comitato di Basilea.

Ai fini della determinazione degli scenari steepener e flattener di cui ai precedenti punti v) e vi) è introdotta la formula (6.) che consente di distribuire l'entità dello shock, sulla base della stessa logica sottostante la precedente (5.), ma con la differenza che in questo caso lo shock è distribuito in maniera via via decrescente sulle fasce temporali a partire da quelle a lungo termine fino a quelle a breve termine. I due scenari v) e vi) sono, quindi, calcolati come combinazione delle formule (5.) e (6.) sulla base di appositi pesi, cosi come definito nelle formule (7.) e (8.).

18 La determinazione di tali parametri è effettuata, per ciascuna valuta, mediante un processo costituito da tre differenti fasi: a) determinazione di un valore rappresentativo del livello medio della struttura per scadenza dei tassi di interesse calcolato come media dei tassi di interesse rilevati su base giornaliera in corrispondenza di specifici nodi (3 e 6 mesi, 1, 2, 5, 7, 10 15 e 20 anni) nel periodo intercorrente tra il $03 / 01 / 2000$ e il $31 / 12 / 2015$; b) prodotto tra il valore di cui al precedente punto a) e i tre baseline global parameter definiti in funzione della tipologia di shock (parallelo, a breve termine e a lungo termine); e c) successiva rettifica dei tre valori ottenuti al precedente punto b) mediante l'applicazione di specifici cap e floor al fine di evitare l'utilizzo di variazioni dei tassi di interesse sia in 
Ad esempio, ipotizziamo di voler calcolare l'ampiezza della variazione del tasso di interesse associato alla fascia temporale da oltre 3 a 4 anni. Il punto medio di tale fascia $\left(\mathrm{t}_{\mathrm{k}}\right)$ è pari a 3,5. Il fattore scalare $\left[\mathrm{S}_{\text {short }}\left(\mathrm{t}_{\mathrm{k}}\right)\right]$ è conseguentemente pari a 0,42 . Sulla base di tali dati è possibile calcolare mediante la (5.) la variazione del tasso di interesse associato alla specifica scadenza per gli scenari iii) e iv) nel modo seguente:

$$
\Delta R_{\text {short }} e\left(t_{k}\right)= \pm R_{\text {short }, e} * S_{\text {short }}\left(t_{k}\right)= \pm 250 * 0,42= \pm 1,04 \text { bp }
$$

La variazione del tasso di interesse associato alla fascia temporale da oltre 3 anni a 4 anni è quindi per lo short rates shock up e per lo short rates shock down pari, rispettivamente, a +/- 104 punti base. L'applicazione della (7.) e della (8.) consente, infine, di calcolare la relativa variazione de tasso di interesse per gli scenari v) e vi) nel modo seguente:

$$
\begin{aligned}
& \Delta R_{\text {stegpeney } e_{i}}\left(t_{k}\right)=-0,65 * 250 * 0,42+0,9 * 100 *(1-0,42)=-0,15 b p \\
& \Delta R_{\text {flattener }_{i}}\left(t_{k}\right)=+0,8 * 250 * 0,42-0,6 * 100 *(1-0,42)=+0,48 b p
\end{aligned}
$$

La variazione del tasso di interesse associato alla fascia temporale da oltre 3 anni a 4 anni è quindi per lo steepener $\mathrm{e}$ per il flattener pari, rispettivamente, a -15 e +48 punti base. La seguente Tabella 2. Riporta le variazioni dei tassi di interesse associate agli scenari short rates shock up (SRU), short rates shock down (SRD), steepener (STEEP) e flattener (FLAT) con riferimento alle 14 fasce temporali della matrice per scadenza e data di riprezzamento attualmente in vigore e utilizzata nel prosieguo dell'analisi.

Tabella 2. Scenari introdotti dal Comitato di Basilea (valori percentuali)

\begin{tabular}{|cccccccccc|}
\hline $\begin{array}{c}\text { Fasce } \\
\text { temporali }\end{array}$ & $\begin{array}{c}\text { Punto } \\
\text { medio (tk) }\end{array}$ & \multicolumn{2}{c}{ Fattore scalare } & \multicolumn{2}{c}{ Parametro } & \multicolumn{3}{c|}{ Shock } \\
\hline Short & Long & Short & Long & SRU & SRD & STEEP & FLAT \\
\hline A vista & 0,00 & 1,00 & 0,00 & 250 & 100 & 2,50 & $-2,50$ & $-1,63$ & 2,00 \\
fino a 1m & 0,04 & 0,99 & 0,01 & 250 & 100 & 2,47 & $-2,47$ & $-1,60$ & 1,97 \\
1m-3m & 0,17 & 0,96 & 0,04 & 250 & 100 & 2,40 & $-2,40$ & $-1,52$ & 1,89 \\
3m-6m & 0,38 & 0,91 & 0,09 & 250 & 100 & 2,28 & $-2,28$ & $-1,40$ & 1,77 \\
6m-1a & 0,75 & 0,83 & 0,17 & 250 & 100 & 2,07 & $-2,07$ & $-1,19$ & 1,56 \\
\hline 1a-2a & 1,50 & 0,69 & 0,31 & 250 & 100 & 1,72 & $-1,72$ & $-0,84$ & 1,19 \\
2a-3a & 2,50 & 0,54 & 0,46 & 250 & 100 & 1,34 & $-1,34$ & $-0,45$ & 0,79 \\
3a-4a & 3,50 & 0,42 & 0,58 & 250 & 100 & 1,04 & $-1,04$ & $-0,15$ & 0,48 \\
4a-5a & 4,50 & 0,32 & 0,68 & 250 & 100 & 0,81 & $-0,81$ & 0,08 & 0,24 \\
\hline 5a-7a & 6,00 & 0,22 & 0,78 & 250 & 100 & 0,56 & $-0,56$ & 0,34 & $-0,02$ \\
7a-10a & 8,50 & 0,12 & 0,88 & 250 & 100 & 0,30 & $-0,30$ & 0,60 & $-0,29$ \\
10a-15a & 12,50 & 0,04 & 0,96 & 250 & 100 & 0,11 & $-0,11$ & 0,79 & $-0,49$ \\
15a-20a & 17,50 & 0,01 & 0,99 & 250 & 100 & 0,03 & $-0,03$ & 0,87 & $-0,57$ \\
Oltre 20a & 25,00 & 0,00 & 1,00 & 250 & 100 & 0,00 & 0,00 & 0,90 & $-0,59$ \\
\hline
\end{tabular}

Fonte: elaborazioni dell'Autore sulla base di quanto riportato in Basel Committee on Banking Supervision (2016).

Le evidenze della Tabella 2 mostrano che gli scenari di variazione dei tassi di interesse short rates shock up e short rates shock down sono tra loro simmetrici e caratterizzati da variazioni dello stesso segno, rispettivamente, positive e negative, che diminuiscono via via in termini di ampiezza in corrispondenza delle varie fasce temporali da quella a vista fino a quella oltre i 20 anni. Nell'ultima fasce temporale si registra per entrambi una variazione pari a zero. Gli scenari di variazione dei tassi di interesse steepener e flattener sono, invece, caratterizzati da variazioni di differente segno sulle varie fasce temporali della matrice per scadenza e per data di riprezzamento . Il primo è caratterizzato da variazioni negative via via decrescenti in ampiezza dalla fascia temporale a vista fino a quella da 3 anni a 4 anni e da variazioni positive via via crescenti in ampiezza dalla fascia da 4 a 5 anni fino a quella oltre i 20 anni. Il secondo, invece, da variazioni positive via via decrescenti in ampiezza dalla fascia a vista fino a quella da 4 a 5 anni e da variazioni negative via via crescenti in ampiezza dalla fascia da 5 a 7 anni a oltre i 20 anni.

aumento che in diminuzione irrealistiche. Per un ulteriore approfondimento si rimanda al documento tecnico emanato dal Comitato di 


\section{Le evidenze nel periodo 2006-2016}

Il presente paragrafo riporta le principali evidenze relative all'impatto dei differenti scenari di variazione dei tassi di interesse descritti nel corso della trattazione su un campione di 136 banche commerciali italiane nel periodo 2006-2016 sulla base dei dati di bilancio pubblicati al 31/12 di ogni anno. I dati relativi alle banche analizzate nel campione sono stati ottenuti nella matrice per scadenza e data di riprezzamento pubblicata nel bilancio di esercizio di fine anno nella Parte E della nota integrativa intitolata "Informazioni sui rischi e sulle relative politiche di copertura". Per i dati relativi alla dotazione patrimoniale si è fatto riferimento, invece, alla parte E della nota integrativa intitolata "Informazioni sul patrimonio". Il campione è costituito da banche, principalmente di piccola e media dimensione rientranti nella classe 3 ai fini ICAAP, per le quali sono disponibili i dati nell'intero periodo di riferimento ${ }^{19}$. La seguente Tabella 3 riporta la composizione dei principali aggregati dell'attivo e del passivo di bilancio in percentuale del totale attività e passività per cassa comprensive del valore nominale delle posizioni fuori bilancio. Le quote percentuali sono calcolate per ogni anno come media semplice dei valori ottenuti sulle singole banche. L'analisi dei dati evidenzia, nel corso del periodo oggetto di analisi, dal lato dell'attivo un incremento del peso dei titoli, che è passato dal 14,07\% nel 2006 al 30,41\% nel 2016. Nello specifico, la componente a medio termine (da 1 a 5 anni) ha fatto registrare un aumento dal 3,26\% al 6,21\% e quella a lungo termine (oltre i 5 anni) dall' $1,28 \%$ al 5,71\%. Si registra, allo stesso tempo, una contestuale riduzione dei prestiti erogati passati dal 76,51\% al 62,08\%. Dal lato del passivo si segnala, invece, dal un lato un incremento della componente a vista da clientela al dettaglio dal $38,69 \%$ al $47,53 \%$ e della componente altra raccolta a medio termine (da 1 anno a 5 anni), al cui interno rientra parte della raccolta verso la BCE, dall' $1,51 \%$ al $7,69 \%$ e, dall'altro lato, una riduzione dei titoli obbligazionari emessi dal $28,36 \%$ al $17,00 \%$.

Tabella 3. Composizione attivo e passivo di bilancio del campione di banche (valori percentuali)

\begin{tabular}{|lccccccccccc|}
\hline & 2006 & 2007 & 2008 & 2009 & 2010 & 2011 & 2012 & 2013 & 2014 & 2015 & 2016 \\
\hline Titoli scadenza inf. a 1a & 9,53 & 9,07 & 11,54 & 12,00 & 11,36 & 10,72 & 12,11 & 14,56 & 19,00 & 19,04 & 18,49 \\
Titoli scadenza tra 1a e 5a & 3,26 & 2,46 & 1,20 & 1,79 & 1,75 & 2,70 & 6,22 & 7,31 & 6,44 & 6,03 & 6,21 \\
Titoli scadenza sup. ai 5a & 1,28 & 1,11 & 0,60 & 1,26 & 1,51 & 1,75 & 3,48 & 4,86 & 4,75 & 5,60 & 5,71 \\
Prestiti a vista & 45,78 & 48,14 & 46,76 & 41,19 & 38,08 & 37,01 & 37,77 & 35,13 & 32,19 & 30,49 & 29,09 \\
Prestiti scadenza inf. a 1a & 19,79 & 23,53 & 23,96 & 25,92 & 29,38 & 26,69 & 22,66 & 20,47 & 20,58 & 21,47 & 22,09 \\
Prestiti scadenza tra 1a e 5a & 5,71 & 3,53 & 3,89 & 5,54 & 4,86 & 6,41 & 5,60 & 6,26 & 5,84 & 6,95 & 7,10 \\
Prestiti scadenza sup. ai 5a & 5,23 & 3,30 & 4,51 & 5,55 & 3,35 & 4,34 & 2,88 & 2,74 & 2,79 & 3,10 & 3,80 \\
\hline Posizioni lunghe fuori bilancio & 9,43 & 8,88 & 7,54 & 6,76 & 9,70 & 10,39 & 9,28 & 8,67 & 8,42 & 7,32 & 7,51 \\
\hline Conti correnti passivi da clientela & 38,69 & 37,29 & 37,14 & 41,07 & 39,72 & 37,02 & 33,54 & 34,87 & 37,66 & 43,14 & 47,53 \\
Altre componenti a vista & 8,97 & 8,88 & 8,74 & 8,83 & 9,41 & 8,97 & 10,02 & 8,10 & 7,80 & 7,75 & 7,47 \\
Altra raccolta inf. a 1a & 12,15 & 10,17 & 7,87 & 4,83 & 5,69 & 8,66 & 15,39 & 18,52 & 17,77 & 16,13 & 12,26 \\
Altra raccolta tra 1a e 5a & 1,51 & 1,04 & 0,42 & 0,26 & 0,34 & 1,51 & 1,48 & 2,13 & 2,43 & 4,09 & 7,69 \\
Altra raccolta sup. ai 5a & 0,32 & 0,19 & 0,17 & 0,17 & 0,19 & 0,18 & 0,15 & 0,17 & 0,22 & 0,28 & 0,29 \\
Titoli di debito scadenza inf. a 1a & 20,57 & 25,34 & 29,52 & 28,40 & 21,54 & 19,51 & 15,58 & 14,02 & 12,04 & 10,02 & 8,46 \\
Titoli di debito scadenza tra 1a e 5a & 7,16 & 7,30 & 8,00 & 8,79 & 12,34 & 12,84 & 13,66 & 12,46 & 11,81 & 10,24 & 8,00 \\
Titoli di debito scadenza sup. ai 5a & 0,63 & 0,51 & 0,37 & 0,43 & 0,65 & 0,68 & 0,60 & 0,66 & 0,79 & 0,75 & 0,54 \\
\hline Posizioni lunghe fuori bilancio & 9,99 & 9,27 & 7,78 & 7,22 & 10,11 & 10,64 & 9,59 & 9,07 & 9,48 & 7,60 & 7,74 \\
\hline Fon & & & &
\end{tabular}

Fonte: elaborazioni dell'Autore sui dati di bilancio al 31/12 di ogni anno.

La stima dell'esposizione al rischio è effettuata facendo riferimento all'attuale matrice per scadenza e per data di riprezzamento utilizzata ai fini della stima dell'indicatore di rischio regolamentare e composta da 14 fasce temporali. Inoltre, per l'intero periodo di riferimento si utilizza il criterio di distribuzione dei depositi introdotto nel 2011 che prevede la ripartizione di tali poste di bilancio nelle fasce temporali fino a 5 anni così come descritto nel precedente paragrafo 4 .. La matrice per scadenza e per data di revisione riportata nei bilanci bancari presenta, tuttavia, un numero di fasce temporali inferiori a quelle di cui alla matrice regolamentare. I dati sono stati, quindi, redistribuiti nelle varie fasce temporali della matrice regolamentare in proporzione del numero di mesi contenuti nelle stesse. Ad esempio, i volumi contenuti nella fascia

Basilea nell'aprile 2016.

${ }^{19}$ La Circolare 262 del 22 dicembre 2005 che disciplina gli schemi e le regole di compilazione del bilancio bancario stabilisce che la tabella concernente la distribuzione delle poste di bilancio per data di scadenza e di riprezzamento può non essere redatta se nella nota integrativa viene fornita un'analisi di sensitività del rischio di tasso di interesse in base ai modelli interni o ad altre metodologie che soddisfi quanto richiesto in materia di IFRS7. Quando detto fa sì che nella costituzione del campione sono state escluse quelle banche che non hanno pubblicato la tabella in questione anche in un solo anno del periodo oggetto di analisi. Le banche che scelgono di inserire nella nota integrativa le proprie analisi di sensitività invece della matrice per scadenza e per data di riprezzamento sono, da quanto emerso nell'ambito della fase di raccolta di dati, in genere quelle di maggiori dimensioni. 
temporale da 1 mese a 3 mesi della matrice di bilancio sono stati redistribuiti per $1 / 3$ nella fascia temporale fino ad 1 mese e per il 2/3 nella fascia temporale da oltre 1 mese fino a 3 mesi e così via. Per quel che concerne i tassi di interesse utilizzati si fa riferimento, infine, a quanto detto nel precedente paragrafo 4. Nell'ambito della presente analisi è stata utilizzata una unica matrice per scadenza e per data di revisione comprendente l'intero portafoglio bancario denominato in euro. Se non disponibile si è fatto riferimento alla matrice per scadenza e per data di riprezzamento relativa alle sole esposizioni in euro. In entrambi i casi l'algoritmo di cui alla formula (2.) è stato applicato per una unica valuta rilevante di denominazione.

Le implicazioni derivanti dalla struttura per scadenza delle posizioni nette sull'esposizione al rischio di tasso di interesse del portafogli bancario

La successiva Tabella 4 riporta per l'intero periodo di riferimento 2006-2016 il peso delle posizioni nette sui fondi propri, in corrispondenza delle varie fasce temporali della matrice per scadenza e data di riprezzamento, relativo all'intero campione oggetto di analisi calcolati per ogni anno come media semplice dei valori ottenuti sulle singole banche.

Tabella 4. Struttura per scadenza delle posizioni nette

(valori percentuali)

\begin{tabular}{|ccccccccccccccc|}
\hline Anno & A vista & Fino a 1m & 1m-3m & 3m-6m & 6m-1a & 1a-2a & 2a-3a & 3a-4a & 4a-5a & 5a-7a & 7a-10a & 10a-15a & 15a-20a & Oltre 20a \\
\hline 2006 & 2,19 & $-0,10$ & $-0,20$ & $-0,48$ & $-0,31$ & $-0,40$ & $-0,40$ & $-0,40$ & $-0,40$ & 0,12 & 0,18 & 0,07 & 0,07 & 0,07 \\
2007 & 2,63 & $-0,05$ & $-0,09$ & $-0,68$ & $-0,15$ & $-0,44$ & $-0,44$ & $-0,44$ & $-0,44$ & 0,08 & 0,12 & 0,05 & 0,05 & 0,05 \\
2008 & 2,69 & 0,00 & 0,01 & $-0,45$ & $-0,27$ & $-0,51$ & $-0,51$ & $-0,51$ & $-0,51$ & 0,07 & 0,11 & 0,07 & 0,07 & 0,07 \\
2009 & 2,22 & 0,17 & 0,33 & $-0,35$ & $-0,29$ & $-0,55$ & $-0,55$ & $-0,55$ & $-0,55$ & 0,12 & 0,19 & 0,09 & 0,09 & 0,09 \\
2010 & 2,14 & 0,35 & 0,70 & $-0,21$ & $-0,33$ & $-0,63$ & $-0,63$ & $-0,63$ & $-0,63$ & 0,10 & 0,15 & 0,07 & 0,07 & 0,07 \\
2011 & 2,35 & 0,11 & 0,23 & $-0,35$ & $-0,22$ & $-0,61$ & $-0,61$ & $-0,61$ & $-0,61$ & 0,16 & 0,24 & 0,10 & 0,10 & 0,10 \\
2012 & 2,29 & 0,00 & 0,01 & $-0,03$ & $-0,38$ & $-0,51$ & $-0,51$ & $-0,51$ & $-0,51$ & 0,19 & 0,29 & 0,09 & 0,09 & 0,09 \\
2013 & 2,18 & $-0,12$ & $-0,25$ & 0,37 & $-0,43$ & $-0,53$ & $-0,53$ & $-0,53$ & $-0,53$ & 0,23 & 0,34 & 0,10 & 0,10 & 0,10 \\
2014 & 1,73 & $-0,06$ & $-0,11$ & 1,00 & $-0,34$ & $-0,63$ & $-0,63$ & $-0,63$ & $-0,63$ & 0,26 & 0,39 & 0,09 & 0,09 & 0,09 \\
2015 & 1,35 & $-0,16$ & $-0,32$ & 1,24 & $-0,48$ & $-0,73$ & $-0,73$ & $-0,73$ & $-0,73$ & 0,27 & 0,40 & 0,10 & 0,10 & 0,10 \\
2016 & 0,94 & 0,33 & 0,65 & 1,13 & $-0,53$ & $-0,88$ & $-0,88$ & $-0,88$ & $-0,88$ & 0,32 & 0,48 & 0,11 & 0,11 & 0,11 \\
\hline Media & 2,07 & 0,04 & 0,09 & 0,11 & $-0,34$ & $-0,58$ & $-0,58$ & $-0,58$ & $-0,58$ & 0,17 & 0,26 & 0,09 & 0,09 & 0,09 \\
\hline
\end{tabular}

Fonte: elaborazioni dell'Autore su dati di bilancio al 31/12 di ogni anno

È interessante osservare che i criteri di allocazione definiti dal quadro normativo di vigilanza prudenziale conducono, in genere, ad una struttura per scadenza delle posizione nette caratterizzata da posizioni nette negative nelle fasce a medio termine da 1 anno a 5 anni riconducibili, essenzialmente, al peso dei depositi a vista che, come noto, sono classificati in un orizzonte temporale di 5 anni e alle altre forme tecniche di raccolta a scadenza come i depositi vincolati e le obbligazioni emesse che hanno, in genere, scadenza nel medio termine e da posizioni nette positive nella fasce temporali a lungo termine oltre i 5 anni riconducibili alla scadenza delle quote capitali dei mutui a tasso fisso e dei titoli a tasso fisso iscritti nel portafoglio bancario in scadenza su tale orizzonte temporale. Le posizioni nette negative a seguito di un aumento (diminuzione) dei key-rates danno luogo a posizioni nette ponderate negative (positive) e quindi ad un incremento (riduzione) del valore economico dei fondi propri. Al contrario le posizioni nette positive a seguito di un aumento (diminuzione) dei keyrates danno luogo a posizione nette ponderate positive (negative) e quindi ad una riduzione (incremento) del valore economico dei fondi propri. Le posizioni nette ponderate positive e negative che si formano, invece, sulle fasce temporali a breve termine entro un anno non impattano in maniera significativa sulla stima dell'indicatore di rischio dati i bassi coefficienti di duration ad esse associati. In tali fasce temporali rientrano le poste di bilancio a tasso variabile, quelle a tasso fisso in scadenza entro l'anno e la parte dei depositi a vista distribuita in tale orizzonte temporale.

La variazione di valore economico dei fondi propri, che rappresenta il numeratore dell'indicatore di rischio regolamentare, dipende quindi: i) dall'entità e dal segno delle posizioni nette in corrispondenza di ogni fascia temporale; ii) dalla duration ad esse associate; e iii) dall'ampiezza e dal segno delle variazioni dei key-rates a seconda dello specifico scenario utilizzato. L'ammontare dei fondi propri influenza il calcolo dell'indicatore di rischio in quanto computato al denominatore dello stesso e, quindi, rileva ai fini della verifica del mantenimento di un'esposizione al rischio sotto la soglia di allerta fissata dalle Autorità di vigilanza.

\section{Il livello dei tassi di interesse e l'applicazione del vincolo di non negatività}

La successiva Tabella 5 riporta i livelli dei tassi di interesse al 31/12 nei vari anni oggetto di analisi. Le evidenze mostrano che la struttura per scadenza dei key-rates ha subito nel corso degli anni una tendenziale riduzione dei livelli in corrispondenza di tutti i nodi, ed in particolare su quelli a breve termine, che ha condotto ad una maggiore inclinazione della stessa. La progressiva riduzione dei livelli ha condotto all'applicazione del vincolo di non negatività sugli scenari di 
variazione in diminuzione dei tassi di interesse richiesti dal quadro normativo di vigilanza prudenziale. Ad esempio a partire dal 2009 si registrano sulle fasce temporali che vanno da quella a vista fino a quella da oltre 1 anno a 2 anni livelli inferiori ai 200 punti base che hanno condotto all'applicazione del vincolo di non negatività sullo scenario parallelo dei -200 punti base. A partire dallo stesso anno il vincolo di non negatività è stato applicato anche al metodo dei percentili, allo short rates shock down e allo steepener. Inoltre, negli ultimi due anni si registrano valori negativi dei key-rates sui nodi della curva a breve e medio termine. Ciò fa sì che in base all'applicazione del vincolo di non negatività su tali nodi l'utilizzo di scenari di variazione su base annua dei tassi di interesse negativi conduce a considerare variazioni pari a zero, così come specificato nelle recenti indicazioni fornite dall'Autorità Bancaria Europea riportate nella nota 15 del precedente paragrafo 4.

Tabella 5. Struttura per scadenza dei key-rates associati alle varie fasce temporali della matrice per scadenza e data di revisione al 31/12 di ogni anno

(valori percentuali)

\begin{tabular}{|lcccccccccccccc}
\hline $\begin{array}{c}\text { Data di } \\
\text { valutazione }\end{array}$ & A vista & $\begin{array}{c}\text { Fino a } \\
\mathbf{1 m}\end{array}$ & $\mathbf{1 m - 3 m}$ & $\mathbf{3 m - 6 m}$ & $\mathbf{6 m - 1 a}$ & $\mathbf{1 a - 2 a}$ & $\mathbf{2 a - 3 a}$ & $\mathbf{3 a - 4 a}$ & $\mathbf{4 a - 5 a}$ & $\mathbf{5 a - 7 a}$ & $\mathbf{7 a - 1 0 a}$ & $\mathbf{1 0 a - 1 5 a}$ & $\mathbf{1 5 a - 2 0 a}$ & $\begin{array}{c}\text { Oltre } \\
\mathbf{2 0 a}\end{array}$ \\
\hline $31 / 12 / 2006$ & 3,69 & 3,63 & 3,66 & 3,85 & 3,95 & 4,13 & 4,13 & 4,13 & 4,13 & 4,15 & 4,20 & 4,27 & 4,31 & 4,31 \\
\hline $31 / 12 / 2007$ & 3,92 & 4,29 & 4,49 & 4,71 & 4,73 & 4,55 & 4,53 & 4,53 & 4,56 & 4,61 & 4,72 & 4,86 & 4,91 & 4,91 \\
\hline $31 / 12 / 2008$ & 2,35 & 2,60 & 2,79 & 2,97 & 3,02 & 2,76 & 2,96 & 3,12 & 3,24 & 3,46 & 3,74 & 3,90 & 3,86 & 3,67 \\
\hline $31 / 12 / 2009$ & 0,41 & 0,45 & 0,56 & 0,99 & 1,13 & 1,86 & 2,26 & 2,56 & 2,81 & 3,21 & 3,60 & 3,96 & 4,07 & 4,02 \\
\hline $31 / 12 / 2010$ & 0,82 & 0,78 & 0,89 & 1,23 & 1,37 & 1,56 & 1,95 & 2,21 & 2,48 & 2,89 & 3,31 & 3,64 & 3,70 & 3,67 \\
\hline $31 / 12 / 2011$ & 0,63 & 1,02 & 1,18 & 1,62 & 1,79 & 1,32 & 1,38 & 1,53 & 1,73 & 2,07 & 2,37 & 2,67 & 2,69 & 2,62 \\
\hline $31 / 12 / 2012$ & 0,13 & 0,11 & 0,15 & 0,32 & 0,43 & 0,37 & 0,46 & 0,61 & 0,77 & 1,13 & 1,57 & 2,02 & 2,17 & 2,22 \\
\hline $31 / 12 / 2013$ & 0,45 & 0,22 & 0,26 & 0,39 & 0,48 & 0,54 & 0,77 & 1,00 & 1,26 & 1,68 & 2,16 & 2,59 & 2,71 & 2,74 \\
\hline $31 / 12 / 2014$ & 0,14 & 0,02 & 0,04 & 0,17 & 0,25 & 0,18 & 0,22 & 0,29 & 0,36 & 0,53 & 0,82 & 1,15 & 1,33 & 1,42 \\
\hline $31 / 12 / 2015$ & $-0,13$ & $-0,21$ & $-0,17$ & $-0,04$ & 0,00 & $-0,03$ & 0,06 & 0,19 & 0,33 & 0,62 & 1,00 & 1,40 & 1,57 & 1,60 \\
\hline $31 / 12 / 2016$ & $-0,33$ & $-0,37$ & $-0,34$ & $-0,22$ & $-0,14$ & $-0,16$ & $-0,10$ & $-0,03$ & 0,08 & 0,31 & 0,66 & 1,03 & 1,18 & 1,22 \\
\hline
\end{tabular}

Fonte: elaborazioni dell'Autore su dati Datastream.

Ai fini di un maggior approfondimento sull'applicazione del vincolo di non negatività la seguente Tabella 6 ne mostra l'applicazione alla data di riferimento del 31/12/2016. Nello specifico, la prima colonna riporta il livello vigente dei key-rates alla data di valutazione; nelle successive colonne per ogni scenario di variazione dei tassi di interesse è indicato, rispettivamente, il livello della variazione senza e con l'applicazione del vincolo di non negatività. È interessante notare che dalle fasce temporali a vista e fino a quella da oltre 3 anni a 4 anni il livello vigente dei key-rates è negativo. In tali casi in corrispondenza dell'adozione di scenari caratterizzati da variazioni negative l'applicazione del vincolo di non negatività impone di considerare una variazione pari a zero così come indicato, di recente, dall'Autorità Bancaria Europea nella FAQ del luglio 2017 (Question ID 2017_3121). Nelle fasce temporali successive, e nello specifico da quella da oltre 4 anni a 5 anni fino a quella oltre i 20 anni si registrano, invece, valori positivi ma di modesta ampiezza. In tali casi, se la variazione negativa ipotizzata è maggiore, in valore assoluto, del livello vigente alla data di valutazione l'applicazione del vincolo di non negatività richiede di considerare una variazione negativa esattamente pari ai livelli dei key-rates in modo tale da non condurre gli stessi ad assumere valori inferiori a zero. Quanto appena descritto è stato fatto, ad esempio per lo scenario parallelo dei -200 punti e per quello del $1^{\circ}$ percentile in corrispondenza di tutte le fasce temporali della matrice per scadenza e per data di riprezzamento. È interessante osservare che, dato il basso livello dei key-rates al 31/12/2016 e la conseguente applicazione del vincolo di non negatività, i due predetti scenari sono caratterizzati per ogni fascia temporale da variazioni della stessa ampiezza.

Se, invece, il livello positivo dei key-rates è maggiore, in valore assoluto, delle variazioni negative ad essi associate si utilizzano le stesse variazione negative cosi come fatto, ad esempio, per lo short rates shock down dalle fasce temporali da oltre 7 anni a 10 anni fino a quella da oltre 15 anni a 20 anni. Analoghe considerazioni valgono per lo scenario del flattener, che non è caratterizzato dall'applicazione del vincolo di non negatività in quanto le variazioni negative di cui alle fasce temporali da oltre 5 a 7 anni fino a quella oltre i 20 anni sono minori in valore assoluto del livello dei relativi key-rates. Infine, nel caso di scenari caratterizzati da variazioni positive in corrispondenza di livelli vigenti dei relativi key-rates negativi si utilizzano le stesse variazioni positive a prescindere dall'ampiezza delle stesse sulla base del principio, dettato dalle stesse indicazioni dell'Autorità Bancaria Europea, che il floor dello 0\% non si applica agli scenari di variazione in aumento. 
Tabella 6. Applicazione vincolo di non negatività al 31/12/2016

(valori percentuali)

\begin{tabular}{|c|c|c|c|c|c|c|c|c|c|c|c|c|c|c|c|c|c|}
\hline $\begin{array}{c}\text { Fasce } \\
\text { temporali }\end{array}$ & $\begin{array}{l}\text { Livello } \\
\text { key-rates }\end{array}$ & +200 & $\mathrm{VN}$ & -200 & $\mathrm{VN}$ & $99^{\circ}$ & $\mathrm{VN}$ & $1^{\circ}$ & $\mathrm{VN}$ & SRU & $\mathrm{VN}$ & SRD & $\mathrm{VN}$ & STEEP & $\mathrm{VN}$ & FLAT & $\mathrm{VN}$ \\
\hline A vista & $-0,33$ & 2,00 & 2,00 & $-2,00$ & 0,00 & 0,27 & 0,27 & $-1,09$ & 0,00 & 2,50 & 2,50 & $-2,50$ & 0,00 & $-1,63$ & 0,00 & 2,00 & 2,00 \\
\hline Fino a $1 \mathrm{~m}$ & $-0,37$ & 2,00 & 2,00 & $-2,00$ & 0,00 & 0,15 & 0,15 & $-1,27$ & 0,00 & 2,47 & 2,47 & $-2,47$ & 0,00 & $-1,60$ & 0,00 & 1,97 & 1,97 \\
\hline $1 m-3 m$ & $-0,34$ & 2,00 & 2,00 & $-2,00$ & 0,00 & 0,14 & 0,14 & $-1,28$ & 0,00 & 2,40 & 2,40 & $-2,40$ & 0,00 & $-1,52$ & 0,00 & 1,89 & 1,89 \\
\hline $3 m-6 m$ & $-0,22$ & 2,00 & 2,00 & $-2,00$ & 0,00 & 0,22 & 0,22 & $-1,37$ & 0,00 & 2,28 & 2,28 & $-2,28$ & 0,00 & $-1,40$ & 0,00 & 1,77 & 1,77 \\
\hline $6 m-1 a$ & $-0,14$ & 2,00 & 2,00 & $-2,00$ & 0,00 & 0,25 & 0,25 & $-1,42$ & 0,00 & 2,07 & 2,07 & $-2,07$ & 0,00 & $-1,19$ & 0,00 & 1,56 & 1,56 \\
\hline $1 \mathrm{a}-2 \mathrm{a}$ & $-0,16$ & 2,00 & 2,00 & $-2,00$ & 0,00 & 0,15 & 0,16 & $-1,40$ & 0,00 & 1,72 & 1,72 & $-1,72$ & 0,00 & $-0,84$ & 0,00 & 1,19 & 1,19 \\
\hline $2 a-3 a$ & $-0,10$ & 2,00 & 2,00 & $-2,00$ & 0,00 & 0,27 & 0,27 & $-1,57$ & 0,00 & 1,34 & 1,34 & $-1,34$ & 0,00 & $-0,45$ & 0,00 & 0,79 & 0,79 \\
\hline $3 a-4 a$ & $-0,03$ & 2,00 & 2,00 & $-2,00$ & 0,00 & 0,37 & 0,37 & $-1,63$ & 0,00 & 1,04 & 1,04 & $-1,04$ & 0,00 & $-0,15$ & 0,00 & 0,48 & 0,48 \\
\hline $4 a-5 a$ & 0,08 & 2,00 & 2,00 & $-2,00$ & $-0,08$ & 0,42 & 0,42 & $-1,63$ & $-0,08$ & 0,81 & 0,81 & $-0,81$ & $-0,08$ & 0,08 & 0,08 & 0,24 & 0,24 \\
\hline $5 a-7 a$ & 0,31 & 2,00 & 2,00 & $-2,00$ & $-0,31$ & 0,47 & 0,47 & $-1,58$ & $-0,31$ & 0,56 & 0,56 & $-0,56$ & $-0,31$ & 0,34 & 0,34 & $-0,02$ & $-0,02$ \\
\hline $7 a-10 a$ & 0,66 & 2,00 & 2,00 & $-2,00$ & $-0,66$ & 0,50 & 0,50 & $-1,51$ & $-0,66$ & 0,30 & 0,30 & $-0,30$ & $-0,30$ & 0,60 & 0,60 & $-0,29$ & $-0,29$ \\
\hline $10 a-15 a$ & 1,03 & 2,00 & 2,00 & $-2,00$ & $-1,03$ & 0,50 & 0,50 & $-1,59$ & $-1,03$ & 0,11 & 0,11 & $-0,11$ & $-0,11$ & 0,79 & 0,79 & $-0,49$ & $-0,49$ \\
\hline $15 a-20 a$ & 1,18 & 2,00 & 2,00 & $-2,00$ & $-1,18$ & 0,51 & 0,51 & $-1,66$ & $-1,18$ & 0,03 & 0,03 & $-0,03$ & $-0,03$ & 0,87 & 0,87 & $-0,57$ & $-0,57$ \\
\hline Oltre $20 \mathrm{a}$ & 1,22 & 2,00 & 2,00 & $-2,00$ & $-1,22$ & 0,52 & 0,52 & $-1,66$ & $-1,22$ & 0,00 & 0,00 & 0,00 & 0,00 & 0,90 & 0,90 & $-0,59$ & $-0,59$ \\
\hline
\end{tabular}

Fonte: elaborazioni dell'Autore su dati Datastream.

\section{L'esposizione dei differenti scenari di variazione dei tassi di interesse}

La seguente Tabella 7 riporta nel corso del periodo di riferimento il numero di banche esposte ai vari scenari di variazione dei tassi di interesse considerati nella presente analisi e rappresentati da quelli attualmente in vigore e quelli proposti dal Comitato di Basilea nel 2016. Come detto in precedenza, per la valuta di denominazione euro (a cui si fa riferimento nella presente analisi) vi è coincidenza tra lo scenario parallelo considerato nel quadro normativo attuale e quello proposto dal Comitato di Basilea. I vari scenari sono stati considerati in maniera congiunta a due a due in quanto caratterizzati da un certo grado di simmetria in termini di segni e variazioni sulle varie fasce temporali della matrice per scadenza e data di riprezzamento. Nello specifico, il prosieguo dell'analisi considera i singoli scenari di variazione dei tassi di interesse descritti nel corso della trattazione in base alla seguente declinazione:

a) scenario parallelo verso l'alto e verso il basso di +/- 200 punti base (parallel shock up e down);

b) $1^{\circ}$ e $99^{\circ}$ percentile delle variazioni su base annua dei tassi di interesse degli ultimi 6 anni (metodo dei percentili);

c) shock verso l'alto (basso) sul breve termine (short rates shock up e short rates shock down);

d) shock verso il basso (alto) sul breve termine e verso l'alto (basso) sul lungo termine (steepener e flattener).

In corrispondenza di ciascuna combinazione di cui ai precedenti punti da a) a d) è riportato il numero delle banche esposte e di quelle neutrali, vale a dire di quelle banche che non risultano esposte a entrambi gli scenari di variazione considerati. Sono evidenziati, inoltre, anche eventuali fenomeni di doppia esposizione, vale a dire banche esposte ad entrambi gli scenari di variazione considerati. Le ultime due colonne della Tabella 7 riportano, infine, il numero di banche esposte considerando congiuntamente, rispettivamente, le due metodologie attualmente in vigore nel quadro normativo di vigilanza prudenziale di cui ai precedenti punti a) e b) e i sei scenari proposti dal Comitato di Basilea di cui ai precedenti punti a, c) e d) 
Tabella 7. Numero di banche esposte in corrispondenza dei vari scenari di variazione dei tassi di interesse

\begin{tabular}{|ccccc|ccccc|cccc|cccccc}
\hline Anno & $\mathbf{( + 2 0 0 )}$ & $\mathbf{( - 2 0 0 )}$ & $\mathbf{N}$ & $\mathbf{D}$ & $\mathbf{9 9}^{\circ}$ & $\mathbf{1}^{\mathbf{0}}$ & $\mathbf{N}$ & $\mathbf{D}$ & SRU & SRD & $\mathbf{N}$ & $\mathbf{D}$ & STEEP & FLAT & $\mathbf{N}$ & $\mathbf{D}$ & $\mathbf{( * )}^{(* *)}$ \\
\hline 2006 & 47 & 89 & 0 & 0 & 39 & 97 & 0 & 0 & 12 & 124 & 0 & 0 & 133 & 2 & 1 & 0 & 136 & 136 \\
2007 & 29 & 107 & 0 & 0 & 21 & 113 & 2 & 0 & 5 & 131 & 0 & 0 & 133 & 3 & 1 & 1 & 136 & 136 \\
2008 & 24 & 112 & 0 & 0 & 17 & 112 & 7 & 0 & 3 & 133 & 0 & 0 & 135 & 0 & 1 & 0 & 136 & 136 \\
2009 & 33 & 99 & 4 & 0 & 21 & 115 & 1 & 1 & 8 & 127 & 2 & 1 & 136 & 0 & 0 & 0 & 136 & 136 \\
2010 & 22 & 113 & 1 & 0 & 12 & 125 & 0 & 1 & 3 & 132 & 1 & 0 & 133 & 1 & 2 & 0 & 136 & 136 \\
2011 & 39 & 83 & 14 & 0 & 31 & 103 & 2 & 0 & 8 & 129 & 0 & 1 & 135 & 0 & 1 & 0 & 136 & 136 \\
2012 & 63 & 20 & 53 & 0 & 37 & 21 & 78 & 0 & 12 & 114 & 11 & 1 & 136 & 1 & 0 & 1 & 84 & 136 \\
2013 & 75 & 25 & 36 & 0 & 43 & 31 & 62 & 0 & 12 & 121 & 3 & 0 & 136 & 2 & 0 & 2 & 106 & 136 \\
2014 & 70 & 16 & 50 & 0 & 41 & 16 & 79 & 0 & 14 & 83 & 40 & 1 & 135 & 4 & 1 & 4 & 86 & 136 \\
2015 & 67 & 6 & 63 & 0 & 34 & 6 & 96 & 0 & 8 & 61 & 68 & 1 & 136 & 3 & 0 & 3 & 73 & 136 \\
2016 & 56 & 2 & 78 & 0 & 88 & 2 & 46 & 0 & 3 & 7 & 126 & 0 & 132 & 1 & 4 & 1 & 90 & 136 \\
\hline Media & 47,7 & 61,1 & 27,2 & 0,0 & 34,9 & 67,4 & 33,9 & 0,2 & 8,0 & 105,6 & 22,8 & 0,5 & 134,5 & 1,5 & 1,0 & 1,1 & 114,1 & 136,0 \\
\hline
\end{tabular}

(*) numero banche esposte considerando congiuntamente gli scenari attualmente previsti dal quadro normativo di vigilanza prudenziale.

(**) numero banche esposte considerando congiuntamente i sei nuovi scenari proposti dal Comitato di Basilea.

Fonte: elaborazioni dell'Autore su dati di bilancio al 31/12 di ogni anno e dati Datastream.

Lo scenario parallelo dei +/- 200 punti base è caratterizzato da variazioni della stessa ampiezza e segno opposto su tutte le fasce temporali in caso di non applicazione del vincolo di non negatività. Una banca risulta, in genere, esposta allo scenario in aumento dei tassi di interesse se le riduzioni di valore economico dei fondi propri associate alle posizioni nette positive è maggiore in valore assoluto dell'incremento di valore economico associato alle posizioni nette negative. Nel caso di una banca esposta a variazioni in aumento dei tassi di interesse la riduzione di valore economico si registra, in genere, sulle fasce temporali a lungo termine dove sono presenti posizioni nette positive riconducibili alla scadenza delle quote capitali dei mutui a tasso fisso e dei titoli a tasso fisso iscritti nel portafoglio bancario che rientrano nell'orizzonte temporale in questione. $\mathrm{Nel}$ caso, invece, di banche esposte a variazioni in diminuzione dei tassi di interesse la riduzione di valore economico dei fondi propri si registra, invece, sulle fasce temporali a medio termine dove sono presenti posizioni nette negative riconducibili, principalmente, al peso dei depositi a vista e alle altre forme tecniche di raccolta a tasso fisso che scadono in tali fasce temporali.

Data la funzione di trasformazione delle scadenze, basata sul finanziamento di attività a lungo termine con passività a breve e medio termine, una banca dovrebbe risultare esposta a variazioni in aumento dei tassi di interesse. La riduzione del valore economico associata alle attività a seguito di un aumento dei tassi di interesse dovrebbe, infatti, risultare maggiore dell'incremento registrata sulle passività comportando, di conseguenza, una complessiva riduzione del valore economico dei fondi propri. Nella realtà operativa, tuttavia, data l'applicazione dei criteri di allocazione alle varie poste di bilancio, che ne modificano la durata originaria contrattuale nella prospettiva del rischio di tasso di interesse del portafoglio bancario, si rileva la presenza, nel periodo oggetto di analisi, non solo di banche esposte a variazioni in aumento dei tassi di interesse, ma anche di banche esposte a variazioni in diminuzione dei tassi di interesse. Le evidenze empiriche mostrano, inoltre, una maggiore presenza di banche esposte a variazioni in diminuzione dei tassi di interesse (61,1 in termini di valori medi sull'intero periodo) rispetto a quelle esposte a variazioni in aumento dei tassi di interesse $(47,7)$. Ciò è, principalmente, riconducibile ad una maggiore composizione del portafoglio crediti di erogazioni a tasso variabile allocate per l'intero debito residuo nella fascia temporale corrispondente alla prima data di rinegoziazione del tasso e ad un elevato ammontare di depositi a vista allocati sull'orizzonte temporale dei cinque anni.

Tuttavia, come già sottolineato dalla letteratura di riferimento [cfr. Cocozza et al. (2015), Gianfrancesco (2016) e Cerrone et al. (2017)], l'applicazione dello scenario parallelo dei $+/-200$ punti base può dare luogo al fenomeno della neutralità al rischio. Si tratta, nello specifico, di banche per le quali si rileva un incremento del valore economico dei fondi propri in corrispondenza di entrambi gli scenari di variazione in aumento e in diminuzione dei tassi di interesse. L'applicazione del vincolo di non negatività dei tassi di interesse è alla base del fenomeno della neutralità al rischio per lo scenario parallelo dei +/-200 punti base. Nel dettaglio, nell'ipotesi di utilizzo dello scenario parallelo di -200 punti base non corretto per tener conto del vincolo di non negatività la banca sarebbe stata esposta a variazioni in diminuzione dei tassi di interesse, facendo registrare una riduzione del valore economico dei fondi propri di ammontare, in valore assoluto, esattamente pari all'incremento registrato a seguito dell'applicazione dello scenario parallelo di +200 punti base. L'imposizione del vincolo di non negatività nel caso di adozione dello scenario parallelo dei -200 punti base smorza, di fatto, la riduzione di valore economico associata alle posizioni nette negative registrate nelle fasce a medio termine rendendo la banca neutrale al rischio. 
L'applicazione del vincolo di non negatività, infatti, in caso di bassi livelli dei tassi di interesse ma positivi, riduce l'ampiezza delle variazioni in diminuzione e quindi conduce, in corrispondenza di posizioni nette negative, ad una minore riduzione del valore economico dei fondi propri, oppure, nel caso di livelli sotto lo zero, impone una variazione pari a zero che non determina alcuna variazione del valore economico dei fondi propri. Le banche neutrali al rischio in assenza dell'applicazione del vincolo di non negatività sarebbero, quindi, esposte a variazioni in diminuzione dei tassi di interesse. Le evidenze empiriche riportate nella precedente Tabella 7 mostrano un numero medio di banche neutrali al rischio nel corso del periodo oggetto di analisi pari a 27,2, con un numero di casi decisamente più elevato negli ultimi due anni 2015 e 2016 pari, rispettivamente, a 63 e 78. È interessante sottolineare, a conferma di quanto detto precedentemente, che nei primi tre anni dal 2006 al 2009 non si registrano fenomeni di neutralità dato l'elevato livello dei tassi di interesse che, come detto, non ha comportato per lo scenario parallelo dei -200 punti base l'attivazione del vincolo di non negatività.

Considerazioni sostanzialmente analoghe in termini di determinanti dell'esposizione al rischio valgono per il metodo dei percentili con la differenza che gli scenari di variazione dei tassi associati al $1^{\circ}$ e al $99^{\circ}$ percentile non sono simmetrici cosi come accade invece per lo scenario parallelo dei +/-200 punti base. In tal caso l'applicazione del vincolo di non negatività non è una condizione necessaria affinché si verifichi il fenomeno della neutralità al rischio. In differenti date di valutazione, la dinamica dei tassi di interesse osservati nei 6 anni precedenti può, infatti, condurre, come effettivamente riscontrato nelle evidenze empiriche relative agli anni 2007 e 2008, a particolari scenari associati al $1^{\circ}$ e al $99^{\circ}$ percentile delle distribuzioni delle variazioni su base annua dei key-rates, che, combinati con la struttura per scadenza delle posizioni nette di una banca, generano il fenomeno della neutralità al rischio a prescindere dall'attivazione o meno del vincolo di non negatività. Anche per il metodo dei percentili, così come per lo scenario parallelo dei +/-200 punti base, si rileva un maggiore numero di banche esposte a variazioni in diminuzione dei tassi di interesse rispetto a quelle esposte a variazioni in aumento in termini di valori medi sull'intero periodo oggetto di analisi $(67,4$ vs 34,9$)$ Il numero delle banche neutrali risulta in media pari a 33,9 con valori decisamente più elevati a partire dal 2012. In corrispondenza dell'adozione di tali scenari si registra anche un caso di doppia esposizione al rischio negli anni 2009 e 2010, che conducono ad un valore medio di periodo pari a 0,2.

Gli scenari di variazione dei tassi di interessi basati sullo shock a breve termine (short rates shock up e short rates shock down) sono caratterizzati da variazioni dello stesso segno (positive e negative) dei tassi di interesse che diminuiscono via via di ampiezza a partire dalla fascia temporale a vista fino a quella oltre i 20 anni. In corrispondenza dell'ultima fascia temporale si ha una variazione pari a zero. La particolare struttura per scadenza delle posizioni nette delle banche italiane fa sì che le stesse non siano particolarmente esposte allo short rates shock up. Ciò in quanto le variazioni di maggiore ampiezza sulle fasce a medio termine dove si registrano posizioni nette negative conducono, in genere, ad un incremento di valore economico dei fondi propri maggiore, in valore assoluto, del decremento associato alle fasce temporali a lungo termine dove si registrano, invece, posizioni nette ponderate positive. Al contrario, le banche italiane sono, in genere, esposte allo short rates shock down. Ciò in quanto le variazioni di maggiore ampiezza sulle fasce a medio termine dove si registrano posizioni nette negative conducono, in genere, ad una riduzione di valore economico dei fondi propri maggiore in valore assoluto dell'incremento associato alle fasce temporali a lungo termine dove si registrano, invece, posizioni nette positive.

Le evidenze empiriche confermano quanto sopra esposto: in termini di valori medi sull'intero periodo oggetto di analisi si registra un numero di banche esposte allo short rates shock up e allo short rates shock down pari, rispettivamente, a 8,0 e 105,6. Le evidenze empiriche mostrano, inoltre, nel corso degli ultimi anni una riduzione delle banche esposte allo short rates shock down per via dell'applicazione del vincolo di non negatività, che ha smorzato, di fatto, l'effetto sopra descritto sulle fasce temporali a medio termine generando il fenomeno della neutralità al rischio. Il numero delle banche neutrali si attesta, in media sull'intero periodo, a 22,8, con un significativo incremento negli ultimi tre anni 2014, 2015 e 2016 nei quali sono registrati, rispettivamente, 40, 68 e 126 casi. Dati gli scenari in aumento e in diminuzione caratterizzati, senza l'applicazione del vincolo di non negatività, da variazioni di segno opposto ma della stessa ampiezza valgono considerazioni analoghe a quelle fatte per lo scenario parallelo dei +/-200 punti base in merito al legame tra neutralità al rischio e applicazione del vincolo di non negatività. Inoltre, si rilevano nel corso del periodo alcuni casi di doppia esposizione, che conducono ad un valore medio di 0,5 .

Lo steepener e il flattener sono scenari di variazione dei tassi di interesse caratterizzati da differente ampiezza e segno sulle varie fasce temporali della matrice per scadenza e per data di riprezzamento. Nello specifico, lo steepener è caratterizzato da variazioni negative via via decrescenti in ampiezza dalla fascia temporale a vista fino a quella da oltre 3 anni a 4 anni e da variazioni positive via via crescenti in ampiezza dalla fascia da oltre 4 anni a 5 anni fino a quella oltre i 20 anni. Il flattener è, invece, caratterizzato da variazioni positive via via decrescenti in ampiezza dalla fascia temporale a vista fino a quella da oltre 4 anni a 5 anni e da variazioni negative via via crescenti in ampiezza dalla fascia da oltre 5 anni a 7 anni a oltre i 20 anni. La particolare struttura per scadenza delle posizioni nette delle banche italiane fa sì che le stesse non siano particolarmente esposte al flattener. Ciò in quanto le variazioni positive sulle fasce a medio termine e negative su quelle a lungo termine conducono ad un incremento del valore economico dei fondi propri date le relative posizioni nette, rispettivamente, negative e positive ad esse associate. Di conseguenza l'applicazione di tale scenario conduce, in genere, su 
tutte le fasce temporali a medio e lungo termine della matrice per scadenza e per data di riprezzamento ad un incremento del valore economico dei fondi propri. Al contrario, le banche italiane, invece, risultano, in genere, esposte allo steepener in quanto la particolare struttura per scadenza delle posizioni nette fa sì che si registri una riduzione di valore economico sia sulle fasce a medio termine, data l'applicazione di variazioni negative a posizioni nette negative (ad eccezione della fascia temporale da 4 a 5 anni), sia su quelle a lungo termine, data l'applicazione di variazioni positive a posizione nette positive.

Quanto detto trova conferma nelle evidenze empiriche del periodo oggetto di analisi: in termini di valori medi si registra un numero di banche esposte allo steepener ed al flattener pari, rispettivamente, a 134,5 e 1,5. Non si registrano, inoltre, elevati fenomeni di neutralità al rischio: in termini di valori medi sull'intero periodo le banche neutrali si attestano a 1,0. L'applicazione del vincolo di non negatività, così come per il metodo dei percentili, non è una condizione necessaria affinché si verifichi il fenomeno della neutralità. Al riguardo è interessante osservare che il flattener è caratterizzato da variazioni negative sulle fasce a lungo termine più basse in valore assoluto dei relativi livelli dei key-rates osservati nel corso degli anni. Tale scenario non ha, quindi, richiesto l'applicazione del vincolo di non negatività. Lo steepener è caratterizzato, invece, da variazioni negative sulle fasce a breve e medio termine e positive su quelle a lungo termine. L'applicazione del vincolo di non negatività in contesti di bassi livelli dei tassi di interesse riduce (nel caso di livelli dei tassi vigenti alla data di valutazione positivi) o annulla (nel caso di livelli dei tassi vigenti alla data di valutazione negativi) la corrispondente riduzione di valore economico che si avrebbe in corrispondenza delle fasce temporali a medio termine attenuando, di fatto, la complessiva esposizione al rischio determinata sulle fasce a lungo termine dove si registra una riduzione di valore economico a seguito dell'applicazione di variazioni positive a posizioni nette positive. Inoltre, si rilevano nel corso del periodo alcuni casi di doppia esposizione per un valore medio di 1,1 .

Infine, è interessante osservare le evidenze delle ultime due colonne delle Tabella 7 che riportano il numero di banche esposte considerando congiuntamente gli scenari imposti dall'attuale quadro normativo di vigilanza prudenziale e i sei nuovi scenari proposti dal Comitato di Basilea. In relazione alla vigente architettura regolamentare si segnala a partire dal 2012 la presenza di banche neutrali al rischio in corrispondenza dell'applicazione sia dello scenario parallelo dei $+/-200$ punti base che del metodo dei percentili con un conseguente capitale interno da porre a presidio di tale tipologia di rischio in sede ICAAP pari a zero. L'applicazione, invece, degli scenari proposti dal Comitato di Basilea fa sì che l'intero campione sia sempre esposto al rischio in questione con un conseguente ammontare di capitale interno maggiore di zero. L'approccio proposto dal Comitato di Basilea è, quindi, da ritenere più prudenziale di quello attualmente previsto dal quadro normativo di vigilanza prudenziale.

\section{Le evidenze in termini di livello dell'indicatore di rischio regolamentare}

La seguente Tabelle 8 riporta il livello medio dell'indicatore di rischio calcolato sulle sole banche esposte in corrispondenza dei singoli scenari di variazione dei tassi di interesse.

Tabella 8. Livello medio dell'indicatore di rischio delle banche esposte ai vari scenari di variazione dei tassi di interesse (valori percentuali)

\begin{tabular}{ccc|cc|cc|ccc|c}
\hline Anno & $\mathbf{( + 2 0 0 )}$ & $\mathbf{( - 2 0 0 )}$ & $\mathbf{9 9}^{\circ}$ & $\mathbf{1}^{\mathbf{0}}$ & SRU & SRD & STEEP & FLAT & $\mathbf{( * )}^{*} \mathbf{( * *}^{*}$ \\
\hline 2006 & 14,34 & 9,22 & 6,58 & 9,31 & 2,13 & 5,46 & 4,45 & 0,50 & 11,56 & 11,37 \\
2007 & 10,90 & 8,23 & 4,82 & 8,74 & 2,31 & 5,71 & 3,78 & 2,08 & 9,67 & 9,47 \\
2008 & 11,74 & 7,78 & 4,46 & 5,20 & 2,12 & 6,40 & 4,35 & 0,00 & 8,49 & 9,40 \\
2009 & 14,02 & 7,94 & 7,79 & 10,52 & 1,44 & 6,46 & 5,04 & 0,00 & 12,14 & 10,44 \\
2010 & 8,34 & 8,55 & 4,07 & 10,61 & 1,15 & 7,09 & 4,60 & 10,33 & 11,10 & 9,78 \\
2011 & 13,83 & 5,94 & 5,54 & 6,76 & 2,00 & 6,64 & 5,90 & 0,00 & 9,07 & 10,14 \\
2012 & 8,58 & 2,61 & 2,10 & 2,79 & 1,40 & 2,78 & 5,01 & 0,47 & 7,13 & 6,93 \\
2013 & 9,18 & 3,87 & 2,61 & 3,83 & 2,71 & 3,96 & 5,80 & 1,13 & 7,62 & 8,49 \\
2014 & 9,95 & 1,28 & 2,56 & 1,28 & 2,18 & 1,23 & 5,32 & 1,40 & 8,34 & 7,42 \\
2015 & 7,61 & 0,42 & 1,96 & 0,42 & 2,85 & 0,83 & 5,13 & 2,12 & 7,02 & 6,52 \\
2016 & 9,29 & 2,28 & 2,41 & 2,28 & 2,01 & 0,24 & 5,34 & 0,11 & 6,17 & 6,54 \\
\hline media & 10,71 & 5,28 & 4,08 & 5,61 & 2,03 & 4,25 & 4,98 & 1,65 & 8,94 & 8,77 \\
\hline
\end{tabular}

$(*)$ massimo livello dell'indicatore di rischio per le banche esposte considerando congiuntamente gli scenari attualmente previsti dal quadro normativo di vigilanza prudenziale.

$(* *)$ massimo livello dell'indicatore di rischio per le banche esposte considerando congiuntamente i sei nuovi scenari proposti dal Comitato di Basilea.

Fonte: elaborazioni dell'Autore su dati di bilancio al 31/12 di ogni anno e dati datastream.

Le evidenze empiriche sull'intero periodo oggetto di analisi mostrano, in termini di valori medi, che per le banche esposte a variazioni in aumento dei tassi di interesse lo scenario parallelo dei +200 punti base conduce ad un livello medio 
dell'indicatore di rischio più elevato pari al 10,71\% rispetto al $99^{\circ}$ percentile e allo short rates shock up, che presentano, invece, valori medi più bassi pari, rispettivamente, a 4,08\% e a 2,03\%. Il dato relativo allo short rates shock up risente, tuttavia, della bassa propensione delle banche commerciali italiane ad essere esposte a tale tipologia di scenario per via della particolare struttura per scadenza delle posizioni nette come evidenziato nel precedente paragrafo. È interessante osservare che lo scenario parallelo dei +200 punti base presenta un valore medio dell'indicatore di rischio più elevato dello scenario corrispondente al $99^{\circ}$ percentile e allo short rates shock up in tutti i singoli anni oggetto di analisi. L'ampiezza delle variazioni associate ai vari scenari considerati è alla base di tali evidenze. Nello specifico, lo scenario parallelo dei +200 punti base presenta, per tutte le fasce temporali della matrice per scadenza e data di riprezzamento, variazioni dei key-rates di maggiore ampiezza soprattutto sulle fasce temporali a lungo termine. In corrispondenza di tale scenario, infatti, la riduzione del valore economico associato alle posizioni nette positive sulle fasce temporali lungo termine impatta sull'esposizione al rischio in maniera più accentuata dell'incremento di valore economico registrato in corrispondenza delle posizioni nette negative sulle fasce temporali a medio termine rispetto a quanto avviene per lo scenario del $99^{\circ}$ percentile e dello short rates shock up.

In relazione, invece, alle banche esposte a variazioni in diminuzione dei tassi di interesse lo scenario del $1^{\circ}$ percentile è quello che conduce ad un livello medio dell'indicatore più alto pari al 5,61\%. I valori sono di poco superiori a quelli ottenuti a seguito dell'applicazione dello scenario parallelo dei -200 punti base e dello short rates shock down pari, rispettivamente, al $5,28 \%$ e al 4,25\%. È interessante osservare che le differenze tra i tre scenari considerati sono meno accentuate rispetto a quanto osservato per le banche esposte a variazioni in aumento. In tale contesto, inoltre, l'attivazione del vincolo di non negatività ha condotto, a partire dal 2009, ad un progressivo allineamento dell'incremento di valore economico dei fondi propri sulle fasce a breve e medio termine. Di conseguenza le differenze in termini di esposizione al rischio sono state, in larga parte, riconducibili all'impatto dei singoli scenari sulle fasce temporali a lungo termine dove si registra, in genere, un incremento di valore economico data la presenza di posizione nette positive. Inoltre, dal 2014 al 2016 si sono registrate variazioni analoghe su tutte le fasce temporali della matrice per scadenza e per data di riprezzamento in corrispondenza dello scenario dei -200 punti base e del $1^{\circ}$ percentile, che hanno condotto, di conseguenza, ad uno stesso livello medio dell'indicatore di rischio.

Inoltre, per quel che concerne lo steepener si registra un valore medio sull'intero periodo oggetto di analisi dell'indicatore di rischio pari al 4,98\%. L'ampiezza contenuta delle variazioni unitamente alla progressiva applicazione del vincolo di non negatività, che ha smorzato, di fatto, l'impatto delle variazioni negative sulle fasce temporali a breve e medio termine è alla base delle evidenze ottenute. Si rileva al riguardo una elevata differenza rispetto al valore medio ottenuto in corrispondenza dello scenario parallelo dei +200 punti base pari al 10,71\% mentre le distanze rispetto ai valori medi ottenuti in corrispondenza degli altri scenari sono più contenute. Il dato ottenuto per lo steepener è rappresentativo della quasi totalità del campione data l'elevata propensione delle banche commerciali italiane ad essere esposte a tale scenario. Lo steepener, infatti, come detto nel corso della trattazione, conduce sia sulle fasce a medio termine, con eccezione di quella da oltre 4 anni a 5 anni, sia su quelle a lungo termine un incremento di valore economico dei fondi propri data la particolare struttura per scadenza delle posizioni nette delle banche commerciali italiane. Le evidenze relative, invece, all'applicazione del flattener mostrano un valore medio dell'indicatore di rischio abbastanza contenuto che si attesta a 1,65\%. Il dato risente, tuttavia, della bassa propensione delle banche commerciali italiane ad essere esposte a tale tipologia di scenario per via della particolare struttura per scadenza delle posizioni nette. Infine, è interessante osservare i dati relativi alle ultime due colonne della Tabella 8, che riportano il massimo livello dell'indicatore di rischio per le banche esposte considerando congiuntamente, rispettivamente, le due metodologie attualmente in vigore e i sei nuovi scenari proposti dal Comitato di Basilea. Il livello medio complessivo risulta di poco maggiore per la combinazione degli scenari attualmente previsti dal quadro normativo di vigilanza prudenziale rappresentati, come noto, dallo scenario parallelo dei +/-200 punti base e dal metodo dei percentili. Il dato, tuttavia, non deve essere interpretato come un elemento di maggiore prudenzialità dato che, come evidenziato nella precedente Tabella 7, in situazioni di basso livello di tassi di interesse si verificano elevati casi di doppia neutralità,vale a dire di banche non esposte in corrispondenza di entrambe le due metodologie attualmente in vigore. I valori più elevati in termini di valori medi dell'indicatore di rischio associati alla combinazione degli scenari attualmente in vigore sono riconducibili alle banche esposte a variazioni in diminuzione dei tassi di interesse che presentano un valore dell'indicatore di rischio più alto a seguito dell'applicazione del metodo dei percentili che è considerato nell'attuale quadro normativo di vigilanza prudenziale e non nell'ambito della nuova proposta formulata dal Comitato di Basilea.

\section{Un'applicazione su una singola banca al 31/12/2016}

Un'applicazione ai dati di una singola banca può aiutare a comprendere i concetti espressi nel corso della trattazione. La seguente Tabella 9 riporta il calcolo dell'indicatore di rischio in corrispondenza dello scenario parallelo dei $+/-200$ punti base e del metodo dei percentili; la successiva Tabella 10 in corrispondenza, invece, dello shock sul breve termine (short rates shock up e down), dello steepener e del flattener. Dalla seconda alla quarta colonna di ciascuna tabella sono riportati il livello dei tassi di interesse vigente alla data di valutazione, le posizioni nette e le duration associate alle varie fasce temporali della 
matrice per scadenza e per data di riprezzamento. Successivamente, per ogni singolo scenario sono considerate le relative variazioni dei tassi di interesse dopo l'applicazione del vincolo di non negatività e le posizioni nette ponderate. Queste ultime sono ottenute moltiplicando le posizioni nette (terza colonna), per i coefficienti di duration (quarta colonna) e le variazioni dei tassi di interesse, corrette per il vincolo di non negatività, associate ad ogni singolo scenario. Le ultime due righe di ogni tabella riportano in corrispondenza di ogni scenario la somma delle posizioni nette ponderate e il livello dell'indicatore di rischio ottenuto rapporto la predetta somma al livello dei fondi propri alla data di valutazione.

Tabella 9. Calcolo dell'indicatore di rischio singola banca al 31/12/2016 mediante l'applicazione dello scenario parallelo dei $+/-200$ punti base e del metodo dei percentili

(PN, $\triangle \mathrm{PN}$ e $\triangle \mathrm{VE}$ in migliaia di euro - key-rates $\mathrm{e}$ IR in valori percentuali)

\begin{tabular}{|c|c|c|c|c|c|c|c|c|c|c|c|}
\hline $\begin{array}{c}\text { fasce } \\
\text { temporali }\end{array}$ & $\begin{array}{l}\text { livello } \\
\text { key-rate }\end{array}$ & $\mathrm{PN}$ & Duration & +200 & $\Delta \mathrm{PN}$ & -200 & $\Delta \mathrm{PN}$ & $99^{\circ}$ & $\Delta \mathrm{PN}$ & $1^{\circ}$ & $\Delta \mathrm{PN}$ \\
\hline A vista & $-0,33$ & 28.786 & 0,00 & 2,00 & 0 & 0,00 & 0 & 0,27 & 0 & 0,00 & 0 \\
\hline fino a $1 \mathrm{~m}$ & $-0,37$ & 166.202 & 0,04 & 2,00 & 132 & 0,00 & 0 & 0,15 & 10 & 0,00 & 0 \\
\hline $1 m-3 m$ & $-0,34$ & 332.405 & 0,16 & 2,00 & 1.055 & 0,00 & 0 & 0,14 & 74 & 0,00 & 0 \\
\hline $3 m-6 m$ & $-0,22$ & -41.397 & 0,36 & 2,00 & -298 & 0,00 & 0 & 0,22 & -33 & 0,00 & 0 \\
\hline $6 m-1 a$ & $-0,14$ & -23.494 & 0,71 & 2,00 & -334 & 0,00 & 0 & 0,25 & -42 & 0,00 & 0 \\
\hline $1 \mathrm{a}-2 \mathrm{a}$ & $-0,16$ & -121.305 & 1,38 & 2,00 & -3.348 & 0,00 & 0 & 0,16 & -274 & 0,00 & 0 \\
\hline $2 a-3 a$ & $-0,10$ & -121.305 & 2,25 & 2,00 & -5.459 & 0,00 & 0 & 0,27 & -731 & 0,00 & 0 \\
\hline $3 a-4 a$ & $-0,03$ & -121.305 & 3,07 & 2,00 & -7.448 & 0,00 & 0 & 0,37 & -1.384 & 0,00 & 0 \\
\hline $4 a-5 a$ & 0,08 & -121.305 & 3,85 & 2,00 & -9.340 & $-0,08$ & 350 & 0,42 & -1.957 & $-0,08$ & 350 \\
\hline $5 a-7 a$ & 0,31 & 29.760 & 5,08 & 2,00 & 3.024 & $-0,31$ & -475 & 0,47 & 710 & $-0,31$ & -475 \\
\hline $7 a-10 a$ & 0,66 & 44.641 & 6,63 & 2,00 & 5.919 & $-0,66$ & -1.962 & 0,50 & 1.477 & $-0,66$ & -1.962 \\
\hline $10 a-15 a$ & 1,03 & 4.548 & 8,92 & 2,00 & 811 & $-1,03$ & -418 & 0,50 & 202 & $-1,03$ & -418 \\
\hline $15 a-20 a$ & 1,18 & 4.548 & 11,21 & 2,00 & 1.020 & $-1,18$ & -600 & 0,51 & 262 & $-1,18$ & -600 \\
\hline Oltre $20 \mathrm{a}$ & 1,22 & 4.548 & 13,01 & 2,00 & 1.183 & $-1,22$ & -721 & 0,52 & 307 & $-1,22$ & -721 \\
\hline \multicolumn{2}{|c|}{$\Delta \mathrm{VE}$} & & & & -13.082 & & -3.825 & & -1.378 & & -3.825 \\
\hline \multicolumn{2}{|c|}{$\mathrm{IR}=\Delta \mathrm{VE} / \mathrm{FP}$} & & & & $-5,87$ & & $-1,72$ & & $-0,62$ & & $-1,72$ \\
\hline
\end{tabular}

Fonte: elaborazioni dell'Autore su dati di bilancio al 31/12/2016 e dati Datastream.

Tabella 10. Calcolo dell'indicatore di rischio singola banca al 31/12/2016 mediante l'applicazione degli scenari short rates shock up e down, steeepener e flattener

(PN, $\mathrm{PN}$ e $\Delta \mathrm{VE}$ in migliaia di euro - key-rates $\mathrm{e}$ IR in valori percentuali)

\begin{tabular}{|c|c|c|c|c|c|c|c|c|c|c|c|}
\hline fasce temporali & $\begin{array}{c}\text { lvello } \\
\text { key-rates }\end{array}$ & PN & Duration & SRU & $\Delta \mathrm{PN}$ & SRD & $\Delta \mathrm{PN}$ & STEEP & $\Delta \mathrm{PN}$ & FLATT & $\Delta \mathrm{PN}$ \\
\hline A vista & $-0,33$ & 28.786 & 0,00 & 2,50 & 0 & 0,00 & 0 & 0,00 & 0 & 2,00 & 0 \\
\hline fino a $1 \mathrm{~m}$ & $-0,37$ & 166.202 & 0,04 & 2,47 & 163 & 0,00 & 0 & 0,00 & 0 & 1,97 & 130 \\
\hline $1 \mathrm{~m}-3 \mathrm{~m}$ & $-0,34$ & 332.405 & 0,16 & 2,40 & 1.265 & 0,00 & 0 & 0,00 & 0 & 1,89 & 999 \\
\hline $3 m-6 m$ & $-0,22$ & -41.397 & 0,36 & 2,28 & -339 & 0,00 & 0 & 0,00 & 0 & 1,77 & -263 \\
\hline $6 \mathrm{~m}-1 \mathrm{a}$ & $-0,14$ & -23.494 & 0,71 & 2,07 & -346 & 0,00 & 0 & 0,00 & 0 & 1,56 & -259 \\
\hline $1 a-2 a$ & $-0,16$ & -121.305 & 1,38 & 1,72 & -2.876 & 0,00 & 0 & 0,00 & 0 & 1,19 & -1.987 \\
\hline $2 a-3 a$ & $-0,10$ & -121.305 & 2,25 & 1,34 & -3.652 & 0,00 & 0 & 0,00 & 0 & 0,79 & -2.161 \\
\hline $3 a-4 a$ & $-0,03$ & -121.305 & 3,07 & 1,04 & -3.881 & 0,00 & 0 & 0,00 & 0 & 0,48 & -1.802 \\
\hline $4 a-5 a$ & 0,08 & -121.305 & 3,85 & 0,81 & -3.791 & $-0,08$ & 350 & 0,08 & -375 & 0,24 & -1.140 \\
\hline $5 a-7 a$ & 0,31 & 29.760 & 5,08 & 0,56 & 843 & $-0,31$ & -475 & 0,34 & 509 & $-0,02$ & -30 \\
\hline $7 a-10 a$ & 0,66 & 44.641 & 6,63 & 0,30 & 884 & $-0,30$ & -884 & 0,60 & 1.771 & $-0,29$ & -857 \\
\hline $10 a-15 a$ & 1,03 & 4.548 & 8,92 & 0,11 & 45 & $-0,11$ & -45 & 0,79 & 320 & $-0,49$ & -197 \\
\hline $15 a-20 a$ & 1,18 & 4.548 & 11,21 & 0,03 & 16 & $-0,03$ & -16 & 0,87 & 443 & $-0,57$ & -289 \\
\hline Oltre 20a & 1,22 & 4.548 & 13,01 & 0,00 & 3 & 0,00 & -3 & 0,90 & 530 & $-0,59$ & -352 \\
\hline \multicolumn{2}{|c|}{$\Delta \mathrm{VE}$} & & & & -11.666 & & -1.072 & & 3.198 & & -8.208 \\
\hline \multicolumn{2}{|c|}{$\mathrm{IR}=\Delta \mathrm{VE} / \mathrm{FP}$} & & & & $-5,23$ & & $-0,48$ & & 1,43 & & $-3,68$ \\
\hline
\end{tabular}

Fonte: elaborazioni dell'Autore su dati di bilancio al 31/12/2016 e dati Datastream. 
La banca oggetto di analisi risulta neutrale al rischio in corrispondenza dello scenario parallelo dei +/-200 punti base, del metodo dei percentili e dello shock sul breve termine (short rates shock up e short rates shock down). Si tratta, quindi, di una banca esposta, in generale, per la particolare struttura per scadenza delle posizioni nette a variazioni in diminuzione dei tassi di interesse. In corrispondenza dello scenario parallelo dei -200 punti base e dello short rates shock down in assenza dell'applicazione del vincolo di non negatività si avrebbe, infatti, una riduzione del valore economico esattamente pari all'incremento registrato per lo scenario parallelo dei +200 punti base e dello shock sul breve termine verso l'alto. La banca sarebbe, inoltre, esposta allo scenario del $1^{\circ}$ percentile non corretto per il vincolo di non negatività. In tal caso, tuttavia, come già detto nel corso della trattazione non si ha una esposizione simmetrica rispetto al $99^{\circ}$ percentile. In corrispondenza di tutti e tre gli scenari, l'applicazione del vincolo di non negatività a partire dalla fascia temporale da 1 mese a 3 mesi fino a quella da 4 anni a 5 anni dove si registrano posizione nette negative annulla (fino alla fascia temporale da 3 a 4 anni) e smorza (nella fascia temporale da 4 a 5 anni) la riduzione di valore economico che si avrebbe in caso di utilizzo delle variazioni non corrette. È interessante osservare che per tutti e tre gli scenari di variazione in diminuzione il livello negativo dei tassi di interesse vigente alla data di valutazione fino alla fascia temporale da 3 anni a 4 anni impone l'applicazione di una variazione pari a zero. In aggiunta, il basso livello dei tassi di interesse nelle fasce temporali successive fa sì che lo scenario corrispondente ai 200 punti base e quello del $1^{\circ}$ percentile sono caratterizzati su tutte le fasce temporali della matrice per scadenza e per data di riprezzamento da variazioni analoghe.

Infine, la banca oggetto di analisi risulta esposta allo steepener data la riduzione di valore economico registrata sulle fasce a lungo termine da 5 a 7 anni fino a quella oltre i 20 anni. Nelle fasce temporali a vista fino a quella da 3 a 4 anni si registra una variazione di valore economico pari a zero data l'applicazione del vincolo di non negatività su un livello vigente dei tassi alla data di valutazione negativo e un moderato incremento di valore economico nella fascia temporale da 4 a 5 anni data l'applicazione di una variazione positiva di 8 punti base. È interessante osservare che in tal caso l'applicazione del vincolo di non negatività smorza l'ulteriore riduzione di valore economico che si sarebbe registrata sulle fasce temporali da 3 mesi a 6 mesi fino a quella da 3 anni a 4 anni dove si rilevano posizioni nette negative. La complessiva esposizione al rischio sarebbe stata, quindi, maggiore in caso di non applicazione del vincolo di non negatività. Infine, la banca non risulta esposta al flatteneer in quanto l'applicazione di tale scenario conduce sia sulle fasce a breve e medio termine (con eccezione delle prime tre fasce temporali) che su quelle a lungo termine ad incrementi del valore economico dei fondi propri dato il segno delle variazioni dei key-rates (positive sul breve e medio termine e negative sul lungo termine) e delle posizioni nette (negative a partire dalla fascia temporale da 3 a 6 mesi fino a quella da 4 a 5 anni e positive sul lungo termine oltre i 5 anni). Le variazioni negative dei tassi di interesse sulle fasce a lungo termine in quanto di minore ampiezza rispetto al livello dei tassi vigente alla data di valutazione non sono impattate dall'applicazione del vincolo di non negatività.

\section{Conclusioni ed implicazioni di policy}

La corretta comprensione delle determinanti dell'esposizione al rischio di tasso di interesse del portafoglio bancario è di fondamentale importanza ai fini della definizione delle appropriate strategie di ALM funzionali al raggiungimento degli obiettivi strategici della banca. Le proposte di modifica al quadro normativo di vigilanza prudenziale richiedono alle banche, quindi, di analizzare le relative implicazioni sulla complessiva esposizione al rischio e di calibrare, sulla base del nuovo dettato regolamentare, gli obiettivi di rischio e le relative soglie di attenzione in sede di RAF e di sistema di allocazione del capitale sia sull'intera banca che sulle differenti linee di business. Le scelte in termini di data di scadenza e di revisione del tasso di interesse degli impieghi erogati e delle forme tecniche di raccolta emesse nonché dei titoli detenuti nel portafoglio di proprietà e, quindi, in generale delle strategie di $A L M$ poste in essere dalla banca dovrebbe fin da ora essere effettuata anche sulla base dei nuovi scenari di variazione dei tassi di interesse introdotti nelle recenti proposte di modifica da parte del Comitato di Basilea al fine di verificarne la sostenibilità, tenendo conto della eventuale riduzione della soglia di allerta dal $20 \%$ al $15 \%$

Le evidenze empiriche nel periodo 2006-2016 confermano, come già sottolineato dalla precedente letteratura di riferimento, la significativa presenza, in un contesto di bassi livelli dei tassi di interesse, del fenomeno della neutralità al rischio per le metodologie attualmente previste dal quadro normativo di vigilanza prudenziale e rappresentate dallo scenario parallelo dei +/- 200 punti base e dal metodo dei percentili. A partire dal 2012 si registrano, inoltre, casi di doppia neutralità rappresentati da banche non esposte al rischio in corrispondenza di entrambe le due metodologie regolamentari. L'introduzione di quattro nuovi scenari rappresentanti dallo shock a breve termine verso l'altro e verso il basso (short rates shock up e short rates shock down) e dello shock verso il basso sul breve termine e verso l'alto sul lungo termine (steepener) e verso l'alto sul breve termine e il basso sul lungo termine (flattener), in aggiunta a quello dello spostamento parallelo dei +/200 punti base, conferisce un maggior grado di prudenzialità all'architettura regolamentare di vigilanza. Le 136 banche del campione risultano, infatti, sempre esposte ad almeno uno di questi scenari. 
Ciò, è in larga parte, riconducibile allo steepener che è caratterizzato da variazioni negative via via decrescenti in termini di ampiezza dalla fascia temporale a vista fino a quella da 3 a 4 anni e variazioni positive via via crescenti, invece, sulle fasce da 4 a 5 anni fino ad oltre i 20 anni. Le banche italiane risultano, nella maggior parte dei casi, esposte a tale scenario data la particolare struttura per scadenza delle posizioni nette che conduce ad un incremento di valore economico dei fondi propri sul medio termine (con l'eccezione della fascia temporale da 4 a 5 anni) a seguito dell'applicazione di variazioni negative a posizioni nette negative ed ad una riduzione di valore economico dei fondi propri sul lungo termine a seguito dell'applicazione di variazioni positive a posizioni nette positive. In termini di valori medi sull'intero periodo oggetto di analisi si registra un numero di banche esposte allo scenario dello steepener pari rispettivamente a 134,5 che rappresenta la quasi totalità del campione. Il basso livello dei tassi di interesse registrato negli ultimi anni ha, tuttavia, condotto, mediante l'applicazione del vincolo di non negatività, ad annullare (nel caso di un livello dei tassi di interesse sotto lo zero) o ad attenuare (nel caso di bassi livelli ma comunque positivi) l'impatto di tale scenario sulle fasce temporali a breve e medio termine conducendo, quindi, ad una complessiva riduzione dell'esposizione al rischio.

Viceversa le banche italiane non risultano, in genere, esposte al flattener caratterizzato, invece, da variazioni positive via via decrescenti in termini di ampiezza dalla fascia temporale a vista fino a quella da 4 a 5 anni e da variazioni negative via via crescenti a partire, invece, dalla fascia temporale da 5 a 7 anni fino a quella oltre i 20 anni. La particolare struttura per scadenza delle posizioni nette di una tipica banca commerciale italiana fa sì che si registri, infatti, un incremento di valore economico sia sulle fasce a medio termine, data l'applicazione di variazioni positive a posizioni nette negative, sia su quelle a lungo termine, data l'applicazione di variazioni negative a posizioni nette positive, che rendono, quindi, la banca non esposta a tale scenario. In termini di valori medi sull'intero periodo oggetto di analisi si registra, infatti, un numero di banche esposte pari a 1,5. Lo scenario in questione non risente, inoltre, dell'applicazione del vincolo di non negatività. Le variazioni negative sulle fasce a lungo termine sono, infatti, risultate in tutto il periodo oggetto di analisi maggiori, in valore assoluto, del livello dei tassi di interesse vigente alle varie date di valutazione.

Gli altri due nuovi scenari introdotti dal Comitato di Basilea rappresentati dallo shock sul breve termine verso l'alto (short rates shock up) e verso il basso (short rates shock down) sono caratterizzati da variazioni dei tassi di interesse dello stesso segno, rispettivamente positive e negative, che diminuiscono di ampiezza via via che si passa dalle fasce temporali a breve termine fino a quelle a lungo termine. Le banche italiane, data la particolare struttura per scadenza delle posizioni nette, risultano particolarmente esposte allo short rates shock down poiché le variazioni negative di maggiore ampiezza in valore assoluto sulle fasce a medio termine, dove si registrano posizioni nette negative, conducono, in genere, ad una riduzione del valore economico dei fondi propri maggiore, in valore assoluto, dell'incremento registrato sulle fasce a lungo termine dove si rilevano, invece, posizioni nette positive. L'applicazione del vincolo di non negatività negli ultimi anni caratterizzati da bassi livelli dei tassi di interesse ha generato fenomeni di neutralità al rischio riducendo, quindi, il numero di banche esposte a tale scenario. In termini di valori medi sull'intero periodo oggetto di analisi le banche esposte allo short rates shock down si sono attestate a 105,6. Le banche italiane non risultano, invece, particolarmente esposte, data la struttura per scadenza delle posizioni nette, allo short rates shock up in quanto le variazioni positive di maggiore ampiezza sulle fasce a medio termine, dove si registrano posizioni nette negative, conducono, in genere, ad un incremento del valore economico dei fondi propri maggiore in valore assoluto della riduzione registrata sulle fasce temporali a lungo termine dove si rilevano, invece, posizioni nette positive. In termini di valori medi, infatti, il numero di banche esposte a tale scenario nell'intero periodo oggetto di analisi si attesta a 8,0 .

In termini di livelli dell'indicatore di rischio per le banche esposte a variazioni in aumento dei tassi di interesse lo scenario parallelo dei +200 punti base conduce ad un livello medio dell'indicatore di rischio più elevato pari al $10,71 \%$ rispetto al $99^{\circ}$ percentile e allo short rates shock up, che presentano valori medi più bassi pari, rispettivamente, a $4,08 \%$ e a 2,03\%. L'ampiezza delle variazioni associate ai vari scenari considerati è alla base di tali evidenze. In relazione, invece, alle banche esposte a variazioni in diminuzione dei tassi di interesse lo scenario del $1^{\circ}$ percentile è quello che conduce ad un livello medio dell'indicatore più alto pari al 5,61\%. I valori sono di poco superiori a quelli ottenuti a seguito dell'applicazione dello scenario parallelo dei -200 punti base e dello short rates shock down pari, rispettivamente, al 5,28\% e al 4,25\%. È interessante osservare che le differenze tra i tre scenari considerati sono meno accentuate rispetto a quanto osservato per le banche esposte a variazioni in aumento. Infine, per quel che concerne lo steepener si registra un valore medio sull'intero periodo oggetto di analisi dell'indicatore di rischio pari al 4,94\%. L'ampiezza contenuta delle variazioni unitamente alla progressiva applicazione del vincolo di non negatività, che ha smorzato, di fatto, l'impatto delle variazioni negative sulle fasce temporali a medio termine è alla base delle evidenze ottenute. Si rileva per tale scenario una elevata differenza rispetto al valore medio ottenuto in corrispondenza dello scenario parallelo dei +200 punti base pari al 10,71\% mentre le distanze rispetto ai valori medi ottenuti in corrispondenza degli altri scenari sono, invece, più contenute.

Le tematiche oggetto di discussione sono di particolare rilevanza per gli operatori del settore che, a seguito delle innovazioni regolamentari, devono non solo misurare l'esposizione al rischio della banca sulla base dei nuovi scenari di variazione dei tassi di interesse proposti dal Comitato di Basilea, ma anche comprenderne, allo stesso tempo, le relative 
determinanti e le conseguenti implicazioni gestionali e di business. Il presupposto logico per la definizione di appropriate scelte di ALM funzionali al raggiungimento degli obiettivi strategici declinati nel piano industriale, dato il capitale libero disponibile, è rappresentato da una adeguata diffusione della cultura del rischio tra le varie funzioni aziendali interessate. In tale contesto, una maggiore consapevolezza degli impatti in termini di capitale interno da porre a presidio del rischio in questione derivante dagli impieghi erogati, dei titoli iscritti nel portafoglio di proprietà e dalle differenti forme tecniche di raccolta emesse contribuisce a rendere le differenti strutture operative maggiormente consapevoli della relazione di interdipendenza tra l'attivo e il passivo bancario e, allo stesso tempo, ad avere una visione più integrata e trasversale della banca, facendo leva su una maggiore e più efficiente interazione tra $\mathrm{i}$ vari processi aziendali. Le tematiche oggetto di analisi rappresentano, infine, uno stimolo per la comunità accademica e, nello specifico, per gli studiosi di risk management collocati sulla linea di demarcazione tra ricerca teorica ed applicata ed incentivati a spostare più in avanti la frontiera dell'indagine scientifica nella prospettiva di consentire una sempre e più consapevole assunzione del rischio da parte degli intermediari.

Igor Gianfrancesco

\section{Bibliografia}

- Abdymomunov A., Gerlach J., 2014. Stress testing interest rate risk exposure, in Journal of Banking and Finance 49, $287-301$.

- AIFIRM (2016), Risposta al documento di consultazione Interest rate risk in the banking book del Comitato di Basilea sulla vigilanza bancaria, in Newsletter AIFIRM, Anno 10, Numero 3. Il position paper è scaricabile anche dal sito del Comitato di Basilea al seguente link: http://www.bis.org/bcbs/publ/comments/d319/overview.htm

- Banca d'Italia (2005), Il bilancio bancario: schemi e regole di compilazione, Circolare n.262 del 22 dicembre 2005 e successivi aggiornamenti.

- $\quad$ Banca d'Italia (2006), Nuove disposizioni di vigilanza prudenziale per le banche, Circolare n.263 del 27 dicembre 2006 e successivi aggiornamenti.

- Banca d'Italia (2008), Matrice dei Conti, Circolare 272 del 30 luglio 2008 e successivi aggiornamenti.

- Banca d'Italia (2012), Bollettino Economico n.70.

- Banca d'Italia (2013), Disposizioni di vigilanza prudenziale per le banche, Circolare n.285 del 17 dicembre 2013 e successivi aggiornamenti.

- Banca d'Italia (2018), Bollettino Economico n.1.

- Basel Committee on Banking Supervision (2004), Principles for Management and Supervision of Interest Rate Risk. Bank for International Settlements.

- Basel Committee on Banking Supervision (2006), International Convergence of Capital Measurement and Capital Standards: a Revised Framework. Bank for International Settlements

- Basel Committee on Banking Supervision (2009), Range of practices and issues in economic capital frameworks. Bank for International Settlements.

- Basel Committee on Banking Supervision (2016), Interest rate risk in the banking book. Bank for International Settlements.

- Committee of European Bankig Supervision (2006), Technical aspects of the management of interest rate risk arising from nontrading activities under the supervisory review process.

- Cocozza R, Curcio D., Gianfrancesco I. (2015), Non- Maturity Deposits and banks' exposure to the interest rate risk: issues arising from the Basel regulatory frame work, in Journal of Risk, 17(5), 1-36.

- Cerrone R., Cocozza R., Curcio D., Gianfrancesco I. (2017), Does prudential regulation contribute to effective measurement and management of interest rate risk? Evidence from Italian banks, in Journal of Financial Stability, 30, 126-138.

- Curcio D., Gianfrancesco I. (2011), La misurazione del rischio di tasso di interesse del portafoglio bancario nell'ambito di Basilea 2: quali le possibili criticità nella ricerca di nuove best practices? in Neswletter AIFIRM, Anno 6, Numero 1.

- $\quad$ European Central Bank (2017), ECB sensitivity analysis of IRRBB, stress test 2017.

- $\quad$ European Central Bank (2017), Sensitivity Analysis of IRBB - Stress test 2017 Final results, 9 October 2017.

- Engle R.F., Granger C.W.J. (1987), Co-integration and error correction: representation, estimation and testing, in Econometrica, 55(2), 251-276.

- $\quad$ Entrop, O., Memmel, C., Wilkens, M., and Zeisler, A. (2008), Analyzing the interest rate risk of banks using time series of accounting-based data: evidence from Germany, Deutsche Bundesbank Discussion Paper Series 2 No.1.

- $\quad$ Entrop, O., Wilkens, M., and Zeisler, A. (2009), Quantifying the Interest Rate Risk of Banks: Assumptions Do Matter, European Financial Management 15(5), 1001-1018.

- $\quad$ European Banking Authority (2015), Guidelines on the management of interest rate risk arising from non-trading activities, 22 May.

- European Banking Authority (2015), Guidelines on the management of interest rate risk arising from non-trading activities, 5 October.

- European Banking Authority (2017), Question ID: 2017,3121, Date of pubblication: 07/07/2017. Il documento è disponibile al seguente link: http://www.eba.europa.eu/single-rule-book-qa/-/qna/view/publicId/2017_3121 
- $\quad$ European Banking Authority (2017), Draft Guidelines on the management of interest rate risk arising from non-trading book activities, Consultation Paper, 31 October.

- Federal Deposit Insurance Corporation (1997), History of Eighties - Lessons for the Future. Il documento è disponibile al seguente link: http://www.fdic.gov/bank/historical/history

- Fiori, R., and Iannotti, S. (2007), Scenario Based Principal Component Value-at-Risk: An Application to Italian Bank's Interest Rate Risk Exposure, in Journal of Risk 9(3), 63-99.

- Gianfrancesco I., Giliberto C. (2014), La vischiosità dei depositi a vista durante la recente crisi finanziaria: implicazioni in una prospettiva di risk management, in Newsletter AIFIRM, Anno 9, Numero 3.

- Gianfrancesco I. (2016), L'esposizione al rischio di tasso di interesse del portafoglio bancario: quali implicazioni per le strategie di Asset \& Liability Management? in Newsletter AIFIRM, Anno 11, Numero 3-4.

- Hodrick R.J., Prescott E.C. (1997), Postwar US business cycles: an empirical investigation, in Journal of Money, Credit and Banking, 29(1), 1-16.

- Houpt, J. V., and Embersit, J. A. (1991), A Method for Evaluating Interest Rate Risk in U.S. Commercial Banks, in Federal Reserve Bulletin, 77(2), 625-637.

- $\quad$ Lopez J.A. (1999), Regulatory evaluation of value-at-risk models, in Journal of Risk (1), 37-64.

- $\quad$ Nelson C.R., Siegel A.F. (1987), Parsimonious modelling of yield curve, in Journal of Business, 69, 473-490.

- Parlamento Europeo e Consiglio dell'Unione Europea (2013), Direttiva 2013/36/UE del 26 giugno 2013 e successivi aggiornamenti e proposte di modifica.

- Parlamento Europeo e Consiglio dell'Unione Europea (2013), Regolamento (UE) 575/2013 del 26 giugno 2013 e successivi aggiornamenti e proposte di modifica.

- Sierra, G. E. (2009), Can an accounting-based duration model effectively measure interest rate sensitivity? Il paper è disponibile sul sito SSRN al seguente link: http://papers.ssrn.com/sol3/papers.cfm? abstract_id=1488884

- $\quad$ Sierra G. E., and Yeager T. J. (2004), What Does the Federal Reserve's Economic Value Model Tell Us About Interest Rate Risk at U.S. Community Banks? in The Federal Reserve Bank of St. Louis Review 86(6).

- Wright D. M., and Houpt J. V. (1996), An Analysis of Commercial Bank Exposure to Interest Rate Risk, in Federal Reserve Bulletin 82(2), 115-28. 


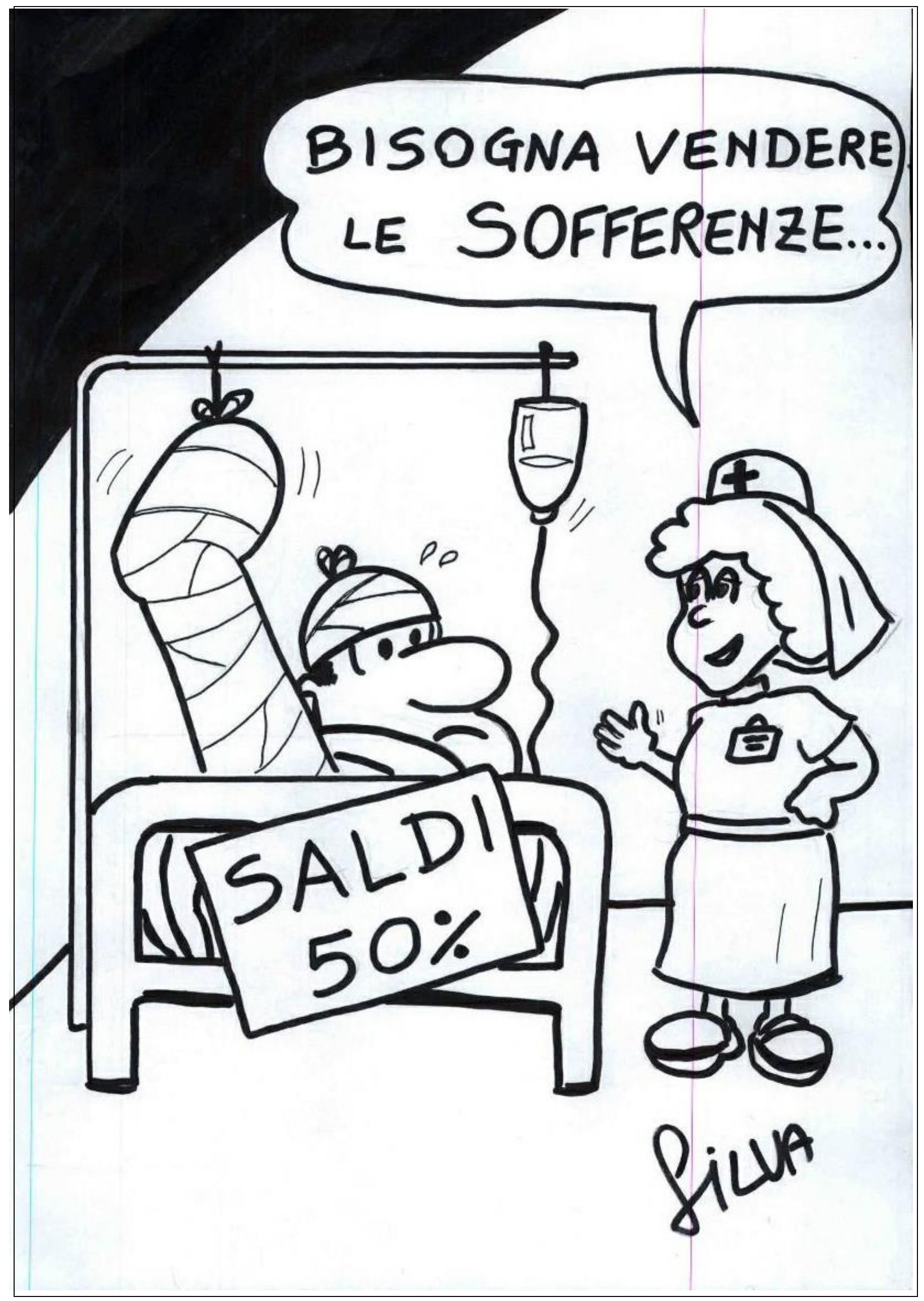




\section{RISK MANAGEMENT MAGAZINE}

Rivista dell'Associazione Italiana Financial Industry Risk Managers

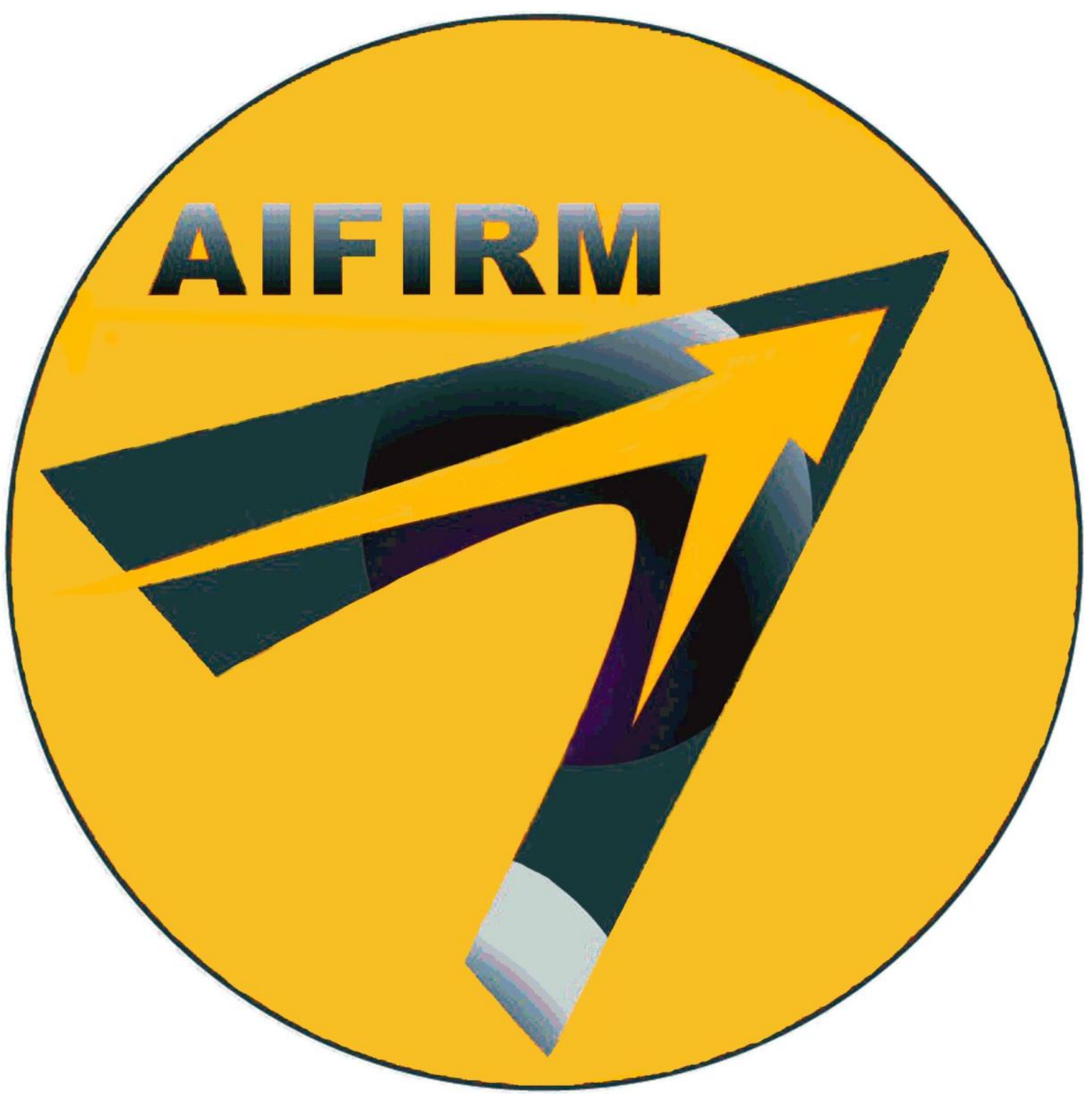

